Rev. Mat. IberoAmericAna 22 (2006), no. 3, 993-1067

\title{
Interpolated inequalities between exponential and Gaussian, Orlicz hypercontractivity and isoperimetry
}

\author{
Franck Barthe, Patrick Cattiaux and Cyril Roberto
}

\begin{abstract}
We introduce and study a notion of Orlicz hypercontractive semigroups. We analyze their relations with general $F$-Sobolev inequalities, thus extending Gross hypercontractivity theory. We provide criteria for these Sobolev type inequalities and for related properties. In particular, we implement in the context of probability measures the ideas of Maz'ja's capacity theory, and present equivalent forms relating the capacity of sets to their measure. Orlicz hypercontractivity efficiently describes the integrability improving properties of the Heat semigroup associated to the Boltzmann measures $\mu_{\alpha}(d x)=\left(Z_{\alpha}\right)^{-1} e^{-2|x|^{\alpha}} d x$, when $\alpha \in(1,2)$. As an application we derive accurate isoperimetric inequalities for their products. This completes earlier works by Bobkov-Houdré and Talagrand, and provides a scale of dimension free isoperimetric inequalities as well as comparison theorems.
\end{abstract}

\section{Introduction}

Sobolev type inequalities play an essential role in the study of the concentration phenomenon for probability measures. They are also a powerful tool to analyze the regularizing effects and the convergence to equilibrium of their associated symmetric semigroups. In particular, several surveys deal with the celebrated Poincaré (or spectral gap) inequality and the stronger logarithmic-Sobolev inequality and provide striking applications [32], [4], [3], [38], [33], [51].

2000 Mathematics Subject Classification: 26D10, 47D07 , 60E15, 60G10.

Keywords: Isoperimetry, Orlicz spaces, hypercontractivity, Boltzmann measure, Girsanov Transform, $F$-Sobolev inequalities. 
A concrete illustration can be given for the family of probability measures on the real line

$$
\mu_{\alpha}(d x)=\left(Z_{\alpha}\right)^{-1} e^{-2|x|^{\alpha}} d x, \quad \alpha>0 .
$$

These measures and their products $\mu_{\alpha}^{\otimes n}$ deserved particular attention in recent years, where the focus was on dimension free properties. They enter Talagrand's work on the concentration phenomenon for product measures. His study was continued by Ledoux [38], who strongly put forward the use of the logarithmic Sobolev inequality for concentration, and more recently by Bobkov and Houdré [17] who introduced $\mathbb{L}_{1}$-Sobolev type inequalities in order to study the more delicate isoperimetric problem. We review the main results concerning these measures and the associated semigroup $\left(\mathbf{P}_{t}^{\alpha}\right)_{t \geq 0}$ generated by the operator $\mathbf{L}_{\alpha}$ such that

$$
\mathbf{L}_{\alpha} f=\frac{1}{2} f^{\prime \prime}-\alpha|x|^{\alpha-1} \operatorname{sign}(x) f^{\prime} .
$$

For $\alpha>0$ the measures $\mu_{\alpha}$ verify a Weak Spectral Gap property introduced by Aida and Kusuoka as shown in [47]. They satisfy the Spectral Gap inequality exactly when $\alpha \geq 1$, and the logarithmic Sobolev inequality if and only if $\alpha \geq 2$.

When $\alpha<1$ there is no dimension free concentration, because this property requires exponential tails [52]. Oppositely, the measures enjoy very strong properties when $\alpha>2$. The corresponding semigroup is ultracontractive [35], meaning that for positive time it is continuous from $\mathbb{L}^{2}\left(\mu_{\alpha}\right)$ to $\mathbb{L}^{\infty}$. The measures satisfy a dimension free Gaussian isoperimetric inequality [8, Theorem 9], and this is as bad as it gets by the Central Limit Theorem. Recently, Bobkov and Zegarlinski [19] obtained concentration inequalities for these measures but for the $\ell_{\alpha}^{n}$-distance on $\mathbb{R}^{n}$. Their results are based on appropriate modification of the logarithmic Sobolev inequality, and show different behaviors for different values of $\alpha$. This was not the case when considering the Euclidean distance.

The range $\alpha \in[1,2]$ presents very interesting properties. We start with the Gaussian case, $\alpha=2$, which is best understood. Concentration of measure and isoperimetry in Gauss space are now classical (see e.g. [38, 7]). It is remarkable that they are both dimension free. Recall that the isoperimetric inequality asserts in particular that for $A \subset \mathbb{R}^{n}$ with $\mu_{2}^{\otimes n}(A)=\mu_{2}((-\infty, t])$ one has for all $h>0$,

$$
\mu_{2}^{\otimes n}\left(A+h B_{2}^{n}\right) \geq \mu_{2}((-\infty, t+h]) .
$$

Here $B_{2}^{n}$ is the $n$-dimensional Euclidean ball. Taking limits one obtains that among sets of given Gaussian measure, half-spaces have minimal Gaussian boundary measure. 
On the other hand, the Gaussian measure has remarkable analytic properties: the corresponding Ornstein-Uhlenbeck semigroup is hypercontractive, as discovered by Nelson [45]. Gross proved that this fact is equivalent to the logarithmic Sobolev inequality [31]. Let us also mention that the Gaussian measure is the prototype of strictly log-concave measures. It was a success of the Bakry-Emery formalism to allow the extension of most of the previous results to abstract semigroups with positive curvature (see [6] for logarithmic Sobolev inequalities and [7] for Gaussian isoperimetry and an abstract version of the Levy-Gromov theorem).

The two sided exponential measure $\alpha=1$ is also well understood. Talagrand's paper [52] provides the following very precise estimate: if $A \subset \mathbb{R}^{n}$ verifies $\mu_{1}^{\otimes n}(A)=\mu_{1}((-\infty, t])$ then for all $h \geq 0$ it holds

$$
\mu_{1}^{\otimes n}\left(A+h B_{1}^{n}+\sqrt{h} B_{2}^{n}\right) \geq \mu_{1}((-\infty, t+h / K]),
$$

where $K$ is a universal constant and $B_{p}^{n}=\left\{x \in \mathbb{R}^{n} ; \sum_{i=1}^{n}\left|x_{i}\right|^{p} \leq 1\right\}$. See also $[41,55]$. In a slightly weaker form, such a statement was recovered by Bobkov and Ledoux [18], via a modified logarithmic Sobolev inequality which is equivalent to Poincaré inequality. Thus products of measures on $\mathbb{R}^{d}$ with a spectral gap satisfy a concentration inequality on the exponential model. Moreover, Bobkov and Houdré [16] proved that $\mu_{1}^{\otimes n}$ satisfies a dimension free isoperimetric inequality of Cheeger. The proof relies on an $\mathbb{L}_{1}$ version of the Poincaré inequality, and the statement can be rephrased as follows: let $A \subset \mathbb{R}^{n}$ with $\mu_{1}^{\otimes n}(A)=\mu_{1}((-\infty, t])$ then for all $h \geq 0$

$$
\mu_{1}^{\otimes n}\left(A+h B_{2}^{n}\right) \geq \mu_{1}\left(\left(-\infty, t+\frac{h}{2 \sqrt{6}}\right]\right) .
$$

This result completes the one of Talagrand. It is weaker for large values of $h$ but gives isoperimetric information as $h$ goes to zero.

This paper provides a precise description of concentration and isoperimetry for product of distributions which are intermediate between the exponential and the Gaussian laws. This range is particularly relevant because it contains all models of dimension free concentration and isoperimetric inequality, of high probabilistic importance. Indeed, as we briefly mentioned, dimension free concentration requires exponential or faster tails, and cannot be faster than Gaussian by the Central Limit Theorem.

Going back to our examples, let us present what is known for $\mu_{\alpha}$ when $\alpha \in(1,2)$. The concentration phenomenon is already well described. Indeed, Talagrand's exponential inequality transfers to $\mu_{\alpha}$ for any $\alpha>1$ [53] and ensures that

$$
\mu_{\alpha}^{\otimes n}\left(A+h^{1 / \alpha} B_{\alpha}^{n}+\sqrt{h} B_{2}^{n}\right) \geq 1-\frac{1}{\mu_{\alpha}^{\otimes n}(A)} e^{-h / K}
$$

for every $A \subset \mathbb{R}^{n}$ and $h>0$. In particular if $\alpha \in(1,2)$ and $\mu_{\alpha}^{n}(A) \geq 1 / 2$ one gets that for $h \geq 1, \mu_{\alpha}^{n}\left(A+h B_{2}^{n}\right) \geq 1-2 e^{-h^{\alpha} / K}$. 
A functional approach to this fact was recently discovered by Latała and Oleszkiewicz [36]. These authors established the following family of Sobolev inequalities: there exists a universal constant $C$ such that for all $1<p<2$ it holds

$$
\int f^{2} d \mu_{\alpha}-\left(\int|f|^{p} d \mu_{\alpha}\right)^{\frac{2}{p}} \leq C(2-p)^{2\left(1-\frac{1}{\alpha}\right)} \int\left(f^{\prime}\right)^{2} d \mu_{\alpha}
$$

for smooth enough $f$. For $\alpha=2$ these inequalities are due to Beckner [13]. Inequalities (1.3) interpolate between Poincaré and log-Sobolev. They enjoy the tensorisation property and imply dimension free concentration with decay $e^{-K t^{\alpha}}$ as expected. Obviously [36] was the starting point of an extension of the log-Sobolev approach to concentration, encompassing more general behaviors. Recently two of us simplified the proof of (1.3) and characterized all measures on $\mathbb{R}$ satisfying the same property [12] (such a criterion for log-Sobolev already existed, thanks to Bobkov and Götze [15]). See [20] for other developments. Inequalities (1.3) above are part of a more general family denoted $\Phi$-Sobolev inequalities. A study of this family in connection with some aspects of semi-group theory is done in [25].

The initial goal of this work is to obtain a precise dimension free isoperimetric inequality for $\mu_{\alpha}^{\otimes n}$ when $\alpha \in(1,2)$. Namely we want to prove that there exists a constant $C$ such that for all $n \in \mathbb{N}$

$$
\left(\mu_{\alpha}^{\otimes n}\right)_{s}(\partial A) \geq C \mu_{\alpha}^{\otimes n}(A)\left(\log \left(\frac{1}{\mu_{\alpha}^{\otimes n}(A)}\right)\right)^{1-\frac{1}{\alpha}},
$$

for all $A$ such that $\mu_{\alpha}^{\otimes n}(A) \leq \frac{1}{2}$, where $\mu_{s}(\partial A)$ denotes the surface measure of $A$ (see section 8). This bound is known for $\alpha=1$ [16] and $\alpha=2$ [7] and can be deduced from [14] in dimension 1. So (1.4) is exactly what is expected. This result is stronger than the concentration result. Indeed it implies that for all $n$ and $A \subset \mathbb{R}^{n}$ with $\mu_{\alpha}^{\otimes n}(A)=\mu_{\alpha}((-\infty, t])$ one has

$$
\mu_{\alpha}^{\otimes n}\left(A+h B_{2}^{n}\right) \geq \mu_{\alpha}((-\infty, t+h / K]) .
$$

This interpolates between (1.2) and (1.1).

Inequality (1.4) will be shown in Theorem 46 as the achievement of a somewhat intricate story. Actually, we prove much more and develop several useful methods on the way. They should find a field of applications in the study of empirical processes or in statistical physics.

Before describing the organization of the paper, let us explain that our proof relies on a method initiated by Ledoux [37] and improved in [7]. It can be summarized as follows: any integrability improving property of a semigroup with curvature bounded from below provides isoperimetric information for the invariant measure. Hence our problem translates to a 
question on the semigroup $\left(\mathbf{P}_{t}^{\alpha}\right)_{t \geq 0}$ for $\alpha \in(1,2)$. However, a theorem of Høegh-Krohn and Simon [34] shows that $\mathbf{P}_{t}^{\alpha}$ is never continuous from $\mathbb{L}^{2}\left(\mu_{\alpha}\right)$ to $\mathbb{L}^{2+\varepsilon}\left(\mu_{\alpha}\right)$. Since the $\mathbb{L}^{p}$ scale is too rough for our problem, we analyze the regularizing properties in appropriate scales of Orlicz spaces and ask whether the semigroup maps $\mathbb{L}^{2}\left(\mu_{\alpha}\right)$ into a smaller Orlicz space.

Section 2 contains the required elements on Orlicz spaces.

Section 3 presents a sufficient condition on the Young function $\tau$ for $\mathbf{Q}_{t}^{\alpha}$ (a slightly modified $\mathbf{P}_{t}^{\alpha}$ ) to map continuously $\mathbb{L}^{2}$ into $\mathbb{L}_{\tau}$, for a fixed $t$. This condition relies on the probabilistic representation of $\mathbf{P}_{t}^{\alpha}$ (Girsanov transformation) and on martingale methods inspired by [35, 21]. Unfortunately the method cannot reach the contraction property (only boundedness, simply called $\tau$-Orlicz hyperboundedness) and does not easily yield explicit bounds. The criterion readily extends to certain perturbations of an ultracontractive semi-group.

In order to get contraction results and explicit bounds, we build in Section 4 the full analogue of Gross theory. Following [22] we start with the analogue of a result by Høegh-Krohn and Simon (Theorem 4): if $\mathbf{P}_{t_{0}}$ is continuous from $\mathbb{L}^{2}(\mu)$ into $\mathbb{L}_{\tau}(\mu)$ then $\mu$ satisfies a defective logarithmic Orlicz-Sobolev inequality. This is actually a particular F-Sobolev inequality as studied in $[57,30]$ (the notion apparently goes back to Concordet). For the $|x|^{\alpha}$ Boltzmann measure $\mu_{\alpha}$, it is equivalent (see (4.8)) to the following result of Rosen [49]: there exist $A$ and $B$ such that for $\int f^{2} e^{-2|y|^{\alpha}} d y=1$,

$$
\int f^{2}(y)\left(\log _{+}(|f(y)|)\right)^{2\left(1-\frac{1}{\alpha}\right)} e^{-2|y|^{\alpha}} d y \leq A \int|\nabla f|^{2} e^{-2|y|^{\alpha}} d y+B
$$

See Adams [1] for extensions and Zegarlinski [59] for an application of Rosen type inequalities to the study for Gibbs measures with non-Gaussian tails.

Next we consider homogeneous $F$-Sobolev inequalities. One of our main results is Theorem 6 where we obtain the equivalence between a $F$-Sobolev inequality and the $\tau_{q}$-Orlicz-hyperboundedness (or hypercontractivity) of the whole semigroup for $\tau_{q}(x):=x^{p} e^{q F\left(x^{p}\right)}$. Under a few assumptions on $F$, the time evolution of the regularizing effect is quantified. A weak form of part of these results appeared in [30, Theorem 1.2 and Theorem 2.4]. These authors proved that a particular tight $F$-Sobolev inequality is equivalent to Orlicz-hyperboundedness for some time. Their motivation was a criterion for the generator to have a non-empty essential spectrum (see [30, 57] for connections with super-Poincaré inequalities). By Theorem 6 , a tight $F$ Sobolev inequality for a nonnegative $F$ guarantees that the semigroup is Orlicz-hypercontractive. We conclude this section by extending the well known inequality of Rothaus [50]: under spectral gap assumption this allows to turn certain defective $F$-Sobolev inequalities into tight ones. 
Section 5 provides a thorough study of Sobolev type inequalities. In the Gaussian context the log-Sobolev inequality is canonical and has plenty of remarkable properties: it tensorizes, provides concentration via Herbst argument, hypercontractivity and entropy decay along the semigroup. In our more general setting, in particular for $\mu_{\alpha}, \alpha \in(1,2)$, no such miracle happens. Several Sobolev inequalities are available. However none of them concentrates all good properties. This is why we undertake a precise study of Beckner type inequalities, of homogeneous $F$-Sobolev inequalities and additive $\varphi$-Sobolev inequalities also called $\Phi$-Entropy inequalities (wee shall not discuss the latter in terms of exponential decay of $\Phi$-entropy. See $[25,58])$. Our strategy is to provide each inequality with a simpler reduced form relating the measure of sets to their $\mu$-capacity. This notion was alluded to by the first and last-named authors in [12]. Here we use it systematically in the spirit of Maz'ja [42]. Note that the probabilistic setting is delicate since constant functions are equality cases in all our inequalities. Our approach is an extension to any dimension of the criteria on the real line recently obtained through Hardy inequalities $[15,12]$. It provides new criteria and equivalences between several Sobolev inequalities. A final figure summarizes the situation.

Section 6 deals with the consequences of generalized Beckner inequalities for the concentration of measure. They are immediate from the method of Latała-Oleszkiewicz, and where discussed independently by Wang [56]. Our contribution here comes from our sharp criteria for these inequalities. In particular we give general neat conditions for products of measures on $\mathbb{R}$ to enjoy dimension free concentration with rate $e^{-\Phi(t)}$ where $\Phi(t)$ is convex, but less than $t^{2}$. Under reasonable assumptions the criterion is satisfied by the measure $e^{-\Phi(t)} / Z$ itself, so the concentration is sharp. For other results in connection with mass transportation, see also [58, 23, 29].

Section 7 illustrates all the previous results in the case of $|x|^{\alpha}$ Boltzmann measures. In this concrete situation we explain how to deal with the technical conditions involved. We also develop a perturbation argument similar to the one of [21, section 4].

The final section deduces isoperimetric inequalities from semigroup hyperboundedness properties. The claimed infinite dimensional isoperimetric bound (1.4) is derived. As a consequence a family of comparison theorems is provided.

For sake of clarity we decided not to develop our argument in its full generality. However, most of our results easily extend to more general situations, encompassing diffusion operators on Riemannian manifolds. This is the case of the Gross-Orlicz theory, of the reductions to inequalities between capacity and measure. The final isoperimetric lower bounds would work when the curvatures of the generators is bounded from below. 


\section{Orlicz hypercontractivity}

In this section we discuss a weakened form of hypercontractivity and hyperboundedness, replacing $\mathbb{L}^{p}$ spaces by Orlicz spaces. We start with recalling basic notions about these spaces. Some definitions are not the usual ones used e.g. in the book by Rao and Ren [46].

In the sequel we consider a complementary pair $\left(\tau^{*}, \tau\right)$ of continuous and even Young's functions (i.e. $\tau^{*}$ is the Fenchel-Legendre dual function of $\tau$, both being convex functions vanishing at the origin) satisfying

$$
\lim _{y \rightarrow+\infty} \frac{\tau(y)}{y^{2}}=+\infty \quad \text { and } \quad \lim _{y \rightarrow+\infty} \frac{\tau(y)}{y^{p}}=0 \quad \text { for } p>2 .
$$

It follows that

$$
\lim _{y \rightarrow+\infty} \frac{\tau^{*}(y)}{y^{2}}=0 \quad \text { and } \quad \lim _{y \rightarrow+\infty} \frac{\tau^{*}(y)}{y^{p}}=+\infty \quad \text { for } p<2 .
$$

We assume that $\tau$ and $\tau^{*}$ both satisfy the $\Delta_{2}$ condition (i.e. $\tau(2 y) \leq K \tau(y)$ for some $K>1$ and $y \geq y_{1} \geq 0$, and a similar result for $\tau^{*}$ with possibly different $K^{*}$ and $y_{1}^{*}$ ). It follows that they both satisfy the $\nabla_{2}$ condition too (i.e. $2 l \tau(y) \leq \tau(l y)$ for some $l>1$ and $y \geq \overline{y_{1}} \geq 0$ and similarly for $\tau^{*}$ with $l^{*}$ and $\left.\bar{y}_{1}^{*}\right)$, see $[46$, p. 22, 23].

We also assume that the pair $\left(\tau, \tau^{*}\right)$ satisfies $\tau(0)=\tau^{*}(0)=0$. The space $\mathbb{L}_{\tau}(\mu)$ is the space of measurable functions $f$ such that

$$
I_{\tau}(f) \stackrel{\text { def }}{=} \int \tau(|f|) d \mu<+\infty .
$$

Thanks to the $\Delta_{2}$ property, $\mathbb{L}_{\tau}$ and $\mathbb{L}_{\tau^{*}}$ are linear spaces. We shall use two norms on each space,

$$
\begin{aligned}
& N_{\tau}(f) \stackrel{\text { def }}{=} \inf \left\{u>0 ; I_{\tau}\left(\frac{f}{u}\right) \leq \tau(1)\right\}, \\
& \|f\|_{\tau} \stackrel{\text { def }}{=} \sup \left\{\int|f g| d \mu ; N_{\tau^{*}}(g) \leq 1\right\},
\end{aligned}
$$

with similar definitions for $\tau^{*}$. The first one is called the gauge or Luxemburg norm.

Note that $N_{\tau}$ is unchanged when $\tau$ is multiplied by a positive constant. Hence if there exists $\alpha>0$ such that $\alpha \tau(1)+\alpha \tau^{*}(1 / \alpha)=1$, we may consider the pair $\left(\eta, \eta^{*}\right)$ where $\eta=\alpha \tau$. It is normalized in the sense that $\eta(1)+\eta^{*}(1)=1$. In particular if $\tau^{\prime}(1)$ exists and is positive, we may take $\eta=\tau / \tau^{\prime}(1)$. However, in general, this normalization is not assumed. 
With our definitions, and thanks to the regularity properties of $\tau$ and $\tau^{*}$ it is known that for $f \in \mathbb{L}_{\tau}$ and $g \in \mathbb{L}_{\tau^{*}}$ it holds (see [46, Proposition 1, p. 58])

$$
I_{\tau}\left(\frac{f}{N_{\tau}(f)}\right)=\tau(1) \quad \text { and } \quad N_{\tau}(\mathbb{I})=\|\mathbb{I}\|_{\tau}=1
$$

The analogue of Hölder's inequality is $\int|f g| d \mu \leq\left(\tau(1)+\tau^{*}(1)\right) N_{\tau}(f) N_{\tau^{*}}(g)$. It implies that

$$
\|f\|_{\tau} \leq\left(\tau(1)+\tau^{*}(1)\right) N_{\tau}(f) .
$$

The $\Delta_{2}$ condition ensures that $\left(\mathbb{L}_{\tau}, N_{\tau}\right)$ is a reflexive Banach space with dual space $\left(\mathbb{L}_{\tau^{*}},\|\cdot\|_{\tau^{*}}\right)$ [46, Theorem 6 , p. 105]. Also note that $N_{\tau}$ and $\|\cdot\|_{\tau}$ are equivalent (see [46, (18), p. 62$]$ ) and that the subset of bounded functions is dense in $\mathbb{L}_{\tau}$. The same holds when we replace $\tau$ by $\tau^{*}$.

Finally remark that if $N_{\tau}(f) \geq 1$,

$$
\tau(1)=I_{\tau}\left(\frac{f}{N_{\tau}(f)}\right) \leq \frac{1}{N_{\tau}(f)} I_{\tau}(f)
$$

so that

$$
N_{\tau}(f) \leq \max \left(1, \frac{I_{\tau}(f)}{\tau(1)}\right)
$$

Conversely if $f(x) \geq N_{\tau}(f) y_{1}$ (recall the definition of $\Delta_{2}$ ) then

$$
\tau(f(x))=\tau\left(N_{\tau}(f) \frac{f(x)}{N_{\tau}(f)}\right) \leq K^{\frac{\log \left(N_{\tau}(f)\right)}{\log (2)}+1} \tau\left(\frac{f(x)}{N_{\tau}(f)}\right) .
$$

It follows that

$$
I_{\tau}(f) \leq \tau\left(N_{\tau}(f) y_{1}\right)+K^{\frac{\log \left(N_{\tau}(f)\right)}{\log (2)}+1} \tau(1) .
$$

Remark 1. Our definitions of norms on $\mathbb{L}_{\tau}$ are not standard. Our choice ensures that the constant function $\mathbb{I}$ has norm one in all spaces. If we replace $\tau(1)$ by 1 in the definition of $N_{\tau}$ we obtain the usual gauge norm $N_{\tau}^{1}$. It is equivalent to $N_{\tau}$. Indeed (2.6) implies

$$
N_{\tau}(f) \leq \max \left(1, \frac{1}{\tau(1)}\right) N_{\tau}^{1}(f)
$$

and a similar argument yields $N_{\tau}^{1}(f) \leq \max (1, \tau(1)) N_{\tau}(f)$. Our definition of $\|\cdot\|_{\tau}$ does not coincide with the usual Orlicz norm [46, Definition 2, p. 58], but it is natural by duality. 
We are ready to state our main definitions.

Definition 1 (Orlicz-hyperboundedness). We say that a $\mu$-symmetric semigroup $\left(\mathbf{P}_{t}\right)_{t \geq 0}$ is Orlicz-hyperbounded if there exist $t>0$, and a Young function $\tau$ with

$$
\lim _{y \rightarrow+\infty} \frac{\tau(y)}{y^{2}}=+\infty \quad \text { and } \quad \lim _{y \rightarrow+\infty} \frac{\tau(y)}{y^{p}}=0 \quad \text { for } p>2,
$$

such that $\mathbf{P}_{t}$ is a continuous mapping from $\mathbb{L}^{2}(\mu)$ into $\mathbb{L}_{\tau}(\mu)$.

Definition 2 (Orlicz-hypercontractivity). We say that a $\mu$-symmetric semigroup $\left(\mathbf{P}_{t}\right)_{t>0}$ is Orlicz-hypercontractive if there exist $t>0$ and a Young function $\tau$ as in Definition 1 such that $\mathbf{P}_{t}$ is a contraction from $\mathbb{L}^{2}(\mu)$ into $\left(\mathbb{L}_{\tau}(\mu), N_{\tau}\right)$. Equivalently $\mathbf{P}_{t}$ is a contraction from $\left(\mathbb{L}_{\tau^{*}}(\mu),\|\cdot\|_{\tau^{*}}\right)$ into $\mathbb{L}^{2}(\mu)$.

Thanks to Jensen's inequality, for all $s>0, \mathbf{P}_{s}$ is a contraction in both $\left(\mathbb{L}_{\tau}(\mu), N_{\tau}\right)$ and $\left(\mathbb{L}_{\tau^{*}}(\mu),\|\cdot\|_{\tau^{*}}\right)$ with norm 1 , achieved by constant functions. In particular if the contraction property in Definition 2 holds for $t$, it holds for all $s>t$.

The next section gives a criterion for a semi-group to be Orlicz-hyperbounded.

\section{Orlicz hyperboundedness for $|x|^{\alpha}$ and general Boltz- mann measures}

First we present our argument for the potential $|x|^{\alpha}, \alpha \in(1,2)$. Actually the method requires $\mathcal{C}^{2}$ regularity so we prefer to work with a well behaved modification of the latter. For example we may consider the function $u_{\alpha}$ defined on $\mathbb{R}$ by

$$
u_{\alpha}(x)= \begin{cases}|x|^{\alpha} & \text { for }|x|>1 \\ \frac{\alpha(\alpha-2)}{8} x^{4}+\frac{\alpha(4-\alpha)}{4} x^{2}+\left(1-\frac{3}{4} \alpha+\frac{1}{8} \alpha^{2}\right) & \text { for }|x| \leq 1\end{cases}
$$

It is easy to see that $u_{\alpha}$ is $\mathcal{C}^{2}$, convex and bounded below by $1-\frac{3}{4} \alpha+\frac{1}{8} \alpha^{2}>0$. One can also check that $|x|^{\alpha} \leq u_{\alpha}(x) \leq \max \left(1,|x|^{\alpha}\right)$, for all $x \in \mathbb{R}$. The associated Boltzmann probability measure on $\mathbb{R}^{n}$ is defined as

$$
\nu_{\alpha}^{\otimes n}(d x)=Z_{\alpha}^{-n} e^{-2 \sum_{i=1}^{n} u_{\alpha}\left(x_{i}\right)} d x=Z_{\alpha}^{-n} e^{-2 U_{\alpha, n}(x)} d x
$$

where $Z_{\alpha}$ is the proper normalizing constant and $U_{\alpha, n}(x) \stackrel{\text { def }}{=} \sum_{i=1}^{n} u_{\alpha}\left(x_{i}\right)$. For notational convenience we shall write $U_{\alpha}$ for $U_{\alpha, n}$. Similarly the forthcoming objects depend on the dimension $n$. This dependence will be recalled through the notation $\nu_{\alpha}^{\otimes n}$ only. 
To the Boltzmann measure is associated a symmetric semi-group $\left(\mathbf{P}_{t}^{\alpha}\right)_{t \geq 0}$ generated by the operator

$$
A_{\alpha}=\frac{1}{2} \Delta-\nabla U_{\alpha} \cdot \nabla
$$

One can show (see [51], [22, Section 7] or [21]) that the semi-group is given by

$$
\left(\mathbf{P}_{t}^{\alpha} h\right)(x)=e^{U_{\alpha}(x)} \mathbb{E}^{\mathbb{P} x}\left[h\left(X_{t}\right) e^{-U_{\alpha}\left(X_{t}\right)} M_{t}\right]
$$

where $\mathbb{P}_{x}$ is the Wiener measure such that $\mathbb{P}_{x}\left(X_{0}=x\right)=1$ (i.e. under $\mathbb{P}_{x}$, $X$. is a $n$-dimensional Brownian motion starting from $x)$ and $M_{t}$ is defined as

$$
M_{t}=\exp \left(\frac{1}{2} \int_{0}^{t}\left(\Delta U_{\alpha}-\left|\nabla U_{\alpha}\right|^{2}\right)\left(X_{s}\right) d s\right) .
$$

In this section, we have chosen the probabilistic normalization of the Laplacian $\frac{1}{2} \Delta$ in order to avoid extra variance on the Brownian motion.

Since $e^{U_{\alpha}}$ belongs to all $\mathbb{L}^{p}\left(\nu_{\alpha}^{\otimes n}\right)$ for $p<2$, an almost necessary condition for Orlicz hypercontractivity is that $\mathbf{P}_{t}^{\alpha}\left(e^{U_{\alpha}}\right)$ belongs to some $\mathbb{L}_{\tau}\left(\nu_{\alpha}^{\otimes n}\right)$. In [21, Section 3], the "Well Method" of Kavian, Kerkyacharian and Roynette [35] is pushed further and allows to estimate $\mathbf{P}_{t}^{\alpha}\left(e^{U_{\alpha}}\right)$ when $\alpha \geq 2$. In the following, we extend this method to the case $1<\alpha<2$.

Theorem 1. Let $\alpha \in(1,2)$ and $n \geq 1$. Let $\tau$ be a Young function satisfying $\tau(y)=y^{2} \psi(y)$ for some positive and non decreasing function $\psi$ going to $+\infty$ at infinity. Assume that $\psi$ satisfies the $\Delta_{2}$ condition: there exists constants $k, y_{1}$ such that $\psi(2 y) \leq k \psi(y)$ provided $y>y_{1}$. Let $\nu_{\alpha}^{\otimes n}$ be the Boltzmann measure defined on $\mathbb{R}^{n}$ in (3.2).

Then $\mathbf{P}_{t}^{\alpha}\left(e^{U_{\alpha}}\right)$ belongs to $\mathbb{L}_{\tau}\left(\nu_{\alpha}^{\otimes n}\right)$ if there exists a constant $C<\alpha^{2}$ such that

$$
\int_{\mathbb{R}^{n}} \psi\left(e^{U_{\alpha}(x)}\right) e^{-C t U_{\alpha}(x)^{2-2 / \alpha}} d x<+\infty .
$$

The proof below can be used to get explicit bounds, depending on $n$. Proof. First remark that $U_{\alpha}$ satisfies

$$
\frac{1}{2}\left(\left|\nabla U_{\alpha}\right|^{2}(x)-\Delta U_{\alpha}(x)\right) \geq G_{\alpha}\left(U_{\alpha}(x)\right)-c_{\alpha}=H_{\alpha}\left(U_{\alpha}(x)\right),
$$

with $G_{\alpha}(y)=\frac{\alpha^{2}}{2}|y|^{2\left(1-\frac{1}{\alpha}\right)}$ and $c_{\alpha}=n\left(1+\frac{1}{2} \alpha(\alpha-1)\right)$, with our choice of $u_{\alpha}$ for $|x| \leq 1$. Since $G_{\alpha}$ is subbadditive, it is enough to prove the above inequality in dimension $n=1$. We leave the details to the reader. Note that $H_{\alpha}$ admits an inverse $H_{\alpha}^{-1}$ defined on $\left[-c_{\alpha} ;+\infty\right)$ with values in $\mathbb{R}^{+}$. 
For $\varepsilon>0$ define the stopping time $T_{x}$ as

$$
T_{x}=\inf \left\{s>0 ; \frac{1}{2}\left(\left|\nabla U_{\alpha}\right|^{2}-\Delta U_{\alpha}\right)\left(X_{s}\right) \leq H_{\alpha}\left(U_{\alpha}(x)-\varepsilon\right)\right\} .
$$

Note that for all $x \in \mathbb{R}^{n}, T_{x}>0 \mathbb{P}_{x}$ a.s. provided $U_{\alpha}(x)-\varepsilon \geq 0$ and that on $T_{x}<+\infty$

$$
U_{\alpha}\left(X_{T_{x}}\right) \leq H_{\alpha}^{-1}\left(\frac{1}{2}\left(\left|\nabla U_{\alpha}\right|^{2}-\Delta U_{\alpha}\right)\left(X_{T_{x}}\right)\right) \leq U_{\alpha}(x)-\varepsilon .
$$

Introducing the previous stopping time we get

$$
\mathbb{E}^{\mathbb{P} x}\left[M_{t}\right]=\mathbb{E}^{\mathbb{P}_{x}}\left[M_{t} \mathbb{I}_{t<T_{x}}\right]+\mathbb{E}^{\mathbb{P} x}\left[M_{t} \mathbb{I}_{T_{x} \leq t}\right]=A+B,
$$

with

$$
A=\mathbb{E}^{\mathbb{P}_{x}}\left[M_{t} \mathbb{I}_{t<T_{x}}\right] \leq \exp \left(-t H_{\alpha}\left(U_{\alpha}(x)-\varepsilon\right)\right),
$$

and $\mathbb{E}^{\mathbb{P}_{x}}\left[M_{t} \mathbb{I}_{T_{x} \leq t}\right]$. In order to bound $B$ from above we introduce the nonpositive quantity $\frac{1}{2}\left(\Delta U_{\alpha}-\left|\nabla U_{\alpha}\right|^{2}\right)-c_{\alpha}$

$$
\begin{aligned}
B & =\mathbb{E}^{\mathbb{P}_{x}}\left[M_{t} \mathbb{I}_{T_{x} \leq t}\right] \\
& \leq e^{c_{\alpha} t} \mathbb{E}^{\mathbb{P}_{x}}\left[\exp \left(\int_{0}^{t}\left(\frac{1}{2}\left(\Delta U_{\alpha}-\left|\nabla U_{\alpha}\right|^{2}\right)-c_{\alpha}\right)\left(X_{s}\right) d s\right) \mathbb{I}_{T_{x} \leq t}\right] \\
& \leq e^{c_{\alpha} t} \mathbb{E}^{\mathbb{P}_{x}}\left[\exp \left(\int_{0}^{T_{x}}\left(\frac{1}{2}\left(\Delta U_{\alpha}-\left|\nabla U_{\alpha}\right|^{2}\right)-c_{\alpha}\right)\left(X_{s}\right) d s\right) \mathbb{I}_{T_{x} \leq t}\right] \\
& \leq e^{c_{\alpha} t} \mathbb{E}^{\mathbb{P}_{x}}\left[\exp \left(\int_{0}^{T_{x}}\left(\frac{1}{2}\left(\Delta U_{\alpha}-\left|\nabla U_{\alpha}\right|^{2}\right)\right)\left(X_{s}\right) d s\right) \mathbb{I}_{T_{x} \leq t}\right] \\
& =e^{c_{\alpha} t} \mathbb{E}^{\mathbb{P}_{x}}\left[M_{T_{x}} \mathbb{I}_{T_{x} \leq t}\right] .
\end{aligned}
$$

Note that $N_{s}:=e^{-U_{\alpha}\left(X_{s}\right)} M_{s}$ is a bounded $\mathbb{P}_{x}$ martingale (this can be seen by the Itô calculus). Hence, Doob Optional Stopping Theorem ensures that $\mathbb{E}^{\mathbb{P} x} N_{t \wedge T_{x}}=\mathbb{E} N_{0}=e^{-U_{\alpha}(x)}$. Thus

$$
\mathbb{E}^{\mathbb{P} x}\left[e^{-U_{\alpha}\left(X_{T_{x}}\right)} M_{T_{x}} \mathbb{I}_{T_{x} \leq t}\right] \leq \mathbb{E}^{\mathbb{P}_{x}}\left[e^{-U_{\alpha}\left(X_{t \wedge T_{x}}\right)} M_{t \wedge T_{x}}\right]=e^{-U_{\alpha}(x)} .
$$

According to (3.7), $e^{-U_{\alpha}\left(X_{T_{x}}\right)} \geq e^{\varepsilon} e^{-U_{\alpha}(x)}$, so that thanks to (3.10),

$$
\mathbb{E}^{\mathbb{P} x}\left[M_{T_{x}} \mathbb{I}_{T_{x} \leq t}\right] \leq e^{-\varepsilon} .
$$

Combining this with (3.9) and using (3.8) we obtain

$$
\mathbb{E}^{\mathbb{P} x}\left[M_{t}\right] \leq e^{-t H_{\alpha}\left(U_{\alpha}(x)-\varepsilon\right)}+e^{-\varepsilon} e^{c_{\alpha} t} .
$$


Choosing $\varepsilon=\beta U_{\alpha}(x)$ for some $\beta<1$ the latter inequality becomes

$$
\mathbb{E}^{\mathbb{P}_{x}}\left[M_{t}\right] \leq e^{c_{\alpha} t}\left(e^{-\frac{t}{2} \alpha^{2}\left((1-\beta) U_{\alpha}(x)\right)^{2-\frac{2}{\alpha}}}+e^{-\beta U_{\alpha}(x)}\right),
$$

where for $|x|$ large, the dominating term is the first one since $\alpha<2$. Also note that (3.5) yields $M_{t} \leq e^{c_{\alpha} t}$ and the rough bound $\mathbb{E}^{\mathbb{P}_{x}}\left[M_{t}\right] \leq e^{c_{\alpha} t}$.

Finally $\mathbf{P}_{t}^{\alpha}\left(e^{U_{\alpha}}\right)=e^{U_{\alpha}} \mathbb{E}^{\mathbb{P}_{x}}\left[M_{t}\right]$ belongs to $\mathbb{L}_{\tau}\left(\nu_{\alpha}^{\otimes n}\right)$ provided

$$
\int_{\mathbb{R}^{n}} \mathbf{P}_{t}^{\alpha}\left(e^{U_{\alpha}}\right)^{2} \psi\left(\mathbf{P}_{t}^{\alpha}\left(e^{U_{\alpha}}\right)\right) d \nu_{\alpha}^{\otimes n}<+\infty
$$

This is equivalent to

$$
\int_{\mathbb{R}^{n}} \mathbb{E}^{\mathbb{P}_{x}}\left[M_{t}\right]^{2} \psi\left(e^{U_{\alpha}(x)} \mathbb{E}^{\mathbb{P}_{x}}\left[M_{t}\right]\right) d x<+\infty .
$$

Using the above bounds on $\mathbb{E}^{\mathbb{P}_{x}}\left[M_{t}\right]$ and the fact that the convergence is not a problem on bounded sets, this is verified when

$$
\int_{\mathbb{R}^{n}} e^{-t \alpha^{2}\left((1-\beta) U_{\alpha}(x)\right)^{2-\frac{2}{\alpha}}} \psi\left(e^{U_{\alpha}(x)} e^{c_{\alpha} t}\right) d x<+\infty .
$$

The $\Delta_{2}$ property of $\psi$ gives the conclusion since when $a \geq 1$ and $b$ is large $\psi(a b) \leq k^{1+\log (a) / \log (2)} \psi(b)$.

As in [21, Theorem 2.8] we show that $\mathbf{P}_{t}^{\alpha}\left(e^{U_{\alpha}}\right) \in \mathbb{L}_{\tau}\left(\nu_{\alpha}^{\otimes n}\right)$ is also a sufficient condition for $\tau$-Orlicz hyperboundedness.

Theorem 2. Let $\tau$ be as in Theorem 1. Let $t>0$. If $\mathbf{P}_{t}^{\alpha}\left(e^{U_{\alpha}}\right) \in \mathbb{L}_{\tau}\left(\nu_{\alpha}^{\otimes n}\right)$ then $\left(\mathbf{P}_{s}^{\alpha}\right)_{s \geq 0}$ is $\tau$-Orlicz hyperbounded.

Proof. Recall that thanks to (3.5), $M_{t} \leq e^{c_{\alpha} t}$. On the other hand, the Brownian semi-group $\left(\mathbf{P}_{s}\right)_{s \geq 0}$ on $\mathbb{R}^{n}$ is ultracontractive and $\left\|\mathbf{P}_{s}\right\|_{\mathbb{L}^{2}(d x) \rightarrow \mathbb{L}^{\infty}(d x)}$ $=(4 \pi s)^{-\frac{n}{4}}($ see $[27])$.

Now pick some smooth function $f$ on $\mathbb{R}^{n}$ with compact support. Since $|f| e^{-U_{\alpha}} \in \mathbb{L}^{2}(d x)$ and using the Markov property, for $s>0$ and $t>0$, it holds

$$
\begin{aligned}
\mathbb{E}^{\mathbb{P}_{x}}\left[M_{t+s}\left(e^{-U_{\alpha}}|f|\right)\left(X_{t+s}\right)\right] & =\mathbb{E}^{\mathbb{P} x}\left[M_{t} \mathbb{E}^{\mathbb{P} X_{t}}\left[M_{s}\left(e^{-U_{\alpha}}|f|\right)\left(X_{s}^{\prime}\right)\right]\right] \\
& \leq e^{c_{\alpha} s} \mathbb{E}^{\mathbb{P}_{x}}\left[M_{t} \mathbf{P}_{s}\left(|f| e^{-U_{\alpha}}\right)\left(X_{t}\right)\right] \\
& \leq e^{c_{\alpha} s}(4 \pi s)^{-\frac{n}{4}}\|f\|_{\mathbb{L}^{2}\left(\nu_{\alpha}^{\otimes n}\right)} \mathbb{E}^{\mathbb{P}_{x}}\left[M_{t}\right] .
\end{aligned}
$$

Hence $\mathbf{P}_{t+s}|f| \leq e^{c_{\alpha} s}(4 \pi s)^{-\frac{n}{4}}\|f\|_{\mathbb{L}^{2}\left(\nu_{\alpha}^{\otimes n}\right)} \mathbf{P}_{t}\left(e^{U_{\alpha}}\right)$, and consequently

$$
N_{\tau}\left(\mathbf{P}_{t+s}|f|\right) \leq e^{c_{\alpha} s}(4 \pi s)^{-\frac{n}{4}} N_{\tau}\left(\mathbf{P}_{t}\left(e^{U_{\alpha}}\right)\right)\|f\|_{\mathbb{L}^{2}\left(\nu_{\alpha}^{\otimes n}\right)} .
$$


Example 2. An appropriate choice in Theorem 1 is $\psi(y)=\exp \left((\log |y|)^{2-\frac{2}{\alpha}}\right)$ for $|y|$ large enough. According to Theorems 1 and $2,\left(\mathbf{P}_{t}^{\alpha}\right)_{t \geq 0}$ is then $\tau$-Orlicz hyperbounded for $t>\frac{1}{\alpha^{2}}$.

The previous scheme of proof extends without any change to the general framework we have introduced. Let us describe the situation.

Let $\mathbf{P}_{t}$ be a $\mu$-symmetric diffusion semi-group on a space $E$ as described in Section 1 , with generator $\mathbf{L}$. For $V$ in the domain $\mathbb{D}(\mathbf{L})$ of $\mathbf{L}$, we introduce the general Boltzmann measure $d \nu_{V}=e^{-2 V} d \mu$ and assume that $\nu_{V}$ is a probability measure. Under some assumptions it is known that one can build a $\nu_{V}$-symmetric semi-group $\left(\mathbf{P}_{t}^{V}\right)_{t \geq 0}$, via

$$
\left(\mathbf{P}_{t}^{V} h\right)(x)=e^{V(x)} \mathbb{E}^{\mathbb{P}_{x}}\left[h\left(X_{t}\right) e^{-V\left(X_{t}\right)} M_{t}\right]
$$

with

$$
M_{t}=\exp \left(\int_{0}^{t}\left(\mathbf{L} V\left(X_{s}\right)-\boldsymbol{\Gamma}(V, V)\left(X_{s}\right)\right) d s\right) .
$$

In the general case these assumptions are denoted by (H.F) in [21]. Here we have chosen the usual definition

$$
\boldsymbol{\Gamma}(V, V)=\frac{1}{2}\left(\mathbf{L} V^{2}-2 V \mathbf{L} V\right) .
$$

When $E=\mathbb{R}^{n}, L=\Delta / 2$ and $\mu=d x$ each of the following conditions (among others) is sufficient for (3.12) to hold:

(i) there exists some $\psi$ such that $\psi(x) \rightarrow+\infty$ as $|x| \rightarrow+\infty$ and $\nabla V$. $\nabla \psi-\Delta \psi$ is bounded from below,

(ii) $\int|\nabla V|^{2} d \nu_{V}<+\infty$.

See e.g. [51, p.26], [21, (5.1)] for the first one, and [24] for the second one.

We introduce the analogue of (3.5):

Assumption (OB). We shall say that $V$ satisfies Assumption OB, if

(i) $V$ is bounded from below by a possibly negative constant $d$.

(ii) There exist $c \in \mathbb{R}, u_{0}>0$ and a function $G: \mathbb{R}^{+} \rightarrow \mathbb{R}^{+}$such that $G(u) \rightarrow+\infty$ as $u \rightarrow+\infty$ and $G(u) /(u+1)$ is bounded for $u \geq u_{0}$, and such that for all $x \in E$,

$$
\boldsymbol{\Gamma}(V, V)(x)-\mathbf{L} V(x) \geq G(|V(x)|)-c .
$$

Assumption $\mathrm{OB}$ ensures that the dominating term in the analogue of (3.11) is the former for $x$ large enough. 
Theorem 3. Let $\tau$ be as in Theorem 1. If $\left(\mathbf{P}_{t}\right)_{t \geq 0}$ is ultracontractive and $V$ satisfies Assumption $O B$, then the perturbed semi-group $\left(\mathbf{P}_{t}^{V}\right)_{t \geq 0}$ is $\tau$-Orlicz hyperbounded as soon as for some $C>0$

$$
\int \psi\left(e^{V}\right) e^{-C G(|V|)} d \mu<+\infty .
$$

Remark 3. Assumption OB appeared first in Rosen's work with $G(u)=$ $u^{2\left(1-\frac{1}{\alpha}\right)}[49$, Theorem 1]. It was used to derive a variant of the logarithmic Sobolev inequalities for the $|x|^{\alpha}$ Boltzmann measures on $\mathbb{R}^{n}$. Rosen proved that his condition is in a sense optimal, see his Theorem 5. He did not relate his inequality to continuity properties of the associated semi-group. Furthermore, we think that the meaning of Assumption OB is enlightened by our probabilistic approach. Rosen's results and F-Sobolev inequalities will be discussed in Section 7 .

Remark 4. If $E=\mathbb{R}^{n}, L=\Delta / 2, \mu=d x$ and $V$ goes to infinity at infinity, then the second condition in Assumption OB implies the existence of a spectral gap, see e.g. [21, Proposition 5.3.(2)].

\section{Gross theory for Orlicz hypercontractivity}

We explore the relations between Orlicz hyperboundedness and Sobolev inequalities for the underlying measure. Since we do not a priori consider a parametrized family of Orlicz functions, contrary to the family $\left(\mathbb{L}^{p} ; p \geq 2\right)$ used in Gross theory, the extension of this theory to our framework is not immediate.

In this section we assume that $\tau$ and $\tau^{*}$ are smooth and increasing on $\mathbb{R}^{+}$, hence one to one on $\mathbb{R}^{+}$. When there is no ambiguity we denote the $\mathbb{L}^{p}(\mu)$ norm of $f$ by $\|f\|_{p}$. We assume for simplicity that $\mu$ is a probability measure. The framework is the one described in the introduction.

\subsection{An Orlicz version of Høegh-Krohn and Simon Theorem}

Høegh-Krohn and Simon [34] showed that a measure $\mu$ satisfies a possibly defective logarithmic Sobolev inequality as soon as the corresponding semigroup is continuous from $\mathbb{L}^{2}(\mu)$ into $\mathbb{L}^{p}(\mu)$ for some time and some $p>2$. A proof using semi-group techniques appears in [4, Theorem 3.6]. Another proof is given in [22, Corollary 2.8].

We follow the route in [22] in order to derive a functional inequality for an Orlicz hyperbounded semi-group. The starting point is the following particular case of Inequality (2.4) in [22]: let $\mathbb{D}$ be a nice core algebra (in the case of $\mathbb{R}^{n}$ we may choose the smooth compactly supported functions plus 
constants, see [22] for details). Let $\left(\mathbf{P}_{t}\right)_{t \geq 0}$ be a $\mu$-symmetric semi-group. Then for all $t \geq 0$, for all non-negative $f \in \mathbb{D}$ with $\int f^{2} d \mu=1$, and all positive and bounded $h$,

$$
\int f^{2} \log h d \mu \leq \frac{t}{2} \mathcal{E}(f, f)+\log \int f h \mathbf{P}_{t} f d \mu .
$$

If $\mathbf{P}_{t}$ maps $\mathbb{L}^{2}$ into some $\left(\mathbb{L}_{\tau}, N_{\tau}\right)$ with operator norm $C$, we obtain

$$
\begin{aligned}
\int f^{2} \log h d \mu & \leq \frac{t}{2} \mathcal{E}(f, f)+\log \left(\|f h\|_{\tau^{*}} N_{\tau}\left(\mathbf{P}_{t} f\right)\right) \\
& \leq \frac{t}{2} \mathcal{E}(f, f)+\log C+\log \|f h\|_{\tau^{*}}
\end{aligned}
$$

Choosing $h$ such that the last term in the above sum is bounded, yields a functional inequality reminding the logarithmic Sobolev inequality. A natural choice is

$$
h(f)=\frac{\left(\tau^{*}\right)^{-1}\left(f^{2} \tau^{*}(1)\right)}{|f|},
$$

for which $I_{\tau^{*}}(|f| h)=\int \tau^{*}(1) f^{2} d \mu=\tau^{*}(1)$. It follows that $N_{\tau^{*}}(f h)=1$ and by (2.5) that $\|f h\|_{\tau^{*}} \leq \tau(1)+\tau^{*}(1)$. We have shown

Theorem 4. Let $t_{0}>0$. Let $\left(\mathbf{P}_{t}\right)_{t \geq 0}$ be a $\mu$-symmetric semi-group. If $\mathbf{P}_{t_{0}}$ is continuous from $\mathbb{L}^{2}(\mu)$ into $\left(\mathbb{L}_{\tau}(\mu), N_{\tau}\right)$ with operator norm $C\left(t_{0}, \tau\right)$, then for all $f \in \mathbb{D}$ the following defective logarithmic Orlicz Sobolev inequality holds

$$
(D L O S I) \quad \operatorname{Ent}_{\tau}(f) \leq a \mathcal{E}(f, f)+b\|f\|_{2}^{2},
$$

with $a=\frac{t_{0}}{2}, b=\log \left(C\left(t_{0}, \tau\right)\right)+\log \left(\tau(1)+\tau^{*}(1)\right)$ and

$$
\operatorname{Ent}_{\tau}(f) \stackrel{\text { def }}{=} \int f^{2} \log \left(\frac{\left(\tau^{*}\right)^{-1}\left(\tau^{*}(1)\left(f /\|f\|_{2}\right)^{2}\right)}{\left|f /\|f\|_{2}\right|}\right) d \mu
$$

provided the function

$$
y \mapsto y^{2} \log \left(\frac{\left(\tau^{*}\right)^{-1}\left(y^{2} \tau^{*}(1)\right)}{|y|}\right)
$$

can be continuously extended up to the origin. Here $\left(\tau^{*}\right)^{-1}$ is the inverse function of $\tau^{*}$ and not $1 / \tau^{*}$.

In particular if $\left(\mathbf{P}_{t}\right)_{t \geq 0}$ is $\tau$-Orlicz hypercontractive and $\tau(1)+\tau^{*}(1)=1$, (DLOSI) is tight i.e. becomes

$$
(T L O S I) \quad \operatorname{Ent}_{\tau}(f) \leq a \mathcal{E}(f, f) .
$$

Inequalities like (DLOSI) were already discussed in the literature, as a particular $F$-Sobolev inequalities. 
Remark 5. In our setting $\left(\tau^{*}\right)^{-1}(y)>>\sqrt{y}$. Hence $\operatorname{Ent}_{\tau}(f)<+\infty$ is stronger than $L^{2}$-integrability

Example 6. If we formally replace $\tau(y)$ by $y^{p}$ for some $p>2$, then $\left(\tau^{*}\right)^{-1}(y)$ behaves like $y^{1 / q}$ for the conjugate $q$ of $p$. Hence we recover the usual logarithmic Sobolev inequality as in the result by Høegh-Krohn and Simon.

Example 7. For the $|x|^{\alpha}$ Boltzmann measures, the results of the previous section (Example 2) and the above theorem provide an Orlicz Sobolev inequality. In this case we do not have a very explicit formula for $\mathbf{E n t}_{\tau}$, but only an asymptotic behavior, i.e.

$$
\log \left(\frac{\left(\tau^{*}\right)^{-1}\left(y^{2} \tau^{*}(1)\right)}{|y|}\right) \approx(\log |y|)^{2-\frac{2}{\alpha}},
$$

as $y \rightarrow+\infty$. See Example 13 in this section for details. Here $a \approx b$ means that $c a \leq b \leq C a$ for some universal constant $c$ and $C$.

The next section provides a converse to Theorem 4 in the framework of general $F$-Sobolev inequalities.

\subsection{A Gross-Orlicz Theorem}

Our main result is Theorem 6 below. It gives the equivalence between the homogeneous $F$-Sobolev inequality and the Orlicz hypercontractivity. This extends the standard theorem of Gross [31].

Recall that the probability measure $\mu$ satisfies a log-Sobolev inequality if there exists a constant $C_{L S}$ such that for any smooth enough function $f$,

$$
\int f^{2} \log \left(\frac{f^{2}}{\mu\left(f^{2}\right)}\right) d \mu \leq C_{L S} \int|\nabla f|^{2} d \mu
$$

where $\mu\left(f^{2}\right)$ is a short hand notation for $\int f^{2} d \mu$ and $|\nabla f|^{2}$ stands for $\boldsymbol{\Gamma}(f, f)$. The following theorem is the celebrated Gross Theorem ([31], see also [3]) relating this property to the hypercontractivity of the semi-group $\left(\mathbf{P}_{t}\right)_{t \geq 0}$.

Theorem 5 ([31]). Let $\mu$ be a probability measure. The following holds:

(i) Assume that $\mu$ satisfies a log-Sobolev inequality (4.2) with constant $C_{L S}$, then, for any function $f$, any $q(0)>1$,

$$
\left\|\mathbf{P}_{t} f\right\|_{q(t)} \leq\|f\|_{q(0)}
$$

where $q(t)=1+(q(0)-1) e^{4 t / C_{L S}}$.

(ii) Assume that for any function $f$,

$$
\left\|\mathbf{P}_{t} f\right\|_{q(t)} \leq\|f\|_{2}
$$

with $q(t)=1+e^{4 t / c}$ for some $c>0$. Then the probability measure $\mu$ satisfies a log-Sobolev inequality (4.2) with constant c. 
A natural extension of the log-Sobolev inequality is the homogeneous $F$ Sobolev inequality. Let $F: \mathbb{R}^{+} \rightarrow \mathbb{R}$ be a non-decreasing function satisfying $F(1)=0$. A probability measure $\mu$ satisfies an homogeneous $F$-Sobolev inequality if there exist two constants $C_{F}$ and $\widetilde{C}_{F}$ such that for any smooth enough function $f$,

$$
\int f^{2} F\left(\frac{f^{2}}{\mu\left(f^{2}\right)}\right) d \mu \leq C_{F} \int|\nabla f|^{2} d \mu+\widetilde{C}_{F} \int f^{2} d \mu .
$$

If $\widetilde{C}_{F}=0$ (resp. $\neq 0$ ) the inequality is tight (resp. defective). We shall use this terminology only when it is necessary.

We have the following result

Theorem 6 (Gross-Orlicz). Fix $p>1$. Let $F: \mathbb{R}^{+} \rightarrow \mathbb{R}$ be a $\mathcal{C}^{2}$ nondecreasing function satisfying $F(1)=0$. Define for all $q \geq 0, \tau_{q}(x):=$ $x^{p} e^{q F\left(x^{p}\right)}$.

(i) Assume that

- there exists a non negative function $k$ on $\mathbb{R}^{+}$such that for all $q \geq 0$ : $\tau_{q}^{\prime \prime} \tau_{q} \geq \frac{k(q)}{4} \tau_{q}^{\prime 2}$ (hence $\tau_{q}$ is a Young function),

- there exists a non negative function $\ell$ on $\mathbb{R}^{+}$and a constant $m \geq 0$ such that $\tau_{q}(x) F\left(x^{p}\right) \leq \ell(q) \tau_{q}(x) F\left(\tau_{q}(x)\right)+m$, for all $q \geq 0$ and all $x \geq 0$,

- the measure $\mu$ satisfies the homogeneous F-Sobolev inequality (4.3) with constants $C_{F}$ and $\widetilde{C}_{F}$.

Then, for all non-decreasing $\mathcal{C}^{1}$ functions $q: \mathbb{R}^{+} \rightarrow \mathbb{R}^{+}$with $q(0)=0$ and satisfying $q^{\prime} \leq \frac{k(q)}{\ell(q) C_{F}}$, the following holds for all $f$,

$$
N_{\tau_{q(t)}}\left(\mathbf{P}_{t} f\right) \leq e^{\frac{1}{p}\left[m q(t)+\widetilde{C}_{F} \int_{0}^{q(t)} \ell(u) d u\right]}\|f\|_{p} .
$$

(ii) Conversely assume that there exist two non-decreasing functions, $q, r: \mathbb{R}^{+} \rightarrow \mathbb{R}^{+}$, differentiable at 0 , with $q(0)=r(0)=0$, such that for any $f$,

$$
N_{\tau_{q(t)}}\left(\mathbf{P}_{t} f\right) \leq e^{r(t)}\|f\|_{p} .
$$

Then $\mu$ satisfies the following homogeneous F-Sobolev inequality: for all $f$ smooth enough

$$
\int f^{2} F\left(\frac{f^{2}}{\mu\left(f^{2}\right)}\right) d \mu \leq \frac{4(p-1)}{p q^{\prime}(0)} \int|\nabla f|^{2} d \mu+\frac{p r^{\prime}(0)}{q^{\prime}(0)} \int f^{2} d \mu .
$$


Remark 8. Note that by our assumptions on $\tau_{q}, N_{\tau_{q(t)}}(f)$ is well defined.

Furthermore when $m=0$ the previous result states that the Orlicz hypercontractivity is equivalent to the tight homogeneous $F$-Sobolev inequality $\left(\widetilde{C}_{F}=0\right)$.

Proof. We follow the general line of the original proof by Gross [31], see also [3]. It is based on differentiation.

Without loss of generality we can assume that $f$ is non negative. Then, for a general $\mathcal{C}^{1}$ non decreasing function $q: \mathbb{R}^{+} \rightarrow \mathbb{R}^{+}$satisfying $q(0)=0$, let $N(t):=N_{\tau_{q(t)}}\left(\mathbf{P}_{t} f\right)$. For simplicity, we set $T(x, p):=\tau_{p}(x)$. Then, by definition of the gauge norm (2.4) we have

$$
\int T\left(\frac{\mathbf{P}_{t} f}{N(t)}, q(t)\right) d \mu=1 \quad \forall t \geq 0 .
$$

Thus, by differentiation, we get

$$
\begin{aligned}
\frac{N^{\prime}(t)}{N^{2}(t)} \int \mathbf{P}_{t} f \partial_{1} T\left(\frac{\mathbf{P}_{t} f}{N(t)}, q(t)\right) d \mu= & \frac{1}{N(t)} \int \mathbf{L} \mathbf{P}_{t} f \partial_{1} T\left(\frac{\mathbf{P}_{t} f}{N(t)}, q(t)\right) d \mu \\
& +q^{\prime}(t) \int \partial_{2} T\left(\frac{\mathbf{P}_{t} f}{N(t)}, q(t)\right) d \mu
\end{aligned}
$$

or equivalently, if $g:=\frac{\mathbf{P}_{t} f}{N(t)}$,

$$
\frac{N^{\prime}}{N} \int g \partial_{1} T(g, q) d \mu=\int \mathbf{L} g \partial_{1} T(g, q) d \mu+q^{\prime} \int \partial_{2} T(g, q) d \mu .
$$

Here $\partial_{1}$ and $\partial_{2}$ are short hand notations for the partial derivative with respect to the first and second variable respectively.

Let us start with the proof of the second part $(i i)$ of the Theorem. For simplicity, assume that $N(0)=\|f\|_{p}=1$. Take $t=0$ in the latter equality gives

$$
p N^{\prime}(0) \int f^{p} d \mu=p \int \mathbf{L} f \cdot f^{p-1} d \mu+q^{\prime}(0) \int f^{p} F\left(f^{p}\right) d \mu,
$$

because $g(0)=f, \partial_{1} T(f, q(0))=p f^{p-1}$ and $\partial_{2} T(f, q(0))=f^{p} F\left(f^{p}\right)$ (recall that $q(0)=0$ and $N(0)=1$ ). Using the integration by parts formula $\int \mathbf{L} f \cdot \phi(f) d \mu=-\int|\nabla f|^{2} \phi^{\prime}(f) d \mu$, we get

$$
p \int \mathbf{L} f \cdot f^{p-1} d \mu=-p(p-1) \int|\nabla f|^{2} f^{p-2} d \mu=\frac{-4(p-1)}{p} \int\left|\nabla f^{p / 2}\right|^{2} d \mu .
$$

Now, it follows from the bound (4.4) that $N^{\prime}(0) \leq r^{\prime}(0)\|f\|_{p}$. This implies

$$
p r^{\prime}(0)\|f\|_{p} \int f^{p} d \mu \geq-\frac{4(p-1)}{p} \int\left|\nabla f^{p / 2}\right|^{2} d \mu+q^{\prime}(0) \int f^{p} F\left(f^{p}\right) d \mu .
$$

Since $\|f\|_{p}=1$, this achieves the proof of $(i i)$. 
The proof of part $(i)$ is more technical. By a simple computation one can check that $x \partial_{1} T(x, q)=p T(x, q)+p q x^{2 p} F^{\prime}\left(x^{p}\right) e^{q F\left(x^{p}\right)}$. Since $F$ is non decreasing and $g \geq 0$, we get when $N^{\prime}(t) \geq 0$

$$
\frac{N^{\prime}}{N} \int g \partial_{1} T(g, q) d \mu \geq \frac{p N^{\prime}}{N} \int T(g, q) d \mu=\frac{p N^{\prime}}{N} .
$$

On the other hand, using once again the integration by parts formula $\int \mathbf{L} f$. $\phi(f) d \mu=-\int|\nabla f|^{2} \phi^{\prime}(f) d \mu$, and our assumption on $\tau_{q}$,

$$
\begin{aligned}
\int \mathbf{L} g \partial_{1} T(g, q) d \mu & =-\int|\nabla g|^{2} \partial_{11} T(g, q) d \mu \\
& \leq-k(q) \int|\nabla g|^{2} \frac{\partial_{1} T(g, p)^{2}}{4 T(g, p)} d \mu \\
& =-k(q) \int|\nabla \sqrt{T(g, q)}|^{2} d \mu .
\end{aligned}
$$

Next, $\partial_{2} T(x, q)=T(x, q) F\left(x^{p}\right) \leq \ell(q) T(x, q) F(T(x, q))+m$ by hypothesis. Thus, (4.5) becomes

$$
\frac{p N^{\prime}}{N} \leq-k(q) \int|\nabla \sqrt{T(g, q)}|^{2} d \mu+\ell(q) q^{\prime} \int T(g, q) F(T(g, q)) d \mu+m q^{\prime} .
$$

Note that the right hand side of this inequality contains the three terms appearing in the homogeneous $F$-Sobolev inequality (4.3) applied to $\sqrt{T(g, q)}$ (recall that $\int(\sqrt{T(g, q)})^{2} d \mu=1$ ). In consequence, applying the homogeneous $F$-Sobolev inequality $(4.3)$ to $\sqrt{T(g, q)}$ gives

$$
\frac{p N^{\prime}}{N} \leq q^{\prime}\left(m+\widetilde{C}_{F} \ell(q)\right)+\left[-k(q)+q^{\prime} \ell(q) C_{F}\right] \int|\nabla \sqrt{T(g, q)}|^{2} d \mu .
$$

If $q^{\prime} \leq \frac{k(q)}{C_{F} \ell(q)}$, it follows that $\frac{p N^{\prime}}{N} \leq q^{\prime}\left(m+\widetilde{C}_{F} \ell(q)\right)$. This we proved when $N^{\prime}(t) \geq 0$. It is obviously true when $N^{\prime}(t)<0$. Thus by integration

$$
N(t) \leq N(0) e^{\frac{1}{p}\left[m q(t)+\widetilde{C}_{F} \int_{0}^{q(t)} \ell(u) d u\right]} .
$$

Noting that $N(0)=\|f\|_{p}$ achieves the proof.

Remark 9. Since the homogeneous $F$-Sobolev inequality (4.3) recover the log-Sobolev inequality (4.2) (with $F=\log$ and $\widetilde{C}_{F}=0$ ), it is natural to ask whether the previous Theorem recover the classical Gross Theorem or not.

So, take $F=\log$. Then, $\tau_{q}(x)=x^{p(q+1)}$,

$$
\frac{\partial}{\partial_{q}} \tau_{q}(x)=p \tau_{q}(x) \log (x)=\frac{1}{q+1} \tau_{q}(x) F\left(\tau_{q}(x)\right),
$$


and thus, we can choose $\ell(q)=\frac{1}{q+1}$ and $m=0$. Moreover, it is easy to see that $\tau_{q}^{\prime \prime} \tau_{q}=\frac{p(q+1)-1}{p(q+1)} \tau_{q}^{\prime 2} \geq \frac{p-1}{p} \tau_{q}^{\prime 2}$, hence $k(q)=4 \frac{p-1}{p}$. Applying the Theorem, we get that if $\mu$ satisfies a log-Sobolev inequality (4.2) with constant $C_{L S}$ $\left(\widetilde{C}_{F}=0\right)$, then, for any function $f$ and any $t \geq 0$,

$$
\left\|\mathbf{P}_{t} f\right\|_{p(\tilde{q}(t)+1)} \leq\|f\|_{p}
$$

where $\tilde{q}(t)=-1+e^{4 \frac{(p-1) t}{p C_{L S}}}$. The function $p(\tilde{q}(t)+1)=p e^{4 \frac{(p-1) t}{p C_{L S}}}$ is less than $q(t)=1+(p-1) e^{4 t / C_{L S}}$ of Theorem 5 .

Let us make some additional remarks on the hypotheses of the Theorem. Remark 10. Let $m_{F}:=\left|\min _{x \in(0,1)} x F(x)\right|$ and assume that $m_{F}<\infty$. With our choice of $\tau_{q}(x)$ in the Theorem, one can choose $l \equiv 1$ and $m=m_{F}$ in order to have $\tau_{q}(x) F\left(x^{p}\right) \leq \ell(q) \tau_{q}(x) F\left(\tau_{q}(x)\right)+m$.

Moreover, if $F$ is non negative, then $m_{F}=0$. Thus, in that particular case, the previous Theorem states that the Orlicz hypercontractivity is equivalent to the tight homogeneous $F$-Sobolev inequality.

Remark 11. The condition $\tau_{q}(x) F\left(x^{p}\right) \leq \ell(q) \tau_{q}(x) F\left(\tau_{q}(x)\right)+m$ is technical. It comes from our choice of $\tau_{q}=x^{p} e^{q F\left(x^{p}\right)}$. In view of the proof of Theorem 6 the most natural choice for $\tau_{q}$ would be the solution of

$$
\left\{\begin{aligned}
\frac{\partial}{\partial q} \tau_{q}(x) & =\tau_{q}(x) F\left(\tau_{q}(x)\right) \\
\tau_{0}(x) & =x^{p}
\end{aligned}\right.
$$

Unfortunately, it is not explicit in general. This is why we preferred the expression $x^{p} e^{q F\left(x^{p}\right)}$ which has the same asymptotics when $x$ tends to infinity.

Remark 12 . The hypothesis $\tau_{q}^{\prime \prime} \tau_{q} \geq \frac{k(q)}{4}{\tau_{q}^{\prime}}^{2}$ can be read as: $\tau_{q}^{1-\frac{k(q)}{4}}$ is a convex function. Note that if $x F^{\prime}(x) \rightarrow 0$ and $x F^{\prime \prime}(x) \rightarrow 0$ when $x \rightarrow 0, \tau_{q}^{1-\frac{k(q)}{4}}$ is no more convex if $k(q)>4(p-1) / p$. Thus, we cannot hope for a better exponent than $k(q)=4(p-1) / p$ (i.e. $\left.1-\frac{k(q)}{4}=\frac{1}{p}\right)$.

Now, we give a sufficient condition, involving $F$, insuring that $\tau_{q}$ satisfies that condition.

Proposition 7. Let $F: \mathbb{R}^{+} \rightarrow \mathbb{R}$ be a $\mathcal{C}^{2}$ non decreasing function satisfying $F(1)=0$. Fix $p>1$. Define for all $q \geq 0, \tau_{q}(x)=x^{p} e^{q F\left(x^{p}\right)}$. Assume that there exists a constant $k \leq 4(p-1) / p$ such that for any $x \geq 0$,

$$
x F^{\prime \prime}(x)+\left(2+\frac{p-1}{p}-\frac{k}{2}\right) F^{\prime}(x) \geq 0,
$$

Then, for any $q \geq 0, \tau_{q}$ satisfies $\tau_{q}^{\prime \prime} \tau_{q} \geq \frac{k}{4} \tau_{q}^{\prime 2}$. 
Proof. Note that for $q=0$ the conclusion is clearly true. Suppose $q>0$. It is not difficult to check that for all $x>0$,

$$
\frac{\tau_{q}^{\prime \prime}(x) \tau_{q}(x)}{\tau_{q}^{\prime 2}(x)}=1+\frac{1}{p} \frac{-1+q(p-1) x^{p} F^{\prime}\left(x^{p}\right)+p q x^{2 p} F^{\prime \prime}\left(x^{p}\right)}{\left(1+q x^{p} F^{\prime}\left(x^{p}\right)\right)^{2}} .
$$

Thus, it is enough to prove that for any $x>0$,

$$
\begin{gathered}
-\frac{1}{p}-\frac{k}{4}+1+\left(\frac{q(p-1)}{p}-2 q\left(\frac{k}{4}-1\right)\right) x F^{\prime}(x) \\
\left.+q x^{2} F^{\prime \prime}(x)-\left(\frac{k}{4}-1\right) q^{2} x^{2} F^{\prime}(x)^{2}\right) \geq 0 .
\end{gathered}
$$

Note that $-\frac{1}{p} \geq \frac{k}{4}-1$ because $k \leq 4(p-1) / p$, hence, it is sufficient to have

$$
\left(\frac{q(p-1)}{p}-2 q\left(\frac{k}{4}-1\right)\right) x F^{\prime}(x)+q x^{2} F^{\prime \prime}(x)-\left(\frac{k}{4}-1\right) q^{2} x^{2} F^{\prime}(x)^{2} \geq 0 .
$$

Since $x>0, \frac{k}{4}-1 \leq 0$ and $F^{\prime}(x)^{2} \geq 0$, it is satisfied when

$$
\left(\frac{p-1}{p}-2\left(\frac{k}{4}-1\right)\right) F^{\prime}(x)+x F^{\prime \prime}(x) \geq 0
$$

which is our condition. This achieves the proof.

\section{3. $\tau$-Entropy and $F$-Sobolev inequalities}

In the previous two subsections we put forward two relations between $F$-Sobolev inequalities and $\tau$-hyperboundedness properties. On one hand we showed that $\tau$-hyperboundedness implies an $F$-Sobolev inequality with

$$
F\left(y^{2}\right)=\log \left(\frac{\left(\tau^{*}\right)^{-1}\left(y^{2} \tau^{*}(1)\right)}{|y|}\right) .
$$

On the other hand our analogue of Gross Theorem shows that an F-Sobolev inequality implies Orlicz hyperboundedness with say $\tau(y)=y^{2} e^{F\left(y^{2}\right)}$. The goal of this paragraph is to show that these two relations are almost the same.

First, for $\tau(y)=y^{2} \psi(y)=y^{2} e^{F\left(y^{2}\right)}$ as above, it is easily seen that

$$
\left(\tau^{*}\right)^{-1}(y)=\sqrt{2 y} \eta(y),
$$

where $\eta$ goes to $+\infty$ at infinity. Furthermore (see [46, Proposition 1 (ii) p.14]), for all $y>0$

$$
y \leq \tau^{-1}(y)\left(\tau^{*}\right)^{-1}(y) \leq 2 y
$$


We apply the latter to $y=\tau(z)$ to get $\left(\psi\left(\tau^{-1}(z)\right)\right)^{\frac{1}{2}} \leq \sqrt{2} \eta(z)$ from the lefthand inequality. Since $\tau^{-1}$ is a non decreasing function and $\tau^{-1}(z) \geq c_{\varepsilon} z^{\frac{1}{2}-\varepsilon}$ for all $\frac{1}{2}>\varepsilon>0$ for some $c_{\varepsilon}$, we certainly have $\sqrt{2} \eta(z) \geq\left(\psi\left(c_{\varepsilon} z^{\frac{1}{2}-\varepsilon}\right)\right)^{\frac{1}{2}}$. Hence provided

$$
\psi(y) \geq d_{\varepsilon}\left(\psi\left(y^{\frac{2}{1-2 \varepsilon}}\right)\right)^{k_{\varepsilon}},
$$

for some positive $k_{\varepsilon}$ and $d_{\varepsilon}$, we get that at least for large $|y|$ (using condition $\left.\nabla_{2}\right), \log (\psi(y)) \leq K_{\varepsilon} \log (\eta(y))$. Also note that (4.6) furnishes

$$
\eta(z) \leq \sqrt{2 \psi\left(\tau^{-1}(z)\right)} \leq \sqrt{2 \psi(\sqrt{z})} \leq C \sqrt{\psi(z)},
$$

for $z$ large enough. Hence under (4.7) and for large $|y|$, there exists two constant $c$ and $C$ such that $c \log (\psi) \leq \log (\eta) \leq C \log (\psi)$. In addition

$$
\log \left(\frac{\left(\tau^{*}\right)^{-1}\left(y^{2} \tau^{*}(1)\right)}{|y|}\right) \approx \log \left(\eta\left(\tau^{*}(1) y^{2}\right)\right),
$$

so that for $|y|$ large enough

$$
\log \left(\frac{\left(\tau^{*}\right)^{-1}\left(y^{2} \tau^{*}(1)\right)}{|y|}\right) \approx \log \left(\psi\left(c y^{2}\right)\right)
$$

where we recall that $a \approx b$ if there exist some universal constants $c_{1}, c_{2}$ such that $c_{1} a \leq b \leq c_{2} a$.

Finally note that for a defective $F$-Sobolev inequality we may replace $F$ by $\widetilde{F}$ that behaves like $F$ at infinity, up to the modification of both constants $C_{F}$ and $\widetilde{C}_{F}$ in (4.3). Hence provided $\psi$ satisfies (4.7) we may choose

$$
F=\log (\psi) \quad \text { or } \quad F=\log (\eta) .
$$

Example 13. Consider the $|x|^{\alpha}$ Boltzmann measure. According to Example 2, Theorem 4 and the above discussion above, there exist $A$ and $B$ such that for $\int f^{2} e^{-2|y|^{\alpha}} d y=1$,

$$
\int f^{2}(y)\left(\log _{+}(|f(y)|)\right)^{2\left(1-\frac{1}{\alpha}\right)} e^{-2|y|^{\alpha}} d y \leq A \int|\nabla f|^{2} e^{-2|y|^{\alpha}} d y+B
$$

The latter is exactly the inequality shown by Rosen in [49, Theorem 1]. His proof relies on Sobolev inequalities in $\mathbb{R}^{n}$ and results on monotone operators. Of course $F=\left(\log ^{+}\right)^{\beta}$ does not satisfy the regularity assumptions in Theorem 6, so that we cannot apply it. But smoothing this function, we may obtain similar inequalities. This will be discussed in Section 7.

Example 14. We may take $F(y)=G(\log (y))$ in the general setting of Theorem 3, provided, in addition to assumption OB, $G$ satisfies $\int e^{-q G(|V|)} d \mu<$ $+\infty$, for some $q>0$, and conditions $\Delta_{2}$ and $\nabla_{2}$. 


\subsection{From hyperboundedness to hypercontractivity}

Most of the interesting properties of hyperbounded semi-groups are in fact consequences of their possible hypercontractivity. It is well known that a defective log-Sobolev inequality and a Poincaré inequality together are equivalent to a tight log-Sobolev inequality. We shall finish this section with the proof of a similar statement for $F$-Sobolev inequalities (we refer to section 5.4 for additional results).

The first statement is straight forward

Lemma 8. Let $\mu$ be a probability measure on $\mathbb{R}^{n}$. Let $F:(0,+\infty) \rightarrow \mathbb{R}$ be $\mathcal{C}^{2}$ on a neighborhood of 1 . Assume that $F(1)=0$ and that every smooth function $f$ satisfies

$$
\int f^{2} F\left(\frac{f^{2}}{\int f^{2} d \mu}\right) \leq \int|\nabla f|^{2} d \mu .
$$

Then for every smooth function $g$

$$
\left(4 F^{\prime}(1)+2 F^{\prime \prime}(1)\right) \int\left(g-\int g d \mu\right)^{2} d \mu \leq \int|\nabla g|^{2} d \mu .
$$

In other words, setting $\Phi(x)=x F(x)$, if $\Phi^{\prime \prime}(1)>0$ one has $C_{P}(\mu) \leq$ $1 /\left(2 \Phi^{\prime \prime}(1)\right)$ where $C_{P}(\mu)$ denotes the Poincaré constant.

Proof. We apply the $F$-Sobolev inequality to $f=1+\varepsilon g$ where $g$ is bounded and $\int g d \mu=0$ and we let $\varepsilon$ to zero.

Conversely, we first prove an analogue of Rothaus inequality [50]:

Lemma 9 (Rothaus-Orlicz inequality). For any bounded function $f$, denote by $\tilde{f}$ the centered $f-\int f d \mu$. If $F$ is $\mathcal{C}^{2}$ on $(0,+\infty)$ with $F(1)=0$ and satisfies

(i) $F$ is concave non decreasing, goes to infinity at $+\infty$,

(ii) $u F^{\prime}(u)$ is bounded by $K(F)$.

Then it holds

$$
\int f^{2} F\left(\frac{f^{2}}{\mu\left(f^{2}\right)}\right) d \mu \leq \int \tilde{f}^{2} F\left(\frac{\tilde{f}^{2}}{\mu\left(\tilde{f}^{2}\right)}\right) d \mu+C_{R o t}(F)\|\tilde{f}\|_{2}^{2} .
$$

Proof. We follow the proof in [4]. Again it is enough to prove the result for functions $f$ written as $f=1+t g$ for some bounded function $g$ such that $\int g d \mu=0$ and $\int g^{2} d \mu=1$. We introduce

$$
u(t)=\frac{f^{2}}{\mu\left(f^{2}\right)}=\frac{(1+t g)^{2}}{1+t^{2}}, \log A(t)=F\left(u(t)+\varepsilon^{2}\right), \log A=F\left(g^{2}+\varepsilon^{2}\right),
$$


for some $\varepsilon>0$ and define

$$
\begin{aligned}
\varphi(t) & =\int f^{2} F\left(\frac{f^{2}}{\mu\left(f^{2}\right)}+\varepsilon^{2}\right) d \mu-\int \tilde{f}^{2} F\left(\frac{\tilde{f}^{2}}{\mu\left(\tilde{f}^{2}\right)}+\varepsilon^{2}\right) d \mu \\
& =\int(1+t g)^{2} \log A(t) d \mu-t^{2} \int g^{2} \log A d \mu .
\end{aligned}
$$

The variable $\varepsilon$ is introduced in order to avoid problems near 0 . Simple calculations yield

$$
\varphi^{\prime}(t)=\int\left(2 g(1+t g) \log A(t)-2 t g^{2} \log A+(1+t g)^{2} \frac{A^{\prime}(t)}{A(t)}\right) d \mu,
$$

and

$$
\begin{aligned}
\varphi^{\prime \prime}(t)=\int & \left(2 g^{2} \log \frac{A(t)}{A}+4 g(1+t g) \frac{A^{\prime}(t)}{A(t)}\right. \\
+ & \left.(1+t g)^{2} \frac{A^{\prime \prime}(t) A(t)-A^{\prime 2}(t)}{A^{2}(t)}\right) d \mu .
\end{aligned}
$$

It is then easy to see that $\varphi(0)=F\left(1+\varepsilon^{2}\right)$ and $\varphi^{\prime}(0)=0$. Thanks to Taylor-Lagrange formula,

$$
\varphi(t)=F\left(1+\varepsilon^{2}\right)+\frac{t^{2}}{2} \varphi^{\prime \prime}(s),
$$

for some $s$. Hence what we need is an upper bound for the second derivative, since $t^{2}=\|\tilde{f}\|_{2}^{2}$.

On one hand one has for all $t$

$$
\log \frac{A(t)}{A}=F\left(u(t)+\varepsilon^{2}\right)-F\left(g^{2}+\varepsilon^{2}\right) \leq 0
$$

if $u(t) \leq g^{2}$, and

$$
\log \frac{A(t)}{A}=F\left(u(t)+\varepsilon^{2}\right)-F\left(g^{2}+\varepsilon^{2}\right) \leq F^{\prime}\left(g^{2}+\varepsilon^{2}\right)\left(u(t)-g^{2}\right),
$$

if $u(t)>g^{2}$ since $F^{\prime}$ is non-increasing.

Note that $u(t)-g^{2}=\left(1+2 t g-g^{2}\right) /\left(1+t^{2}\right) \leq 1$. For $|g| \geq 1$ we get that $u(t)-g^{2} \leq g^{2}$. Thus, in this case, $\log (A(t) / A) \leq K(F)$. For $|g|<1$, $u(t)-g^{2} \leq 1$ yields that

$$
\begin{aligned}
\int 2 g^{2} \log \frac{A(t)}{A} d \mu & \leq \int_{\substack{|g|<1, u(t)>g^{2}}} 2 g^{2} \log \frac{A(t)}{A} d \mu+\int_{\substack{|g| \geq 1, u(t)>g^{2}}} 2 g^{2} \log \frac{A(t)}{A} d \mu \\
& \leq \int_{\substack{|g|<1, u(t)>g^{2}}} 2 g^{2} F^{\prime}\left(g^{2}+\varepsilon^{2}\right)\left(u(t)-g^{2}\right) d \mu+2 K(F) \\
& \leq 4 K(F) .
\end{aligned}
$$


On the other hand,

$$
A_{1}=\int\left(4 g(1+t g) \frac{A^{\prime}(t)}{A(t)}-(1+t g)^{2}\left(\frac{A^{\prime}(t)}{A(t)}\right)^{2}\right) d \mu \leq 4 .
$$

Indeed define

$$
Z=\left(\int(1+t g)^{2}\left(\frac{A^{\prime}(t)}{A(t)}\right)^{2} d \mu\right)^{\frac{1}{2}}
$$

and remark that, just using Cauchy-Schwarz and $\int g^{2} d \mu=1, A_{1} \leq 4 Z-Z^{2}$ which is less than 4 .

It remains to control the final term $A_{2}=\int(1+t g)^{2} \frac{A^{\prime \prime}(t)}{A(t)} d \mu$. This term may be written in terms of $F$, namely

$$
A_{2}=\int(1+t g)^{2}\left[u^{\prime 2}(t)\left(F^{\prime \prime}+{F^{\prime}}^{2}\right)\left(u(t)+\varepsilon^{2}\right)+u^{\prime \prime}(t) F^{\prime}\left(u(t)+\varepsilon^{2}\right)\right] d \mu .
$$

Since $F^{\prime \prime} \leq 0$ we only look at terms involving $F^{\prime}$. Note that

$$
u^{\prime}(t)=\frac{2(1+t g)(g-t)}{\left(1+t^{2}\right)^{2}}
$$

and

$$
(1+t g)^{2} u^{\prime \prime}(t)=2 u(t)\left[\frac{\left(3 t^{2}-1\right)+2 g t\left(t^{2}-1\right)-g^{2}\left(3 t^{2}-1\right)}{\left(1+t^{2}\right)^{2}}\right] .
$$

According to assumption $(i i)$,

$$
\begin{aligned}
(1+t g)^{2} u^{\prime 2}(t) F^{\prime 2}\left(u(t)+\varepsilon^{2}\right) & =u(t)^{2} F^{\prime 2}\left(u(t)+\varepsilon^{2}\right) \frac{4(g-t)^{2}}{\left(1+t^{2}\right)^{2}} \\
& \leq K^{2}(F) \frac{4(g-t)^{2}}{\left(1+t^{2}\right)^{2}} \leq 8 K^{2}(F)\left(1+g^{2}\right)
\end{aligned}
$$

while

$$
\begin{aligned}
(1+t g)^{2} & u^{\prime \prime}(t) F^{\prime}\left(u(t)+\varepsilon^{2}\right) \\
& \leq 2 K(F)\left[\frac{3 t^{2}+1}{\left(1+t^{2}\right)^{2}}+\frac{2|g| t\left(t^{2}+1\right)}{\left(1+t^{2}\right)^{2}}+g^{2} \frac{\left(3 t^{2}+1\right)}{\left(1+t^{2}\right)^{2}}\right] \\
& \leq 6 K(F)\left(1+|g|+g^{2}\right) \leq 12 K(F)\left(1+g^{2}\right)
\end{aligned}
$$

Integrating with respect to $\mu$ yields that $A_{2}$ is uniformly bounded from above, with a bound that does not depend on $\varepsilon$. It remains to let $\varepsilon$ go to 0 .

Remark 15 . Remark that a smoothed version of $F=\left(\log _{+}\right)^{2\left(1-\frac{1}{\alpha}\right)}$ will satisfy the hypotheses of the Lemma, for $1 \leq \alpha \leq 2$ (see section 7 ). 
Remark 16. Using the notations in the previous subsection, we have seen conditions for $F=\log (\eta)$ to be an appropriate choice. In this case using the fact that $y \mapsto \sqrt{y} \eta(y)$ is concave and non decreasing, it is easy to check that $y^{2}\left(\eta^{\prime \prime} / \eta\right)(y) \leq 3 / 4$ and $y\left(\eta^{\prime} / \eta\right)(y) \leq(1 / 2) \sqrt{y}$. Though we are not exactly in the situation of the Lemma one can however check with more efforts that a similar statement for $\mathbf{E n t}_{\tau}$ is available.

To conclude this section we may state

Theorem 10. Let $\mu$ be a probability measure on a set $E$ and $\left(\mathbf{P}_{t}\right)_{t \geq 0} a$ $\mu$-symmetric semi-group. Let $F$ be as in Lemma 9. If $\mu$ satisfies a defective F-Sobolev inequality and a Poincaré inequality, i.e.

$$
\int f^{2} F\left(\frac{f^{2}}{\mu\left(f^{2}\right)}\right) d \mu \leq C_{F} \int|\nabla f|^{2} d \mu+\widetilde{C}_{F} \int f^{2} d \mu
$$

and

$$
\int\left(f-\int f d \mu\right)^{2} d \mu \leq C_{P}(F) \int|\nabla f|^{2} d \mu
$$

then $\mu$ satisfies a tight $F$-Sobolev inequality, more precisely

$$
\int f^{2} F\left(\frac{f^{2}}{\mu\left(f^{2}\right)}\right) d \mu \leq C_{F}^{\prime} \int|\nabla f|^{2} d \mu
$$

with $C_{F}^{\prime}=C_{F}+C_{P}(F)\left(\widetilde{C}_{F}+C_{R o t}(F)\right)$.

Proof. Using the notation $\tilde{f}$ as in the previous Lemma, we have

$$
\begin{aligned}
\int f^{2} F\left(\frac{f^{2}}{\mu\left(f^{2}\right)}\right) d \mu & \leq \int \tilde{f}^{2} F\left(\frac{\tilde{f}^{2}}{\mu\left(\tilde{f}^{2}\right)}\right) d \mu+C_{R o t}(F)\|\tilde{f}\|_{2}^{2} \\
& \leq C_{F} \mathcal{E}(\tilde{f}, \tilde{f})+\left(\widetilde{C}_{F}+C_{R o t}(F)\right)\|\tilde{f}\|_{2}^{2} \\
& \leq\left(C_{F}+C_{P}(F)\left(\widetilde{C}_{F}+C_{R o t}(F)\right)\right) \mathcal{E}(f, f) .
\end{aligned}
$$

\section{Sobolev inequalities}

A measure $\mu$ on $\mathbb{R}^{n}$ satisfies a logarithmic Sobolev inequality for the usual Dirichlet form if there exists a constant $C>0$ such that for every smooth function

$$
\int f^{2} \log \left(\frac{f^{2}}{\int f^{2} d \mu}\right) d \mu \leq C \int|\nabla f|^{2} d \mu .
$$

The latter can be rewritten as

$$
\int f^{2} \log f^{2} d \mu-\left(\int f^{2} d \mu\right) \log \left(\int f^{2} d \mu\right) \leq C \int|\nabla f|^{2} d \mu
$$


and also as

$$
\lim _{p \rightarrow 2^{-}} \frac{\int f^{2} d \mu-\left(\int|f|^{p} d \mu\right)^{\frac{2}{p}}}{2-p} \leq 2 C \int|\nabla f|^{2} d \mu .
$$

Each of these forms naturally leads to considering more general inequalities. We present them before studying their properties in details. We shall say that $\mu$ satisfies a homogeneous F-Sobolev inequality when every smooth function satisfies

$$
\int f^{2} F\left(\frac{f^{2}}{\int f^{2} d \mu}\right) d \mu \leq \int|\nabla f|^{2} d \mu .
$$

i.e., in this section we only consider the tight $F$-Sobolev inequality introduced in (4.3).

The measure $\mu$ is said to verify an additive $\varphi$-Sobolev inequality when for all $f$ 's

$$
\int f^{2} \varphi\left(f^{2}\right) d \mu-\left(\int f^{2} d \mu\right) \varphi\left(\int f^{2} d \mu\right) \leq \int|\nabla f|^{2} d \mu .
$$

Finally we consider the following generalization of Beckner's inequality: for every smooth $f$

$$
\sup _{p \in(1,2)} \frac{\int f^{2} d \mu-\left(\int|f|^{p} d \mu\right)^{\frac{2}{p}}}{T(2-p)} \leq \int|\nabla f|^{2} d \mu .
$$

This property was introduced by Beckner [13] for the Gaussian measure and $T(r)=r$. It was considered by Latała and Oleszkiewicz [36] for $T(r)=C r^{a}$. A recent independent paper by Wang [56] studies the general case and gives correspondences between certain homogeneous $F$-Sobolev inequalities and generalized Beckner-type inequalities (and actual equivalences for $T(r)=$ $\left.C r^{a}\right)$.

\subsection{First remarks, tightness and tensorisation}

Using the homogeneity property, Inequality (5.1) above equivalently asserts that for every smooth function $f$ with $\int f^{2} d \mu=1$, one has $\int f^{2} F\left(f^{2}\right) d \mu \leq$ $\int|\nabla f|^{2} d \mu$. It is then obvious that when $\mu$ verifies an additive $\varphi$-Sobolev inequality as (5.2) then it satisfies a homogeneous $F$-Sobolev inequality with $F=\varphi-\varphi(1)$.

Inequality (5.1) is tight (it is an equality for constant functions) whenever $F(1)=0$. Inequalities (5.2) and (5.3) are tight by construction. Big differences appear about tensorisation. The homogeneous $F$-Sobolev inequality 
need not tensorise in general. The generalized Beckner inequality (5.3) has the tensorisation property. This is established in [36] as a consequence of the following

Lemma 11. Let $\Phi:[0, \infty) \rightarrow \mathbb{R}$ having a strictly positive second derivative and such that $1 / \Phi^{\prime \prime}$ is concave. Let $\left(\Omega_{1}, \mu_{1}\right),\left(\Omega_{2}, \mu_{2}\right)$ be probability spaces. Then for any non-negative random variable $Z$ defined on the product space $(\Omega, \mu)=\left(\Omega_{1} \times \Omega_{2}, \mu_{1} \otimes \mu_{2}\right)$ with finite expectation one has

$$
\mathbb{E}_{\mu} \Phi(Z)-\Phi\left(\mathbb{E}_{\mu} Z\right) \leq \mathbb{E}_{\mu}\left(\mathbb{E}_{\mu_{1}} \Phi(Z)-\Phi\left(\mathbb{E}_{\mu_{1}} Z\right)+\mathbb{E}_{\mu_{2}} \Phi(Z)-\Phi\left(\mathbb{E}_{\mu_{2}} Z\right)\right)
$$

When $\Phi(x)=x \varphi(x)$ satisfies the hypothesis of the lemma, one can prove that the corresponding additive $\varphi$-Sobolev inequality tensorises, even for very general Dirichlet forms. In our case, we can use the properties of the square of the gradient to prove the tensorisation property for arbitrary $\Phi$.

Lemma 12. Consider for $i=1,2$ probability spaces $\left(\mathbb{R}^{n_{i}}, \mu_{i}\right)$. Assume that for $i=1,2$ and every smooth function $f: \mathbb{R}^{n_{i}} \rightarrow \mathbb{R}$ one has

$$
\int \Phi\left(f^{2}\right) d \mu_{i}-\Phi\left(\int f^{2} d \mu_{i}\right) \leq \int|\nabla f|^{2} d \mu_{i}
$$

then the measure $\mu_{1} \otimes \mu_{2}$ enjoys exactly the same property.

Proof. Let $f: \mathbb{R}^{n_{1}+n_{2}} \rightarrow \mathbb{R}$. We start with applying Inequality (5.4) in the second variable. This gives

$$
\begin{aligned}
& \int \Phi\left(f^{2}\right) d \mu_{1} d \mu_{2}=\int\left(\int \Phi\left(f^{2}(x, y)\right) d \mu_{2}(y)\right) d \mu_{1}(x) \\
& \leq \int\left(\Phi\left(\int f^{2}(x, y) d \mu_{2}(y)\right)+\int\left|\nabla_{y} f\right|^{2}(x, y) d \mu_{2}(y)\right) d \mu_{1}(x) \\
& =\int \Phi\left(g^{2}\right) d \mu_{1}+\int\left|\nabla_{y} f\right|^{2} d \mu_{1} d \mu_{2}
\end{aligned}
$$

where we have set $g(x)=\sqrt{\int f^{2}(x, y) d \mu_{2}(y)}$. Next we apply (5.4) on the first space to $g$. Note that $\int g^{2} d \mu_{1}=\int f^{2} d \mu_{1} d \mu_{2}$ and that by the CauchySchwartz inequality

$$
|\nabla g|^{2}(x)=\frac{\left|\int f(x, y) \nabla_{x} f(x, y) d \mu_{2}(y)\right|^{2}}{\int f^{2}(x, y) d \mu_{2}(y)} \leq \int\left|\nabla_{x} f\right|^{2}(x, y) d \mu_{2}(y) .
$$

Thus we get that $\int \Phi\left(g^{2}\right) d \mu_{1} \leq \Phi\left(\int f^{2} d \mu_{1} d \mu_{2}\right)+\int\left|\nabla_{x} f\right|^{2} d \mu_{1} d \mu_{2}$. Combining this with the former inequality yields the claimed $\Phi$-Sobolev inequality on the product space. 


\subsection{The notion of capacity of a set with respect to a probability measure}

There exists a wide variety of Sobolev-type inequalities in the literature. It is natural to analyze connections between them. To do so, one tries to define for each inequality an equivalent "reduced inequality", in such a way that it is easy to decide equivalences on the reduced forms. For example it is known that Sobolev inequalities involving the $\mathbb{L}^{1}$-norm of the gradient are equivalent to isoperimetric inequalities. There exists a corresponding tool for Sobolev inequalities involving $\mathbb{L}^{2}$-norms (and even $\mathbb{L}^{p}$-norms) of gradients: capacities. We refer to the book of Maz'ya [42] for more details. The classical electrostatic capacity of a set $A \subset \mathbb{R}^{n}$ is

$$
\operatorname{Cap}(A) \stackrel{\text { def }}{=} \inf \left\{\int|\nabla f(x)|^{2} d x ; f_{\mid A}=1 \text { and } f \text { has compact support }\right\}
$$

where from now on the functions appearing in the infimum are locally Lipschitz. The usual $\mathbb{L}^{2}$-Sobolev inequalities on $\mathbb{R}^{n}$ can be reduced to an inequality relating the capacity of sets to their volume. This was extended to more general measures and inequalities (see [42]). However, if one replaces the $d x$ in the latter formula by $d \nu(x)$ where $\nu$ is a finite measure, then the above capacity is zero. The appropriate notion was introduced in [12]. We recall it after a few definitions. Let $\nu$ be an absolutely continuous measure on $\mathbb{R}^{n}$. Let $A \subset \Omega$ be Borel sets, we write

$$
\begin{aligned}
\operatorname{Cap}_{\nu}(A, \Omega) & \stackrel{\text { def }}{=} \inf \left\{\int|\nabla f|^{2} d \nu ; f_{\mid A} \geq 1 \text { and }\left.f\right|_{\Omega^{c}}=0\right\} \\
& =\inf \left\{\int|\nabla f|^{2} d \nu ; \mathbb{I}_{A} \leq f \leq \mathbb{I}_{\Omega}\right\}
\end{aligned}
$$

where the equality follows from an easy truncation. If $\mu$ is a probability measure on $\mathbb{R}^{n}$, then we set for $A$ with $\mu(A) \leq 1 / 2$

$$
\begin{aligned}
\operatorname{Cap}_{\nu}(A, \mu) & \stackrel{\text { def }}{=} \inf \left\{\int|\nabla f|^{2} d \nu ; f_{\mid A} \geq 1 \text { and } \mu(f=0) \geq \frac{1}{2}\right\} \\
& =\inf \left\{\operatorname{Cap}_{\nu}(A, \Omega) ; A \subset \Omega \text { and } \mu(\Omega) \leq \frac{1}{2}\right\} .
\end{aligned}
$$

If $\mu$ is absolutely continuous, then $\operatorname{since} \operatorname{Cap}_{\nu}(A, \Omega)$ is non-increasing in $\Omega$,

$$
\operatorname{Cap}_{\nu}(A, \mu)=\inf \left\{\operatorname{Cap}_{\nu}(A, \Omega) ; A \subset \Omega \text { and } \mu(\Omega)=\frac{1}{2}\right\}
$$

We write $\operatorname{Cap}_{\mu}(A)$ for $\operatorname{Cap}_{\mu}(A, \mu)$. 
The reduction of an $\mathbb{L}^{2}$-Sobolev inequality to an inequality between capacity and measure of sets is done via level-sets decomposition. For completeness we illustrate this on the simplest possible inequality (see [42]).

Proposition 13. Let $\mu, \nu$ be absolutely continuous measures on $\mathbb{R}^{n}$ and let $\Omega \subset \mathbb{R}^{n}$. Let $C$ denote the smallest constant so that every locally Lipschitz function vanishing on $\Omega^{c}$ verifies

$$
\int f^{2} d \mu \leq C \int|\nabla f|^{2} d \nu
$$

Then $B \leq C \leq 4 B$, where $B$ is the smallest constant so that for all $A \subset \Omega$ one has $\mu(A) \leq B \operatorname{Cap}_{\nu}(A, \Omega)$.

Remark 17. The constant 4 in the above result is best possible, and is obtained by using a result of page 109 in [42]. We shall prove the result with a worse constant. We follow a simplified proof, written in page 110 of this book (this paragraph contained a small mistake which we correct below).

Proof. The fact that $B \leq C$ is obvious from the definition of capacity. The other bounds requires level-sets decomposition. First note that replacing $f$ by $|f|$ makes the inequality tighter. So we may restrict to $f \geq 0$ vanishing outside $\Omega$. Let $\rho>1$ and consider for $k \in \mathbb{Z}, \Omega_{k}=\left\{f^{2} \geq \rho^{k}\right\}$. Then

$$
\begin{aligned}
\int f^{2} d \mu & \leq \sum_{k \in \mathbb{Z}} \rho^{k+1} \mu\left(\left\{\rho^{k} \leq f^{2}<\rho^{k+1}\right\}\right) \\
& =\sum_{k \in \mathbb{Z}} \rho^{k+1}\left(\mu\left(\Omega_{k}\right)-\mu\left(\Omega_{k+1}\right)\right)=\frac{\rho-1}{\rho} \sum_{k \in \mathbb{Z}} \rho^{k+1} \mu\left(\Omega_{k}\right) .
\end{aligned}
$$

We estimate the latter measures as follows:

$$
\mu\left(\Omega_{k}\right) \leq B \operatorname{Cap}_{\nu}\left(\Omega_{k}, \Omega\right) \leq B \int\left|\nabla g_{k}\right|^{2} d \nu
$$

where we have set $g_{k}=\min \left(1,\left(\frac{f-\rho^{(k-1) / 2}}{\rho^{k / 2}-\rho^{(k-1) / 2}}\right)_{+}\right)$. Indeed this function is 1 on $\Omega_{k}$ and vanishes outside $\Omega_{k-1}$ so outside $\Omega$. Note that

$$
\int\left|\nabla g_{k}\right|^{2} d \nu=\int_{\Omega_{k-1} \backslash \Omega_{k}} \frac{|\nabla f|^{2}}{\left(\rho^{k / 2}-\rho^{(k-1) / 2}\right)^{2}} d \nu+\int_{f=\rho^{k / 2}} \frac{|\nabla f|^{2}}{\left(\rho^{k / 2}-\rho^{(k-1) / 2}\right)^{2}} d \nu .
$$

Since $f$ is locally Lipschitz, the sets $\left\{f=\rho^{k / 2}\right\} \cap\{\nabla f \neq 0\}$ are Lebesgue negligible. So the latter integral vanishes (in the rest of the paper, similar 
arguments are sometimes needed but we omit them). Thus

$$
\begin{aligned}
\int f^{2} d \mu & \leq \frac{\rho-1}{\rho} B \sum_{k} \frac{\rho^{2}}{(\sqrt{\rho}-1)^{2}} \int_{\Omega_{k-1} \backslash \Omega_{k}}|\nabla f|^{2} d \nu \\
& \leq B \rho \frac{\sqrt{\rho}+1}{\sqrt{\rho}-1} \int|\nabla f|^{2} d \nu
\end{aligned}
$$

The best choice of $\rho$ leads to a constant $(11+5 \sqrt{5}) / 2<11.1$.

Remark 18. Let us mention another possible reduction of Sobolev type inequalities to inequalities of the form $R(\Omega) \geq \psi(\mu(\Omega))$ where $R(\Omega)$ is the infimum over functions $f$ with compact support in $\Omega$ of $\int|\nabla f|^{2} d \nu / \int f^{2} d \mu$ (Rayleigh quotient). See e.g. [5, 26] where the focus is on infinite measures. Note that by Proposition 13 this criterion amounts to inequalities of the form

$$
\mu(A) \psi(\mu(\Omega)) \leq \operatorname{Cap}_{\nu}(A, \Omega)
$$

for $A \subset \Omega$. Here the interest is in the behavior of the capacity in terms of the outer set. We shall be rather interested in estimates of the form $G(\nu(A)) \leq \operatorname{Cap}_{\nu}(A, \Omega)$, that is in the dependence on the measure of the inner sets. These two approaches are rather different, and seem to be efficient in different settings.

Remark 19. Proposition 13 appears as a $n$-dimensional version of the generalized Hardy inequality (see Muckenhoupt [44]), which asserts that the best $A$ so that every smooth $f$ on $\mathbb{R}$ with $f(0)=0$ one has

$$
\int_{0}^{+\infty} f^{2} d \mu \leq A \int_{0}^{+\infty} f^{\prime 2} d \nu
$$

verifies $B \leq A \leq 4 B$ where $B=\sup _{x>0} \mu([x,+\infty)) \int_{0}^{x} \rho_{\nu}^{-1}$, and $\rho_{\nu}$ is the density of the absolute continuous part of $\nu$. Note that $\operatorname{Cap}_{\nu}([x,+\infty),[0, \infty))=$ $\left(\int_{0}^{x} \rho_{\nu}^{-1}\right)^{-1}$, so $B$ is the smallest constant so that

$$
\mu([x,+\infty)) \leq B \operatorname{Cap}_{\nu}([x,+\infty),[0, \infty))
$$

for all $x>0$. This criterion is simpler than the one in $n$ dimensions, because one can reduce to non-decreasing functions, for which level sets are half-lines.

Remark 20. It is shown in [12] that the Poincaré constant of a measure $\mu$ verifies $C / 2 \leq C_{P} \leq K C$ where $C$ is the best constant in: $\mu(A) \leq$ $C \operatorname{Cap}_{\mu}(\mathrm{A}, \mu)$ for all $A$ with $\mu(A)<1 / 2$, and $K$ is a universal constant. Proposition 13 shows that one can take $K=4$. 


\subsection{A criterion for general Beckner-type inequalities}

The aim of this section is to give a sharp criterion for inequalities of the form (5.3). Since they appear as a collection of Sobolev inequalities, the first step consists in finding a criterion for each Sobolev inequality. This was done by the first and last-named authors in the case of measures on the line. We present here a slightly weaker but more convenient formulation of Theorem 11 in [12] and its extension to arbitrary dimension.

Theorem 14. Let $p \in(1,2), \mu, \nu$ be Borel measures on $\mathbb{R}^{n}$, with $\mu\left(\mathbb{R}^{n}\right)=1$ and $d \nu(x)=\rho_{\nu}(x) d x$. Let $C$ be the optimal constant such that for every smooth $f: \mathbb{R}^{n} \rightarrow \mathbb{R}$ one has

$$
\int f^{2} d \mu-\left(\int|f|^{p} d \mu\right)^{\frac{2}{p}} \leq C \int|\nabla f|^{2} d \nu .
$$

Then $\frac{1}{2} B(p) \leq C \leq 20 B(p)$, where $B(p)$ is the optimal constant so that every Borel set $A \subset \mathbb{R}^{n}$ with $\mu(A) \leq 1 / 2$ satisfies

$$
\mu(A)\left(1-\left(1+\frac{1}{\mu(A)}\right)^{\frac{p-2}{p}}\right) \leq B(p) \operatorname{Cap}_{\nu}(A, \mu) .
$$

If $n=1$, one has $\frac{1}{2} \max \left(B_{-}(p), B_{+}(p)\right) \leq C \leq 20 \max \left(B_{-}(p), B_{+}(p)\right)$ where

$$
\begin{gathered}
B_{+}(p)=\sup _{x>m} \mu([x,+\infty))\left(1-\left(1+\frac{1}{\mu([x, \infty))}\right)^{\frac{p-2}{p}}\right) \int_{m}^{x} \frac{1}{\rho_{\nu}}, \\
B_{-}(p)=\sup _{x<m} \mu((-\infty, x])\left(1-\left(1+\frac{1}{\mu((-\infty, x])}\right)^{\frac{p-2}{p}}\right) \int_{x}^{m} \frac{1}{\rho_{\nu}},
\end{gathered}
$$

and $m$ is a median of $\mu$.

Proof. The one dimensional result follows from [12, Theorem 11 and Remark 12] which involve $1+1 /(2 \mu([x, \infty))$. In order to derive the result presented here we have used the following easy inequality, valid for $y \geq 2$, and $p \in(1,2)$,

$$
\frac{1-(1+y)^{\frac{p-2}{p}}}{1-(1+y / 2)^{\frac{p-2}{p}}} \leq \frac{\log 3}{\log 2}
$$

Note that the left hand side is monotonous in $y$ and $p$.

We turn to the $n$-dimensional part of the theorem. We use three lemmas from [12] which we recall just after this proof. We start with the lower bound 
on the constant $C$. Assume that the Sobolev inequality (5.5) is satisfied for all functions. Let $A \subset \mathbb{R}^{n}$ with $\mu(A) \leq 1 / 2$, and let $f: \mathbb{R}^{n} \rightarrow \mathbb{R}$ be locally Lipschitz, with $f \geq \mathbb{I}_{A}$ and $\mu(f=0) \geq 1 / 2$. Denote $S=\{x ; f(x) \neq 0\}$. By Inequality (5.5) and Lemma 16, one has

$$
C \int|\nabla f|^{2} d \nu \geq \sup \left\{\int f^{2} g d \mu ; g: \mathbb{R}^{n} \rightarrow(-\infty, 1), \int(1-g)^{\frac{p}{p-2}} d \mu \leq 1\right\} .
$$

In the latter supremum, the values of $g$ on $\{f=0\}$ have no incidence on the integral, but they have an incidence on the constraint. So the supremum is achieved for $g$ 's being $-\infty$ on $\{f=0\}$. Thus

$$
\begin{aligned}
C \int|\nabla f|^{2} d \nu & \geq \sup \left\{\int_{S} f^{2} g d \mu ; g: S \rightarrow(-\infty, 1), \int_{S}(1-g)^{\frac{p}{p-2}} d \mu \leq 1\right\} \\
& \geq \sup \left\{\int_{S} f^{2} g d \mu ; g: S \rightarrow[0,1), \int_{S}(1-g)^{\frac{p}{p-2}} d \mu \leq 1\right\} \\
& \geq \sup \left\{\int \mathbb{1}_{A} g \mathbb{I}_{S} d \mu ; g: S \rightarrow[0,1), \int(1-g)^{\frac{p}{p-2}} \mathbb{I}_{S} d \mu \leq 1\right\} \\
& =\mu(A)\left(1-\left(1+\frac{1-\mu(S)}{\mu(A)}\right)^{\frac{p-2}{p}}\right),
\end{aligned}
$$

where we have used $f \geq \mathbb{1}_{A}$ and Lemma 17 for the measure $d Q=\mathbb{I}_{S} d \mu$. Since $\mu(S) \leq 1 / 2$ and this is valid for any $f$ larger than 1 on $A$ and vanishing for probability $1 / 2$ one gets

$$
\mu(A)\left(1-\left(1+\frac{1}{2 \mu(A)}\right)^{\frac{p-2}{p}}\right) \leq C \operatorname{Cap}_{\nu}(A, \mu) .
$$

One concludes with Inequality (5.6).

Next we prove the upper bound on $C$. Let $f$ be a locally Lipschitz function. Let $m$ be a median of the law of $f$ under $\mu$. Set $F=f-m$, $\Omega_{+}=\{f>m\}, \Omega_{-}=\{f<m\}, F_{+}=F \mathbb{1}_{\Omega_{+}}$and $F_{-}=F \mathbb{1}_{\Omega_{-}}$. Note that $\mu\left(\Omega_{+}\right), \mu\left(\Omega_{-}\right) \leq 1 / 2$. We define the class of functions $\mathcal{I}$ by

$$
\mathcal{I}=\left\{g: \mathbb{R}^{n} \rightarrow[0,1) ; \int(1-g)^{\frac{p}{p-2}} d \mu \leq 1+(p-1)^{\frac{p}{p-2}}\right\} .
$$

Combining Lemmas 15 and 16 and observing that $F^{2}=F_{+}^{2}+F_{-}^{2}$ gives

$$
\begin{aligned}
\int f^{2} d \mu-\left(\int|f|^{p} d \mu\right)^{\frac{2}{p}} & \leq \int F^{2} d \mu-(p-1)\left(\int|F|^{p} d \mu\right)^{\frac{2}{p}} \\
& \leq \sup _{g \in \mathcal{I}} \int\left(F_{+}^{2}+F_{-}^{2}\right) g d \mu \\
& \leq \sup _{g \in \mathcal{I}} \int F_{+}^{2} g d \mu+\sup _{g \in \mathcal{I}} \int F_{-}^{2} g d \mu .
\end{aligned}
$$


Applying Proposition 13 with the measures $g d \mu$ and $d \nu$ (it is crucial here that $g \geq 0)$ gives $\int F_{+}^{2} g d \mu \leq 4 B_{g} \int\left|\nabla F_{+}\right|^{2} d \nu$, where

$$
\begin{aligned}
B_{g} & :=\sup _{A \subset \Omega_{+}} \frac{\int \mathbb{I}_{A} g d \mu}{\operatorname{Cap}_{\nu}\left(A, \Omega_{+}\right)} \leq \sup _{\mu(A) \leq \frac{1}{2}} \frac{\int \mathbb{I}_{A} g d \mu}{\operatorname{Cap}_{\nu}(A, \mu)} \leq \sup _{\mu(A) \leq \frac{1}{2}} \frac{\sup \left\{\int \mathbb{I}_{A} g d \mu ; g \in \mathcal{I}\right\}}{\operatorname{Cap}_{\nu}(A, \mu)} \\
& =\sup _{\mu(A) \leq \frac{1}{2}} \frac{\mu(A)\left(1-\left(1+\frac{(p-1)^{\frac{p}{p-2}}}{\mu(A)}\right)^{\frac{p-2}{p}}\right)}{\operatorname{Cap}_{\nu}(A, \mu)} \leq 5 B(p) .
\end{aligned}
$$

In the preceding lines we have used Lemma 17 and the inequality

$$
1-\left(1+x(p-1)^{\frac{p}{p-2}}\right)^{\frac{p-2}{p}} \leq 5\left(1-(1+x)^{\frac{p-2}{p}}\right), x \geq 2, p \in(1,2),
$$

which follows from Remark 12 of [12]. We have shown that

$$
\sup _{g \in \mathcal{I}} \int F_{+}^{2} g d \mu \leq 20 B(p) \int\left|\nabla F_{+}\right|^{2} d \nu
$$

Adding up with a similar relation for $F_{-}$leads to

$$
\begin{aligned}
\int f^{2} d \mu-\left(\int|f|^{p} d \mu\right)^{\frac{2}{p}} & \leq 20 B(p)\left(\int\left|\nabla F_{+}\right|^{2} d \nu+\int\left|\nabla F_{-}\right|^{2} d \nu\right) \\
& =20 B(p) \int|\nabla f|^{2} d \nu
\end{aligned}
$$

We list the three lemmas from [12] that we used in the previous proof.

Lemma 15. Let $p \in(1,2)$. Let $f: X \rightarrow \mathbb{R}$ be square integrable function on a probability space $(X, Q)$. Then for all $a \in \mathbb{R}$ one has

$$
\int f^{2} d Q-\left(\int|f|^{p} d Q\right)^{\frac{2}{p}} \leq \int(f-a)^{2} d Q-(p-1)\left(\int|f-a|^{p} d Q\right)^{\frac{2}{p}} .
$$

Lemma 16. Let $\varphi$ be a non-negative integrable function on a probability space $(X, P)$. Let $A>0$ and $a \in(0,1)$, then

$$
\begin{aligned}
& \int \varphi d P-A\left(\int \varphi^{a} d P\right)^{\frac{1}{a}} \\
& \quad=\sup \left\{\int \varphi g d P ; g: X \rightarrow(-\infty, 1) \text { and } \int(1-g)^{\frac{a}{a-1}} d P \leq A^{\frac{a}{a-1}}\right\} \\
& \leq \sup \left\{\int \varphi g d P ; g: X \rightarrow[0,1) \text { and } \int(1-g)^{\frac{a}{a-1}} d P \leq 1+A^{\frac{a}{a-1}}\right\} .
\end{aligned}
$$


Lemma 17. Let $a \in(0,1)$. Let $Q$ be a finite measure on a space $X$ and let $K>Q(X)$. Let $A \subset X$ be measurable with $Q(A)>0$. Then

$$
\begin{gathered}
\sup \left\{\int_{X} \mathbb{1}_{A} g d Q ; g: X \rightarrow[0,1) \text { and } \int_{X}(1-g)^{\frac{a}{a-1}} d Q \leq K\right\} \\
=Q(A)\left(1-\left(1+\frac{K-Q(X)}{Q(A)}\right)^{\frac{a-1}{a}}\right) .
\end{gathered}
$$

Theorem 14 readily implies a sharp criterion for inequalities generalizing the ones of Beckner and Latała-Oleszkiewicz.

Theorem 18. Let $T:[0,1] \rightarrow \mathbb{R}^{+}$. Let $\mu, \nu$ be a Borel measures on $\mathbb{R}^{n}$, with $\mu\left(\mathbb{R}^{n}\right)=1$ and $d \nu(x)=\rho_{\nu}(x) d x$. Let $C$ be the optimal constant such that for every smooth $f: \mathbb{R}^{n} \rightarrow \mathbb{R}$ one has

$$
\sup _{p \in(1,2)} \frac{\int f^{2} d \mu-\left(\int|f|^{p} d \mu\right)^{\frac{2}{p}}}{T(2-p)} \leq C \int|\nabla f|^{2} d \nu .
$$

Define the function

$$
\widetilde{T}(x)=\sup _{p \in(1,2)} \frac{1-x^{\frac{p-2}{p}}}{T(2-p)} .
$$

Then $\frac{1}{2} B(T) \leq C \leq 20 B(T)$, where $B(T)$ is the smallest constant so that every Borel set $A \subset \mathbb{R}^{n}$ with $\mu(A)<1 / 2$ satisfies

$$
\mu(A) \widetilde{T}\left(1+\frac{1}{\mu(A)}\right) \leq B(T) \operatorname{Cap}_{\nu}(A, \mu) .
$$

If the dimension $n=1$, then

$$
\frac{1}{2} \max \left(B_{+}(T), B_{-}(T)\right) \leq C \leq 20 \max \left(B_{+}(T), B_{-}(T)\right),
$$

where

$$
\begin{aligned}
& B_{+}(T)=\sup _{x>m} \mu([x,+\infty)) \widetilde{T}\left(1+\frac{1}{\mu([x,+\infty))}\right) \int_{m}^{x} \frac{1}{\rho_{\nu}}, \\
& B_{-}(T)=\sup _{x>m} \mu((-\infty, x]) \widetilde{T}\left(1+\frac{1}{\mu((-\infty, x])}\right) \int_{x}^{m} \frac{1}{\rho_{\nu}},
\end{aligned}
$$

and $m$ is a median of $\mu$.

Under fairly reasonable assumptions, the following lemma gives a simple expression of $\widetilde{T}$ in terms of $T$. In particular the lemma and the theorem recover the criterion for the Latała-Oleszkiewicz on the real line and extends it to any dimension. 
Lemma 19. Let $T:[0,1] \rightarrow \mathbb{R}_{+}$be non-decreasing. Then, for any $X \geq e$,

$$
\sup _{p \in(1,2)} \frac{1-X^{\frac{p-2}{p}}}{T(2-p)} \geq \frac{1}{3 T\left(\frac{1}{\log X}\right)} \text {. }
$$

If one also assumes that $x \mapsto T(x) / x$ is non-increasing, then for $X \geq e$

$$
\sup _{p \in(1,2)} \frac{1-X^{\frac{p-2}{p}}}{T(2-p)} \leq \frac{1}{T\left(\frac{1}{\log X}\right)} \text {. }
$$

Proof. Let $b=\frac{2-p}{p}$ and note that $2-p=\frac{2 b}{b+1} \leq 2 b$. Since $T$ is nondecreasing, one has

$$
\begin{aligned}
\sup _{p \in(1,2)} \frac{1-X^{\frac{p-2}{p}}}{T(2-p)} & =\sup _{b \in(0,1)} \frac{1-e^{-b \log X}}{T\left(\frac{2 b}{b+1}\right)} \geq \sup _{b \in(0,1 / 2)} \frac{1-e^{-b \log X}}{T(2 b)} \\
& \geq \frac{1-\sqrt{e}}{T\left(\frac{1}{\log X}\right)},
\end{aligned}
$$

by choosing $b=1 /(2 \log X) \leq 1 / 2$. Finally $1-\sqrt{e} \simeq 0.393 \geq \frac{1}{3}$.

For the second assertion, let $b=\frac{2-p}{p} \in(0,1), c=b \log X$ and note that $2-p=\frac{2 b}{b+1} \geq b$. Since $T$ is non-decreasing,

$$
\begin{aligned}
\sup _{p \in(1,2)} \frac{1-X^{\frac{p-2}{p}}}{T(2-p)} & =\sup _{b \in(0,1)} \frac{1-e^{-b \log X}}{T\left(\frac{2 b}{b+1}\right)} \leq \sup _{b \in(0,1)} \frac{1-e^{-b \log X}}{T(b)} \\
& \leq \max \left[\sup _{c \in(0,1]} \frac{1-e^{-c}}{T\left(\frac{c}{\log X}\right)}, \sup _{c \in(1, \log X)} \frac{1-e^{-c}}{T\left(\frac{c}{\log X}\right)}\right] .
\end{aligned}
$$

Recall that $T(x) / x$ is non-increasing. So for $c \in(0,1], T(c / \log X) \geq$ $c T(1 / \log X)$. Hence,

$$
\sup _{c \in(0,1]} \frac{1-e^{-c}}{T\left(\frac{c}{\log X}\right)} \leq \frac{1}{T\left(\frac{1}{\log X}\right)} \sup _{c \in(0,1]} \frac{1-e^{-c}}{c}=\frac{1}{T\left(\frac{1}{\log X}\right)} .
$$

When $c \geq 1$, one has $T\left(\frac{c}{\log X}\right) \geq T\left(\frac{1}{\log X}\right)$ since $T$ is non-decreasing. Thus

$$
\sup _{c \in(1, \log X)} \frac{1-e^{-c}}{T\left(\frac{c}{\log X}\right)} \leq \frac{1}{T\left(\frac{1}{\log X}\right)} \sup _{c>1}\left(1-e^{-c}\right) \leq \frac{1}{T\left(\frac{1}{\log X}\right)} .
$$

This achieves the proof. 


\subsection{Homogeneous $\boldsymbol{F}$-Sobolev inequalities}

In the next statement, we show how to derive special homogeneous $F$-Sobolev inequalities, which ignore the behavior of functions close to their average. Such inequalities appear in the work of Wang. Let us also note that any behavior of $F$ at infinity may occur.

Theorem 20. Let $D>0$ and $\rho>1$. Let $F:[0,+\infty) \rightarrow[0,+\infty)$ be a nondecreasing function. Assume that $F(x)=0$ if $x \leq 2 \rho$. Let $\mu$ be a probability measure on $\mathbb{R}^{n}$ such that every $A \subset \mathbb{R}^{n}$ with $\mu(A) \leq 1 /(2 \rho)<1 / 2$

$$
\mu(A) F\left(\frac{\rho}{\mu(A)}\right) \leq D \operatorname{Cap}_{\mu}(A)
$$

Then for every smooth $f: \mathbb{R}^{n} \rightarrow \mathbb{R}$ one has

$$
\int f^{2} F\left(\frac{f^{2}}{\int f^{2} d \mu}\right) d \mu \leq D\left(\frac{\rho}{\sqrt{\rho}-1}\right)^{2} \int|\nabla f|^{2} d \mu .
$$

Proof. For $k \geq 1$, set $\Omega_{k}=\left\{x ; f^{2}(x) \geq 2 \rho^{k} \mu\left(f^{2}\right)\right\}$. Chebyshev inequality gives $\mu\left(\Omega_{k}\right) \leq 1 /\left(2 \rho^{k}\right)$. Next, since $F$ vanishes on $[0,2 \rho]$

$$
\begin{aligned}
\int f^{2} F\left(\frac{f^{2}}{\int f^{2} d \mu}\right) d \mu & \leq \sum_{k \geq 1} \int_{\Omega_{k} \backslash \Omega_{k+1}} f^{2} F\left(\frac{f^{2}}{\int f^{2} d \mu}\right) d \mu \\
& \leq \sum_{k \geq 1} \mu\left(\Omega_{k}\right) 2 \rho^{k+1} \mu\left(f^{2}\right) F\left(2 \rho^{k+1}\right)
\end{aligned}
$$

Since $k \geq 1$ and $F$ is non-decreasing, we have

$$
\mu\left(\Omega_{k}\right) F\left(2 \rho^{k+1}\right) \leq \mu\left(\Omega_{k}\right) F\left(\frac{\rho}{\mu\left(\Omega_{k}\right)}\right) \leq D \operatorname{Cap}_{\mu}\left(\Omega_{k}\right)
$$

Let us consider the function

$$
h_{k}=\min \left(1,\left(\frac{|f|-\sqrt{2 \rho^{k-1} \mu\left(f^{2}\right)}}{\sqrt{2 \rho^{k} \mu\left(f^{2}\right)}-\sqrt{2 \rho^{k-1} \mu\left(f^{2}\right)}}\right)_{+}\right)
$$

it is equal to 1 on $\Omega_{k}$ and zero outside $\Omega_{k-1}$. Since for $k \geq 1, \mu\left(\Omega_{k-1}\right) \leq 1 / 2$, $h_{k}$ vanishes with probability at least $1 / 2$. Thus

$$
\operatorname{Cap}_{\mu}\left(\Omega_{k}\right) \leq \int\left|\nabla h_{k}\right|^{2} d \mu=\frac{\int_{\Omega_{k-1} \backslash \Omega_{k}}|\nabla f|^{2} d \mu}{2 \rho^{k-1}(\sqrt{\rho}-1)^{2} \mu\left(f^{2}\right)}
$$


Combining these estimates gives

$$
\begin{aligned}
\int f^{2} F\left(\frac{f^{2}}{\int f^{2} d \mu}\right) d \mu & \leq D \sum_{k \geq 1} 2 \rho^{k+1} \mu\left(f^{2}\right) \operatorname{Cap}_{\mu}\left(\Omega_{k}\right) \\
& \leq D\left(\frac{\rho}{\sqrt{\rho}-1}\right)^{2} \int|\nabla f|^{2} d \mu .
\end{aligned}
$$

In the following we briefly study homogeneous F-Sobolev inequalities which are tight but do not ignore the values of functions close to their $\mathbb{L}^{2}$ norm. In this case the behavior of $F$ at 1 is crucial. We have already seen in Lemma 8 of Section 4.4 that when $F(1)=0$ and $x \mapsto x F(x)$ has a positive second derivative at 1 , then the homogeneous $F$-Sobolev inequality implies a spectral inequality. Besides, if a measure satisfies a Poincaré inequality, and a tight homogeneous $F$-Sobolev inequality which ignores small values of functions, then one can modify $F$ on small values in an almost arbitrary way:

Lemma 21. Let $D>0$ and $\rho>1$. Let $F:[0,+\infty) \rightarrow \mathbb{R}$ be a non-decreasing function, such that $F=0$ on $[0,2 \rho)$. Let $\mu$ be a probability measure on $\mathbb{R}^{n}$ with Poincaré constant $C_{P}<\infty$ and such that every smooth function $f$ on $\mathbb{R}^{n}$ satisfies

$$
\int f^{2} F\left(\frac{f^{2}}{\int f^{2} d \mu}\right) d \mu \leq D \int|\nabla f|^{2} d \mu
$$

Let $\widetilde{F}:[0,+\infty) \rightarrow \mathbb{R}$ be non-decreasing such that $\widetilde{F}(1)=0, \widetilde{F}$ is $C^{2}$ on $[0,2 \rho]$ and $\widetilde{F}(x)=F(x)$ for $x \geq 2 \rho$. Set $\Phi(x)=x \widetilde{F}(x)$. Then for every smooth $f: \mathbb{R}^{n} \rightarrow \mathbb{R}$ one has

$$
\int f^{2} \widetilde{F}\left(\frac{f^{2}}{\int f^{2} d \mu}\right) d \mu \leq\left((1+\sqrt{2 \rho})^{2} C_{P}\left(\max _{[0,2 \rho]} \Phi^{\prime \prime}\right)_{+}+D\right) \int|\nabla f|^{2} d \mu .
$$

Proof. Note that $\Phi(1)=0$ and $\Phi^{\prime}(1)=\widetilde{F}^{\prime}(1) \geq 0$. We introduce the function $\Phi_{1}(x)=\Phi(x)-\Phi(1)-\Phi^{\prime}(1)(x-1)$. Without loss of generality, we consider a function $f \geq 0$ with $\int f^{2} d \mu=1$. One has

$$
\int \Phi\left(f^{2}\right) d \mu=\int \Phi_{1}\left(f^{2}\right) d \mu=\int_{f^{2} \leq 2 \rho} \Phi_{1}\left(f^{2}\right) d \mu+\int_{f^{2}>2 \rho} \Phi_{1}\left(f^{2}\right) d \mu .
$$

For the first term, using Taylor's formula and $0 \leq f \leq \sqrt{2 \rho}$, we obtain

$$
\Phi_{1}\left(f^{2}\right) \leq\left(\max _{[0,2 \rho]} \Phi^{\prime \prime}\right) \frac{\left(f^{2}-1\right)^{2}}{2} \leq \frac{(1+\sqrt{2 \rho})^{2}}{2}\left(\max _{[0,2 \rho]} \Phi^{\prime \prime}\right)_{+}(f-1)^{2} .
$$


Therefore

$$
\int_{f^{2} \leq 2 \rho} \Phi_{1}\left(f^{2}\right) d \mu \leq \frac{(1+\sqrt{2 \rho})^{2}}{2}\left(\max _{[0,2 \rho]} \Phi^{\prime \prime}\right)_{+} \int(f-1)^{2} d \mu
$$

can be upper-bounded thanks to the Poincaré inequality. Indeed

$$
\begin{aligned}
\int(f-1)^{2} d \mu & =\int\left(f-\mu\left(f^{2}\right)^{\frac{1}{2}}\right)^{2} \\
& =2\left(\int f^{2} d \mu-\int f d \mu\left(\int f^{2} d \mu\right)^{\frac{1}{2}}\right) \\
& \leq 2\left(\int f^{2} d \mu-\left(\int f d \mu\right)^{2}\right) \leq 2 C_{P} \int|\nabla f|^{2} d \mu .
\end{aligned}
$$

The second term in (5.8) is easily handled by our hypothesis. Indeed, since $\Phi^{\prime}(1) \geq 0$

$$
\int_{f^{2}>2 \rho} \Phi_{1}\left(f^{2}\right) d \mu \leq \int_{f^{2}>2 \rho} \Phi\left(f^{2}\right) d \mu \leq \int f^{2} F\left(f^{2}\right) d \mu \leq D \int|\nabla f|^{2} d \mu .
$$

Finally, we show that an homogeneous $F$-Sobolev inequality implies an inequality between capacity and measure. We believe that the result should be true in more generality.

Theorem 22. Let $\mu$ be a probability measure on $\mathbb{R}^{n}$. Let $F: \mathbb{R}^{+} \rightarrow \mathbb{R}^{+}$be a non-negative non-decreasing function such that there exists $\lambda \geq 4$ such that for $x \geq 2, F(x) / x$ is non-increasing and $F(\lambda x) \leq \lambda F(x) / 4$. Assume that for every smooth function, one has

$$
\int f^{2} F\left(\frac{f^{2}}{\mu\left(f^{2}\right)}\right) d \mu \leq D \int|\nabla f|^{2} d \mu
$$

then for all $A \subset \mathbb{R}^{n}$ with $\mu(A) \leq \frac{1}{2}$ it holds

$$
\mu(A) F\left(\frac{1}{\mu(A)}\right) \leq 4 \lambda D \operatorname{Cap}_{\mu}(A) .
$$

Proof. Let $A$ be a set of measure less than $1 / 2$. In order to estimate its capacity, we may consider non-negative functions $g \geq \mathbb{I}_{A}$ and $\mu(g=0) \geq$ $1 / 2$. For $k \in \mathbb{Z}$ we consider the function

$$
g_{k}=\min \left(\left(g-2^{k} \sqrt{\mu\left(g^{2}\right)}\right)_{+}, 2^{k} \sqrt{\mu\left(g^{2}\right)}\right) .
$$


We also set $\Omega_{k}=\left\{x ; g(x) \geq 2^{k} \sqrt{\mu\left(g^{2}\right)}\right\}$. Note that on $\Omega_{k+1}, g_{k}^{2}$ is constantly $2^{2 k} \mu\left(g^{2}\right)$ and that $\int g_{k}^{2} d \mu \leq \mu\left(\Omega_{k}\right) 2^{2 k} \mu\left(g^{2}\right)$. Therefore, applying the $F$-Sobolev inequality (with $F \geq 0$ ) to $g_{k}$ yields

$$
\begin{aligned}
D \int|\nabla g|^{2} d \mu & \geq D \int\left|\nabla g_{k}\right|^{2} d \mu \geq \int_{\Omega_{k+1}} g_{k}^{2} F\left(\frac{g_{k}^{2}}{\mu\left(g_{k}^{2}\right)}\right) d \mu \\
& \geq \mu\left(\Omega_{k+1}\right) 2^{2 k} \mu\left(g^{2}\right) F\left(\frac{1}{\mu\left(\Omega_{k}\right)}\right) .
\end{aligned}
$$

Setting $a_{k}=\mu\left(\Omega_{k}\right)$ and $C=D \int|\nabla g|^{2} d \mu / \mu\left(g^{2}\right)$, we have for $k \in \mathbb{Z}$

$$
2^{2 k} a_{k+1} F\left(1 / a_{k}\right) \leq C
$$

Lemma 23 guarantees that $2^{2 k} a_{k} F\left(1 / a_{k}\right) \leq \lambda C$ for every $k$ with $a_{k}>0$, that is

$$
2^{2 k} \mu\left(g^{2}\right) \mu\left(\Omega_{k}\right) F\left(\frac{1}{\mu\left(\Omega_{k}\right)}\right) \leq \lambda D \int|\nabla g|^{2} d \mu .
$$

We choose the largest $k$ with $2^{k} \sqrt{\mu\left(g^{2}\right)} \leq 1$. Thus $2^{k+1} \sqrt{\mu\left(g^{2}\right)}>1$ and $A \subset \Omega_{k}$. In particular $2 \leq 1 / \mu\left(\Omega_{k}\right) \leq 1 / \mu(A)$, so these ratios are in the range where $x \mapsto F(x) / x$ is non-increasing. Combining these remarks with the above inequality yields

$$
\frac{1}{4} \mu(A) F\left(\frac{1}{\mu(A)}\right) \leq \lambda D \int|\nabla g|^{2} d \mu
$$

Since this is valid for every $g \geq \mathbb{1}_{A}$ and vanishing on a set of measure at least $1 / 2$, we have shown that $\mu(A) F(1 / \mu(A)) \leq 4 \lambda D \operatorname{Cap}_{\mu}(A)$.

The next lemma was inspired by the argument of Theorem 10.5 in [5].

Lemma 23. Let $F:[2,+\infty) \rightarrow[0,+\infty)$ be a non-decreasing function such that $x \rightarrow F(x) / x$ is non increasing and there exists $\lambda \geq 4$ such that for all $x \geq 2$ one has $F(\lambda x) \leq \lambda F(x) / 4$. Let $\left(a_{k}\right)_{k \in \mathbb{Z}}$ be a non-increasing (doublesided) sequence of numbers in $[0,1 / 2]$. Assume that for all $k \in \mathbb{Z}$ with $a_{k}>0$ one has

$$
2^{2 k} a_{k+1} F\left(\frac{1}{a_{k}}\right) \leq C,
$$

then for all $k \in \mathbb{Z}$ with $a_{k}>0$ one has

$$
2^{2 k} a_{k} F\left(\frac{1}{a_{k}}\right) \leq \lambda C
$$


Proof. Discarding trivial cases where $F\left(1 / a_{k}\right)$ is always zero, we observe that the sequence $2^{2 k} F\left(1 / a_{k}\right)$ tends to $+\infty$ when $k$ tends to $+\infty$, and tends to zero when $k$ tends to $-\infty$. So we define $k_{0}$ as the largest integer such that $2^{2 k} F\left(1 / a_{k}\right) \leq 2 C$. Let $k \leq k_{0}$, then $2 C \geq 2{ }^{2 k} F\left(1 / a_{k}\right) \geq 2{ }^{2 k} F(2)$ since $a_{k} \leq 1 / 2$ and $F$ is non-decreasing. Moreover since $F(t) / t$ is non-increasing, we also have

$$
2^{2(k+1)} a_{k+1} F\left(\frac{1}{a_{k+1}}\right) \leq 2^{2(k+1)} F(2) / 2 .
$$

Combining these two inequalities yields

$$
2^{2(k+1)} a_{k+1} F\left(\frac{1}{a_{k+1}}\right) \leq 4 C \leq \lambda C,
$$

so the claimed result is established for $k \leq k_{0}+1$. For larger values we proceed by induction. Let $k \geq k_{0}+1$, for which the conclusion holds. If $a_{k+1}=0$ we have nothing to prove. Otherwise the hypothesis of the lemma gives

$$
\frac{1}{a_{k+1}} \geq \frac{2^{2 k} F\left(\frac{1}{a_{k}}\right)}{C} .
$$

Since $k>k_{0}$ we know that the term on the right is larger than 2. Using the fact that $t \geq 2 \mapsto F(t) / t$ is non-increasing, we obtain

$$
a_{k+1} F\left(\frac{1}{a_{k+1}}\right) \leq \frac{C}{2^{2 k} F\left(\frac{1}{a_{k}}\right)} F\left(\frac{2^{2 k} F\left(\frac{1}{a_{k}}\right)}{C}\right) .
$$

Next, by the induction hypothesis for $k$ this is bounded from above by

$$
\frac{C}{2^{2 k} F\left(\frac{1}{a_{k}}\right)} F\left(\frac{\lambda}{a_{k}}\right) \leq \frac{C}{2^{2 k}} \cdot \frac{\lambda}{4}
$$

where we have used $F(\lambda t) \leq \lambda F(t) / 4$. So we have shown

$$
a_{k+1} F\left(\frac{1}{a_{k+1}}\right) \leq 2^{-2 k-2} \lambda C,
$$

and the conclusion is valid for $k+1$.

Remark 21. The alternative reduction of Sobolev type inequalities to estimates on the Rayleigh quotient (see Remark 18) turns out to work better for homogeneous F-Sobolev inequalities. See Proposition 2.2 in [26], dealing with measures of infinite mass, but the proof of which extends to our setting. 
Remark 22. Applying Theorem 22 to the function $F=\mathbb{1}_{[2,+\infty)}$ and $\lambda=4$ shows the following. If for every function one has

$$
\int_{f^{2} \geq 2 \mu\left(f^{2}\right)} f^{2} d \mu \leq C \int|\nabla f|^{2} d \mu
$$

then for all $A \subset \mathbb{R}^{n}$ with $\mu(A) \leq 1 / 2$, one has $\mu(A) \leq \operatorname{16CCap}_{\mu}(A)$. By Remark 20, the measure $\mu$ satisfies a Poincaré inequality with constant $C_{P}(\mu) \leq 64 C$.

The converse implication also holds. Assume that $\mu$ satisfies, for all $f$, $\operatorname{Var}_{\mu}(f) \leq C_{P}(\mu) \int|\nabla f|^{2} d \mu$. Without loss of generality, we consider $f \geq 0$. If $f^{2} \geq 2 \mu\left(f^{2}\right)$ then by Cauchy-Schwarz one has $f \geq \sqrt{2} \mu(f)$ and consequently $(f-\mu(f))^{2} \geq(1-1 / \sqrt{2})^{2} f^{2}$.

Hence $\operatorname{Var}_{\mu}(f) \geq(1-1 / \sqrt{2})^{2} \int_{f^{2} \geq 2 \mu\left(f^{2}\right)} f^{2} d \mu$ and the Poincaré inequality implies

$$
\int_{f^{2} \geq 2 \mu\left(f^{2}\right)} f^{2} d \mu \leq\left(1-\frac{1}{\sqrt{2}}\right)^{-2} C_{P}(\mu) \int|\nabla f|^{2} d \mu \leq 12 C_{P}(\mu) \int|\nabla f|^{2} d \mu .
$$

As a conclusion, Poincaré inequality enters the framework of homogeneous $F$-Sobolev inequalities and is equivalent to

$$
\int f^{2} 1_{f^{2} \geq 2 \mu\left(f^{2}\right)} d \mu \leq C \int|\nabla f|^{2} d \mu
$$

(up to the constants). Note that the number 2 is crucial in our argument.

Remark 23. Let us present a convenient variant of Theorem 22. Assume that $\mu$ satisfies a Poincaré inequality and a $F$-Sobolev inequality as in Theorem 22. If $F$ verifies the assumptions $F(x) / x$ non-increasing and $F(\lambda x) \leq$ $\lambda F(x) / 4$ only for $x \geq x_{0}>2$ then one can however conclude with a similar inequality between capacity and measure. To see this, introduce a function $\widetilde{F}$ on $\mathbb{R}^{+}$with $\widetilde{F}(x):=F(x)$ for $x \geq x_{0}, \widetilde{F}(x):=F\left(x_{0}\right)$ for $x \in\left[2, x_{0}\right], \widetilde{F}(1)=0$ and $\widetilde{F}$ is $C^{2}$ and non-decreasing on $\left[0, x_{0}\right]$. Then by Lemma $21, \mu$ satisfies a homogeneous $\widetilde{F}$-Sobolev inequality, and $\widetilde{F}$ satisfies the assumptions of Theorem 22. Therefore one obtains an inequality of the form $\mu(A) \widetilde{F}(1 / \mu(A)) \leq K \operatorname{Cap}_{\mu}(A)$. In particular if $\mu(A) \leq 1 / x_{0}$ one has $\mu(A) F(1 / \mu(A)) \leq K \operatorname{Cap}_{\mu}(A)$.

\subsection{Additive $\phi$-Sobolev inequalities}

We present an extension of a method developed by Miclo and Roberto [43] for logarithmic Sobolev inequalities. Throughout this section, we work with a function $\Phi(x)=x \varphi(x)$, where $\varphi:(0,+\infty) \rightarrow \mathbb{R}$ is non-decreasing, continuously differentiable. We assume that $\Phi$ can be extended to 0 . For $x, t>0$ 
we define the function

$$
\Phi_{t}(x)=\Phi(x)-\Phi(t)-\Phi^{\prime}(t)(x-t)=x(\varphi(x)-\varphi(t))-t \varphi^{\prime}(t)(x-t) .
$$

We start with two preliminary statements about $\Phi$-entropies. The first one is classical and easy, and we skip its proof (see also Lemma 3.4.2 in [3]). For short, we write $\mu(g)$ for $\int g d \mu$.

Lemma 24. For every function $f$,

$$
\int \Phi\left(f^{2}\right) d \mu-\Phi\left(\int f^{2} d \mu\right)=\int \Phi_{\mu\left(f^{2}\right)}\left(f^{2}\right) d \mu .
$$

When $\Phi$ is convex, one has

$$
\int \Phi\left(f^{2}\right) d \mu-\Phi\left(\int f^{2} d \mu\right)=\inf _{t>0} \int \Phi_{t}\left(f^{2}\right) d \mu .
$$

Lemma 25. Let the function $\varphi$ be non-decreasing and concave. Assume that there exists $\gamma \geq 0$ such that $y \varphi^{\prime}(y) \leq \gamma$ for all $y>0$. Then for every $t>0$ and every $x \in[0,2 t]$ one has

$$
\Phi_{t^{2}}\left(x^{2}\right) \leq 9 \gamma(x-t)^{2} .
$$

Proof. The concavity of $\varphi$ ensures that $\varphi\left(x^{2}\right) \leq \varphi\left(t^{2}\right)+\varphi^{\prime}\left(t^{2}\right)\left(x^{2}-t^{2}\right)$. This yields

$$
\begin{aligned}
\Phi_{t^{2}}\left(x^{2}\right) & \leq \varphi^{\prime}\left(t^{2}\right)\left(x^{2}-t^{2}\right)^{2}=(x-t)^{2} \varphi^{\prime}\left(t^{2}\right)(x+t)^{2} \\
& \leq(x-t)^{2} \varphi^{\prime}\left(t^{2}\right)(3 t)^{2} \leq 9 \gamma(x-t)^{2},
\end{aligned}
$$

where we have used $x \leq 2 t$.

Theorem 26. Let $\varphi$ be a non-decreasing, concave, $C^{1}$ function on $(0,+\infty)$ with $\varphi(8)>0$. Assume that there exist constants $\gamma, M$ such that for all $x, y>0$ one has

$$
x \varphi^{\prime}(x) \leq \gamma \quad \text { and } \quad \varphi(x y) \leq M+\varphi(x)+\varphi(y) .
$$

Let $\mu$ be a probability measure on $\mathbb{R}^{n}$ satisfying a Poincaré inequality with constant $C_{P}$ and the following relation between capacity and measure: there exists $D>0$ such that for all $A \subset \mathbb{R}^{n}$ with $\mu(A)<1 / 4$

$$
\mu(A) \varphi\left(\frac{2}{\mu(A)}\right) \leq \operatorname{DCap}_{\mu}(A)
$$

then for every smooth function one has

$$
\int \Phi\left(f^{2}\right) d \mu-\Phi\left(\int f^{2} d \mu\right) \leq\left(18 \gamma C_{P}+24\left(1+\frac{M}{\varphi(8)}\right) D\right) \int|\nabla f|^{2} d \mu
$$

where as usual $\Phi(x)=x \varphi(x)$. 
Proof. Without loss of generality, we may consider $f \geq 0$. Set $t=\left(\mu\left(f^{2}\right)\right)^{\frac{1}{2}}$. Then

$$
\begin{aligned}
\int \Phi\left(f^{2}\right) d \mu-\Phi\left(\int f^{2} d \mu\right) & =\int \Phi_{t^{2}}\left(f^{2}\right) d \mu \\
& =\int_{f^{2} \leq 4 \mu\left(f^{2}\right)} \Phi_{t^{2}}\left(f^{2}\right) d \mu+\int_{f^{2}>4 \mu\left(f^{2}\right)} \Phi_{t^{2}}\left(f^{2}\right) d \mu .
\end{aligned}
$$

The first term is bounded from above thanks to Lemma 25, indeed

$$
\begin{aligned}
\int_{f^{2} \leq 4 \mu\left(f^{2}\right)} & \Phi_{t^{2}}\left(f^{2}\right) d \mu=\int_{f \in[0,2 t]} \Phi_{t^{2}}\left(f^{2}\right) \\
& \leq 9 \gamma \int_{f \in[0,2 t]}\left(f-\mu\left(f^{2}\right)^{\frac{1}{2}}\right)^{2} d \mu \leq 9 \gamma \int\left(f-\mu\left(f^{2}\right)^{\frac{1}{2}}\right)^{2} \\
& =18 \gamma\left(\int f^{2} d \mu-\int f d \mu\left(\int f^{2} d \mu\right)^{\frac{1}{2}}\right) \\
& \leq 18 \gamma\left(\int f^{2} d \mu-\left(\int f d \mu\right)^{2}\right) \leq 18 \gamma C_{P} \int|\nabla f|^{2} d \mu,
\end{aligned}
$$

where we have used Cauchy-Schwartz and the Poincaré inequality for $\mu$.

The second term in (5.9) is estimated as follows

$$
\begin{aligned}
\int_{f^{2}>4 \mu\left(f^{2}\right)} \Phi_{t^{2}}\left(f^{2}\right) d \mu & =\int_{f^{2}>4 \mu\left(f^{2}\right)}\left[f^{2}\left(\varphi\left(f^{2}\right)-\varphi\left(\mu\left(f^{2}\right)\right)\right)\right. \\
& \left.-\mu\left(f^{2}\right) \varphi^{\prime}\left(\mu\left(f^{2}\right)\right)\left(f^{2}-\mu\left(f^{2}\right)\right)\right] d \mu \\
& \leq \int_{f^{2}>4 \mu\left(f^{2}\right)} f^{2}\left(\varphi\left(f^{2}\right)-\varphi\left(\mu\left(f^{2}\right)\right)\right) d \mu \\
& \leq \int_{f^{2}>4 \mu\left(f^{2}\right)} f^{2}\left(\varphi\left(\frac{f^{2}}{\mu\left(f^{2}\right)}\right)+M\right) d \mu
\end{aligned}
$$

We conclude by applying Theorem 20 with $\rho=2, F(x)=0$ if $x \leq 4$, and $F(x)=\varphi(x)+M$ if $x>4$. Since for $\mu(A) \leq 1 / 4$ one has

$$
\begin{aligned}
\mu(A) F\left(\frac{2}{\mu(A)}\right) & =\mu(A) \varphi\left(\frac{2}{\mu(A)}\right)\left(1+\frac{M}{\varphi\left(\frac{2}{\mu(A)}\right)}\right) \\
& \leq D\left(1+\frac{M}{\varphi(8)}\right) \operatorname{Cap}_{\mu}(A),
\end{aligned}
$$

we obtain

$$
\int_{f^{2}>4 \mu\left(f^{2}\right)} \Phi_{t^{2}}\left(f^{2}\right) d \mu \leq 4(\sqrt{2}+1)^{2} D\left(1+\frac{M}{\varphi(8)}\right) \int|\nabla f|^{2} d \mu .
$$


Remark 24. As already explained, the Poincaré constant of the measure $\mu$ is bounded above by $4 B$ where $B$ is the best constant such that every set $A$ with $\mu(A) \leq 1 / 2$ verifies $\mu(A) \leq B \operatorname{Cap}_{\mu}(A)$. If $\varphi(4)>0$, one has

$$
D:=\sup _{\mu(A) \leq 1 / 2} \frac{\mu(A) \varphi(2 / \mu(A))}{\operatorname{Cap}_{\mu}(A)} \geq \varphi(4) \sup _{\mu(A) \leq 1 / 2} \frac{\mu(A)}{\operatorname{Cap}_{\mu}(A)}=\varphi(4) B .
$$

So $C_{P} \leq 4 D / \varphi(4)$. In particular, if $D<+\infty$, then $\mu$ satisfies an additive $\phi$-Sobolev inequality.

Remark 25. As already mentioned, the additive $\varphi$-Sobolev inequality has the tensorisation property. If it is valid for a measure $\mu$ (with second moment) then it is true for its product measures, and by a classical application of the Central Limit Theorem it holds for the Gaussian measure. For the latter it is known that the logarithmic Sobolev inequality, viewed as an embedding result, is optimal. So $\varphi$ cannot grow faster than a logarithm. Note that both hypothesis on $\varphi$ assumed in Theorem 26 imply that $\varphi$ is at most a logarithm.

Next we present an improved criterion for measures on the real line.

Theorem 27. Let $\Phi$ be a continuous convex function on $[0, \infty)$, with $\Phi(x)=$ $x \varphi(x)$ for $x>0$. Assume that $\varphi$ is non-decreasing, concave, and $C^{1}$ on $(0,+\infty)$ with $\varphi(8)>0$. Assume that there exist constants $\gamma, M$ such that for all $x, y>0$ one has

$$
x \varphi^{\prime}(x) \leq \gamma \quad \text { and } \quad \varphi(x y) \leq M+\varphi(x)+\varphi(y) .
$$

Let $\mu$ be a probability measure on $\mathbb{R}$, with density $\rho_{\mu}$, and median $m$. Let

$$
\begin{aligned}
& D_{+}=\sup _{x>m} \mu([x,+\infty)) \varphi\left(\frac{2}{\mu([x,+\infty))}\right) \int_{m}^{x} \frac{1}{\rho_{\mu}} \\
& D_{-}=\sup _{x<m} \mu((-\infty, x]) \varphi\left(\frac{2}{\mu((-\infty, x])}\right) \int_{x}^{m} \frac{1}{\rho_{\mu}} \\
& B_{+}=\sup _{x>m} \mu([x,+\infty)) \int_{m}^{x} \frac{1}{\rho_{\mu}} \\
& B_{-}=\sup _{x<m} \mu((-\infty, x]) \int_{x}^{m} \frac{1}{\rho_{\mu}}
\end{aligned}
$$

and $B=\max \left(B_{+}, B_{-}\right), D=\max \left(D_{+}, D_{-}\right)$. Then for every smooth function

$$
\int \Phi\left(f^{2}\right) d \mu-\Phi\left(\int f^{2} d \mu\right) \leq\left(144 \gamma B+24\left(1+\frac{M}{\varphi(8)}\right) D\right) \int f^{\prime 2} d \mu .
$$


Proof. The argument is a refinement of the proof of Theorem 26. We explain the points which differ. Without loss of generality we consider a nonnegative function $f$ on $\mathbb{R}$. We consider the associated function $g$ defined by

$$
\begin{array}{llll}
g(x)=f(m)+\int_{m}^{x} f^{\prime}(u) \mathbb{I}_{f^{\prime}(u)>0} d u & \text { if } & & x \geq m \\
g(x)=f(m)+\int_{m}^{x} f^{\prime}(u) \mathbb{I}_{f^{\prime}(u)<0} d u & \text { if } & & x<m .
\end{array}
$$

Set $t=\left(\mu\left(g^{2}\right)\right)^{\frac{1}{2}}$. Then Lemma 24 ensures that

$$
\begin{aligned}
& \int \Phi\left(f^{2}\right) d \mu-\Phi\left(\int f^{2} d \mu\right) \leq \int \Phi_{t^{2}}\left(f^{2}\right) d \mu \\
= & \int_{f^{2} \leq 4 \mu\left(g^{2}\right)} \Phi_{t^{2}}\left(f^{2}\right) d \mu+\int_{f^{2}>4 \mu\left(g^{2}\right)} \Phi_{t^{2}}\left(f^{2}\right) d \mu .
\end{aligned}
$$

For the first term, we use Lemma 25

$$
\begin{aligned}
\int_{f^{2} \leq 4 \mu\left(g^{2}\right)} \Phi_{t^{2}}\left(f^{2}\right) d \mu & =\int_{f \in[0,2 t]} \Phi_{t^{2}}\left(f^{2}\right) \\
& \leq 9 \gamma \int_{f \in[0,2 t]}\left(f-\mu\left(g^{2}\right)^{\frac{1}{2}}\right)^{2} d \mu \leq 9 \gamma \int\left(f-\mu\left(g^{2}\right)^{\frac{1}{2}}\right)^{2} d \mu \\
& \leq 18 \gamma \int(f-g)^{2} d \mu+18 \gamma \int\left(g-\mu\left(g^{2}\right)^{\frac{1}{2}}\right)^{2} d \mu
\end{aligned}
$$

Next observe that

$$
\begin{aligned}
\int(f-g)^{2} d \mu \\
=\int_{m}^{+\infty}\left(\int_{m}^{x}\left[f^{\prime}-f^{\prime} \mathbb{1}_{f^{\prime}>0}\right]\right)^{2} d \mu(x)+\int_{-\infty}^{m}\left(\int_{m}^{x}\left[f^{\prime}-f^{\prime} \mathbb{1}_{f^{\prime}<0}\right]\right)^{2} d \mu(x) \\
\quad=\int_{m}^{+\infty}\left(\int_{m}^{x} f^{\prime} \mathbb{I}_{f^{\prime} \leq 0}\right)^{2} d \mu(x)+\int_{-\infty}^{m}\left(\int_{m}^{x} f^{\prime} \mathbb{I}_{f^{\prime} \geq 0}\right)^{2} d \mu(x) \\
\quad \leq 4 B_{+} \int_{m}^{+\infty} f^{\prime 2} \mathbb{I}_{f^{\prime} \leq 0} d \mu+4 B_{-} \int_{-\infty}^{m} f^{\prime 2} \mathbb{I}_{f^{\prime} \geq 0} d \mu
\end{aligned}
$$

where the last inequality relies on Hardy inequality (see Remark 19). As in the proof of Theorem 26,

$$
\begin{aligned}
\int\left(g-\mu\left(g^{2}\right)^{\frac{1}{2}}\right)^{2} d \mu & \leq 2 C_{P} \int g^{\prime 2} d \mu \\
& =2 C_{P}\left(\int_{m}^{+\infty} f^{\prime 2} \mathbb{1}_{f^{\prime}>0} d \mu+\int_{-\infty}^{m} f^{\prime 2} \mathbb{1}_{f^{\prime}<0} d \mu\right),
\end{aligned}
$$

and we also use the fact that the Poincaré constant $C_{P}$ of $\mu$ satisfies $C_{P} \leq 4 B$. 
Combining the previous three estimates gives

$$
\int_{f^{2} \leq 4 \mu\left(g^{2}\right)} \Phi_{t^{2}}\left(f^{2}\right) d \mu \leq 144 \gamma B \int f^{\prime 2} d \mu .
$$

Now we evaluate the second term in equation (5.10): since $\Phi_{t}(x) \leq x(\varphi(x)-$ $\varphi(t))$ for $x \geq t$

$$
\begin{aligned}
\int_{f^{2}>4 \mu\left(g^{2}\right)} \Phi_{t^{2}}\left(f^{2}\right) d \mu & \leq \int_{f^{2}>4 \mu\left(g^{2}\right)} f^{2}\left(\varphi\left(f^{2}\right)-\varphi\left(\mu\left(g^{2}\right)\right)\right) d \mu \\
& \leq \int_{g^{2}>4 \mu\left(g^{2}\right)} g^{2}\left(\varphi\left(g^{2}\right)-\varphi\left(\mu\left(g^{2}\right)\right)\right) d \mu \\
& \leq \int_{g^{2}>4 \mu\left(g^{2}\right)} g^{2}\left(\varphi\left(\frac{g^{2}}{\mu\left(g^{2}\right)}\right)+M\right) d \mu
\end{aligned}
$$

where we have used $g \geq f \geq 0$ and the fact that $\varphi$ is non-decreasing. At this stage, we apply the decomposition into level sets performed in the proof of Theorem 20 , once on $(m,+\infty)$ and once on $(-\infty, m)$. Note that the function $g$ being non-increasing before $m$ and non-decreasing after, the level sets appearing in the proof are of the form $(-\infty, x], x<m$, and $[x,+\infty), x>m$ for which the $\mu$-capacity is controlled by the hypothesis of the theorem.

The previous two theorems apply to logarithmic Sobolev inequality when $\varphi(x)=\log (x)$, this is how Miclo and Roberto recovered the sufficiency part of the Bobkov-Götze criterion. The next result gives an application to tight versions of Rosen's inequality.

Theorem 28. Let $\beta \in(0,1]$. Let $\mu$ be a probability measure on $\mathbb{R}^{n}$. Assume that one of the following hypotheses holds:

( $i)$ There exists a constant $D$ so that every $A \subset \mathbb{R}^{n}$ with $\mu(A) \leq 1 / 2$ satisfies

$$
\mu(A) \log ^{\beta}\left(1+\frac{2}{\mu(A)}\right) \leq D \operatorname{Cap}_{\mu}(A) .
$$

(ii) The dimension $n=1, \mu$ has density $\rho_{\mu}$. Let $m$ be a median of $\mu$ and

$$
\begin{aligned}
& D_{+}=\sup _{x>m} \mu([x,+\infty)) \log ^{\beta}\left(1+\frac{2}{\mu([x,+\infty))}\right) \int_{m}^{x} \frac{1}{\rho_{\mu}} \\
& D_{-}=\sup _{x<m} \mu((-\infty, x]) \log ^{\beta}\left(1+\frac{2}{\mu((-\infty, x])}\right) \int_{x}^{m} \frac{1}{\rho_{\mu}} .
\end{aligned}
$$

Assume that $D=\max \left(D_{+}, D_{-}\right)$is finite. 
Then for every smooth $f: \mathbb{R}^{n} \rightarrow \mathbb{R}$ one has

$$
\int f^{2} \log ^{\beta}\left(1+f^{2}\right) d \mu-\left(\int f^{2} d \mu\right) \log ^{\beta}\left(1+\int f^{2} d \mu\right) \leq K D \int|\nabla f|^{2} d \mu,
$$

where one can take $K=96$ in case $(i)$ and $K=168$ in case (ii).

Proof. In view of Theorems 26, 27 and Remark 24 all we have to do is to check a few properties of $\Phi_{\beta}(x)=x \varphi_{\beta}(x)$ where $\varphi_{\beta}(x)=\log ^{\beta}(1+x)$. We insist on the more significant ones. The function $\varphi_{\beta}$ is increasing, and since $\beta \leq 1$ it is also concave. From the obvious relation

$$
\log (1+x y) \leq \log ((1+x)(1+y)) \leq \log (1+x)+\log (1+y), \quad x, y>0
$$

and the sub-additivity of $x \mapsto x^{\beta}$ for $\beta \in(0,1]$ we deduce that $\varphi_{\beta}(x y) \leq$ $\varphi_{\beta}(x)+\varphi_{\beta}(y)$. Finally we check the differential properties. Direct calculation gives

$$
x \varphi_{\beta}^{\prime}(x)=\beta x \frac{\log ^{\beta-1}(1+x)}{1+x} \leq \beta\left(\frac{x}{1+x}\right)^{\beta} \leq \beta \leq 1,
$$

where we have used $(1+x) \log (1+x) \geq x$ for $x \geq 0$. Finally, $\Phi_{\beta}$ is concave since

$$
\Phi_{\beta}^{\prime \prime}(x)=\frac{\beta \log ^{\beta-2}(1+x)}{(1+x)^{2}}((2+x) \log (1+x)+(\beta-1) x)
$$

is non-negative due to $(2+x) \log (1+x) \geq(1+x) \log (1+x) \geq x$.

\subsection{A summary}

In Figure 1 we summarize the various implications between the inequalities studied in this section. We hope that it will help the reader to have an overview of the picture.

First remark that thanks to Lemma 19, in Figure 1 , if $T:[0,1] \rightarrow \mathbb{R}_{+}$is non-decreasing and $x \mapsto T(x) / x$ non-increasing, then for $x \geq e$

$$
\frac{1}{3 T(1 / \log x)} \leq \psi(x)=\sup _{p \in(1,2)} \frac{1-x^{\frac{p-2}{p}}}{T(2-p)} \leq \frac{1}{T(1 / \log x)} .
$$

Assumption (H1, see Theorem 20). $F:[0,+\infty) \rightarrow \mathbb{R}$ is a non-decreasing function satisfying $F \equiv 0$ on $[0,2 \rho)$ for some $\rho>1$. Finally $F(x)=\psi(x / \rho)$ for $x \geq 2 \rho$ and $\lambda=1 /(2 \rho)$.

Assumption (H2, see Theorem 21). $F:[0,+\infty) \rightarrow \mathbb{R}$ is a non-decreasing function satisfying $F(1)=0$ and $F$ is $\mathcal{C}^{2}$ on $[0,2 \rho]$. The measure $\mu$ satisfies a Poincaré inequality. Finally $F(x)=\psi(x / \rho)$ for $x \geq 0$ and $\lambda=1 /(2 \rho)$. 


\begin{tabular}{|c|c|c|}
\hline Poincaré inequality & $\Longleftarrow$ & Beckner-type inequality $(T)(5.3)$ \\
\hline \multicolumn{2}{|c|}{$\Uparrow \begin{array}{l}\text { See Remark } 20 \\
\psi(x) \equiv 1, \lambda=\frac{1}{2}\end{array}$} & $\Uparrow \begin{array}{l}\text { See Theorem } 18 \text {. } \\
\psi(x)=\sup _{p \in(1,2)} \frac{1-x^{\frac{p-2}{p}}}{T(2-p)}, \lambda=\frac{1}{2}\end{array}$ \\
\hline \multicolumn{3}{|c|}{$\forall A$ such that $\mu(A) \leq \lambda, \quad \operatorname{Cap}_{\mu}(A) \geq C_{\psi} \mu(A) \psi\left(\frac{1}{\mu(A)}\right)$} \\
\hline
\end{tabular}

$\Downarrow \begin{gathered}\text { Under assumptions } \\ (H 1) \text { or }(H 2) \text { on } F\end{gathered} \quad \Uparrow \begin{gathered}\text { Under assumption } \\ (H 3) \text { on } F\end{gathered}$

Homogenous F-Sobolev inequality (5.1)

Under assumption

$(H 4)$ on $\phi$
$\Downarrow \begin{gathered}\text { Under assumption } \\ (H 5) \text { on } F\end{gathered}$

Poincaré inequality

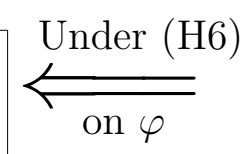

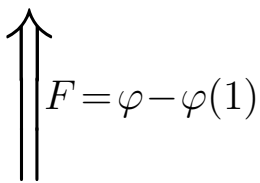

Additive $\varphi$-Sobolev inequality (5.2)

Figure 1: The various implications.

Assumption (H3, see Theorem 22). $F:[0,+\infty) \rightarrow \mathbb{R}$ is a non-decreasing function such that there exists a constant $\gamma>4$ such that for $x \geq 2$, $x \mapsto F(x) / x$ is non-increasing and $F(\gamma x) \leq \gamma F(x) / 4$. Then, $\psi=F$ and $\lambda=1 / 2$.

Assumption (H4, see Theorem 26). The function $\varphi$ is non-decreasing, concave and $\mathcal{C}^{1}$ on $(0,+\infty)$ with $\varphi(8)>0$. Furthermore, there exists two constants $M$ and $\gamma$ such that for any $x, y>0$ one has

$$
x \varphi^{\prime}(x) \leq \gamma \quad \text { and } \quad \varphi(x y) \leq M+\varphi(x)+\phi(y)
$$

The measure $\mu$ satisfies a Poincaré inequality. Finally $\varphi(x)=\psi(x / 2)$ and $\lambda=1 / 4$. 
Assumption (H5, see Lemma 8). $F:[0,+\infty) \rightarrow \mathbb{R}$ is a $\mathcal{C}^{2}$ function on a neighborhood of $1, F(1)=0$ and if $\Phi(x):=x F(x), \Phi^{\prime \prime}(1)>0$.

Assumption (H6). $\varphi:[0,+\infty) \rightarrow \mathbb{R}$ is a $\mathcal{C}^{2}$ function on a neighborhood of 1. Let $\Phi(x):=x \varphi(x)$. The same proof as in Lemma 8 gives that $\mu$ satisfies a Poincaré inequality if $\Phi^{\prime \prime}(1)>0$.

\section{Concentration of measure and generalized Beckner- Latała-Oleszkiewicz inequality}

Recall that a probability measure $\mu$ on $\mathbb{R}^{n}$ satisfies a generalized Beckner inequality if there is a constant $C_{T}$ such that for any smooth function $f$,

$$
\sup _{p \in(1,2)} \frac{\int f^{2} d \mu-\left(\int|f|^{p} d \mu\right)^{\frac{2}{p}}}{T(2-p)} \leq C_{T} \int|\nabla f|^{2} d \mu .
$$

Here $T:[0,1] \rightarrow \mathbb{R}^{+}$is non decreasing, positive on $(0,1]$ and $T(0)=0$. This section explores the concentration results implied by such a property.

Herbst argument, see [28, 39, 3], derives Gaussian concentration for measures $\mu$ satisfying a log-Sobolev inequality along the following lines: let $h$ be a 1-Lipschitz function. Applying the inequality to $\exp (\lambda h / 2)$ provides the next differential inequality for the Laplace transform $H(\lambda)=\int \exp (\lambda h) d \mu$

$$
\lambda H^{\prime}(\lambda)-H(\lambda) \log H(\lambda) \leq \frac{C_{L S}}{4} \lambda^{2} H(\lambda)
$$

Here $C_{L S}$ is the log-Sobolev constant. It can be explicitly solved and gives the sub-Gaussian bound $H(\lambda) \leq \exp \left(\lambda \mu(h)+\left(C_{L S} / 4\right) \lambda^{2}\right)$. This easily yields concentration.

In the case of a Poincaré inequality, this Laplace transform method works [2], but provides an induction inequality for the function $H$. This approach was performed by Latała and Oleszkiewicz for their inequality (i.e. (6.1) with $\left.T(u)=u^{2\left(1-\frac{1}{\alpha}\right)}, 1<\alpha<2\right)$. See [36], where optimization over $p$ is crucial. As also noted in [56], their argument extends as it is to general $T$. It yields

Proposition 29. Let $T:[0,1] \rightarrow \mathbb{R}^{+}$be a non decreasing function such that $T(0)=0$ and positive elsewhere. Define $\theta(x)=1 / T\left(\frac{1}{x}\right)$ for $x \in[1, \infty)$. let $\mu$ be a probability measure on $\mathbb{R}^{n}$ and assume that there exists a constant $C_{T} \geq 0$ such that for any smooth function $f$ satisfies Inequality (6.1). Then any 1-Lipschitz function $h: \mathbb{R}^{n} \rightarrow \mathbb{R}$ verifies $\int|h| d \mu<\infty$, and

(i) for any $t \in[0, \sqrt{T(1)}]$,

$$
\mu\left(\left\{x: h(x)-\mu(h) \geq t \sqrt{C_{T}}\right\}\right) \leq e^{-\frac{t^{2}}{3 T(1)}},
$$


(ii) for any $t \geq \sqrt{T(1)}$,

$$
\mu\left(\left\{x: h(x)-\mu(h) \geq t \sqrt{C_{T}}\right\}\right) \leq e^{-\sqrt{2} \sup _{y \geq 1}\{t \sqrt{\theta(y)}-y\}} .
$$

Proof. We follow the argument of [36]. If $H(\lambda)=\mu\left(e^{\lambda h}\right)$ is the Laplace transform of a 1-Lipschitz function $h$, Inequality (6.1) for $f=\exp (\lambda h / 2)$ gives

$$
H(\lambda)-H\left(\frac{p}{2} \lambda\right)^{2 / p} \leq \frac{C_{T}}{4} T(2-p) \lambda^{2} H(\lambda) .
$$

Then, by induction, we get (see [56]) for any $\lambda<2 / \sqrt{C_{T} T(2-p)}$,

$$
\mu\left(e^{\lambda(h-\mu(h))}\right) \leq\left(1-\frac{C_{T}}{4} T(2-p)\right)^{-2 /(2-p)} .
$$

Chebyshev inequality ensures that for any $p \in[1,2)$ and $\lambda<2 / \sqrt{C_{T} T(2-p)}$,

$$
\mu\left(\left\{x: h(x)-\mu(h) \geq t \sqrt{C_{T}}\right\}\right) \leq e^{-\lambda t \sqrt{C_{T}}}\left(1-\frac{C_{T} \lambda^{2}}{4} T(2-p)\right)^{-\frac{2}{2-p}}
$$

For $t<2 \sqrt{T(1)}$, we set $p=1$ and $\lambda=\frac{t}{T(1) \sqrt{C_{T}}}$ in the latter inequality. We get

$$
\mu\left(\left\{x: h(x)-\mu(h) \geq t \sqrt{C_{T}}\right\}\right) \leq e^{-\frac{t^{2}}{T(1)}}\left(1-\frac{t^{2}}{4 T(1)}\right)^{-2} .
$$

In particular, for $t<\sqrt{T(1)}$ we have $1-\frac{t^{2}}{4 T(1)} \geq e^{-\frac{t^{2}}{3 T(1)}}$. Thus,

$$
\mu\left(\left\{x: h(x)-\mu(h) \geq t \sqrt{C}_{T}\right\}\right) \leq e^{-\frac{t^{2}}{3 T(1)}} .
$$

For the second regime, choose $\lambda$ such that $1-\frac{C_{T} \lambda^{2}}{4} T(2-p)=\frac{1}{2}$. It follows from (6.2) that for any $p \in(1,2)$

$$
\mu\left(\left\{x: h(x)-\mu(h) \geq t \sqrt{C_{T}}\right\}\right) \leq e^{-\frac{\sqrt{2} t}{\sqrt{T(2-p)}}+\frac{2 \ln 2}{2-p}} .
$$

Note that $2 \ln 2 \leq \sqrt{2}$. Thus, if $y:=\frac{1}{2-p}$, we get

$$
\begin{aligned}
-\frac{\sqrt{2} t}{\sqrt{T(2-p)}}+\frac{2 \ln 2}{2-p} & \leq-\sqrt{2}\left\{\frac{t}{\sqrt{T(2-p)}}-\frac{1}{2-p}\right\} \\
& =-\sqrt{2}\{t \sqrt{\theta(y)}-y\} .
\end{aligned}
$$

One concludes the proof by optimizing in $p \in(1,2)$ or equivalently in $y \in$ $(1, \infty)$.

The next statement provides an application of the latter result to concentration with rate $e^{-\Phi(t)}$ for a general convex $\Phi$. When $\Phi(t)=t^{\alpha}, \alpha \in(1,2)$, it reduces to the result by Latała and Oleszkiewicz. 
Corollary 30. Let $\Phi: \mathbb{R}^{+} \rightarrow \mathbb{R}^{+}$be an increasing convex function, with $\Phi(0)=0$. Define $\theta(x)=\left(\Phi^{\prime}\left(\Phi^{-1}(x)\right)\right)^{2}$ for $x \in \mathbb{R}^{+}$and $T(x)=1 / \theta\left(\frac{1}{x}\right)$ for $x \in \mathbb{R}^{+} \backslash\{0\}, T(0)=0$. Here $\Phi^{\prime}$ is the right derivative of $\Phi$. Let $\mu$ be a probability measure on $\mathbb{R}^{n}$ and assume that there exists a constant $C_{T}$ such that it satisfies the generalized Beckner inequality (6.1). Then, for any 1Lipschitz function $h: \mathbb{R}^{n} \rightarrow \mathbb{R}, \int|h|<\infty$ and for any $t \geq \sqrt{T(1)} \vee 2 \Phi^{-1}(1)$,

$$
\mu\left(\left\{x: h(x)-\mu(h) \geq t \sqrt{C_{T}}\right\}\right) \leq e^{-\sqrt{2} \Phi\left(\frac{t}{2}\right)} .
$$

Proof. Thanks to Proposition 29, it is enough to bound $\sup _{y \geq 1}\{t \sqrt{\theta(y)}-y\}$ from below. By assumption $t \geq 2 \Phi^{-1}(1)$, so $\Phi(t / 2) \geq 1$. It follows that for $y=\Phi(t / 2)$,

$$
\sup _{y \geq 1}\{t \sqrt{\theta(y)}-y\} \geq t \sqrt{\theta(\Phi(t / 2))}-\Phi(t / 2)=t \Phi^{\prime}(t / 2)-\Phi(t / 2) .
$$

Since $\Phi$ is convex and $\Phi(0)=0$, one has $x \Phi^{\prime}(x) \geq \Phi(x)$ for all $x \geq 0$. Hence, $\sup _{y \geq 1}\{t \sqrt{\theta(y)}-y\} \geq \Phi(t / 2)$.

Theorem 18 of Section 5 provides a criterion for a measure on the line to satisfy a generalized Beckner inequality. Under mild assumptions, and if one is not interested in estimating the constant, the condition may be further simplified.

Proposition 31. Let $V: \mathbb{R} \rightarrow \mathbb{R}$ be a $\mathcal{C}^{1}$ function. Assume that $d \mu(x)=$ $Z_{V}^{-1} e^{-V(x)} d x$ is a probability measure. Let $T:[0,1] \rightarrow \mathbb{R}^{+}$be non-decreasing with $T(0)=0$ and positive elsewhere. Assume that $x \mapsto T(x) / x$ is nonincreasing. Define $\theta(x)=1 / T(1 / x)$ for $x \in[1, \infty)$. Furthermore, assume that

(i) there exists a constant $A>0$ such that for all $|x| \geq A, V$ is $\mathcal{C}^{2}$ and $\operatorname{sign}(x) V^{\prime}(x)>0$,

(ii) $\lim _{|x| \rightarrow \infty} \frac{V^{\prime \prime}(x)}{V^{\prime}(x)^{2}}=0$,

(iii) $\limsup _{|x| \rightarrow \infty} \frac{\theta\left(V(x)+\log \left|V^{\prime}(x)\right|+\log Z_{V}\right)}{V^{\prime}(x)^{2}}<\infty$.

Then $\mu$ satisfies the following Beckner-type inequality: there exists a constant $C_{T} \geq 0$ such that for any smooth function $f$,

$$
\sup _{p \in(1,2)} \frac{\int f^{2} d \mu-\left(\int|f|^{p} d \mu\right)^{\frac{2}{p}}}{T(2-p)} \leq C_{T} \int f^{\prime 2} d \mu .
$$


Proof. The proof is similar to the one of [12, Proposition 15]. Let $m$ be a median of $\mu$. Under Hypotheses $(i)$ and $(i i)$, when $x$ tends to $\infty$, one has (see e.g. [3, chapter 6])

$$
\int_{m}^{x} e^{V(t)} d t \sim \frac{e^{V(x)}}{V^{\prime}(x)} \quad \text { and } \quad \int_{x}^{\infty} e^{-V(t)} d t \sim \frac{e^{-V(x)}}{V^{\prime}(x)} .
$$

Thus, for $x \geq m$,

$$
\frac{\mu([x, \infty))}{T\left(\frac{1}{\log \left(1+\frac{1}{\mu([x, \infty))}\right)}\right)} \int_{m}^{x} Z_{V} e^{V(t)} d t \sim Z_{V} \frac{\theta\left(V(x)+\log V^{\prime}(x)+\log Z_{V}\right)}{V^{\prime}(x)^{2}} .
$$

By Hypothesis $(i i i)$, this quantity is bounded on $\left[A^{\prime}, \infty\right)$ for some $A^{\prime}$. Since the left hand side is continuous in $x \in\left[m, A^{\prime}\right]$, it is bounded on $(m, \infty)$. It follows from Lemma 19 that the quantity $B_{+}(T)$ defined in Theorem 18 is finite. Similarly $B_{-}(T)<+\infty$. We conclude with Theorem 18 .

The latter results provide a very general condition for dimension free concentration. Starting with an increasing convex concentration rate $\Phi: \mathbb{R}^{+} \rightarrow$ $\mathbb{R}^{+}$with $\Phi(0)=0$, we introduce the function $T(x)=1 /\left(\Phi^{\prime}\left(\Phi^{-1}(x)\right)\right)^{2}$. Under the additional assumption that $\sqrt{\Phi}$ is concave, we know that $T(x) / x$ is non-increasing. Therefore, under the assumptions of Proposition 31, a probability measure $d \mu(x)=Z_{V}^{-1} e^{-V(x)} d x$ on $\mathbb{R}$ satisfies the Beckner inequality with function $T$. By the tensorisation property, the measures $\mu^{\otimes n}$ verify the same inequality and by Corollary 30, they satisfy a dimension free concentration inequality with rate $e^{-\sqrt{2} \Phi(t / 2)}$. Note that our condition about $\sqrt{\Phi}$ is quite natural since, by the Central Limit Theorem, a dimension free concentration inequality has at most a Gaussian rate.

The next application of our criterion provides the best expected concentration rate for certain log-concave distributions.

Corollary 32. Let $\Phi: \mathbb{R}^{+} \rightarrow \mathbb{R}^{+}$be an increasing convex function with $\Phi(0)=0$ and consider the probability measure $d \mu(x)=Z_{\Phi}^{-1} e^{-\Phi(|x|)} d x$. Assume that $\Phi$ is $\mathcal{C}^{2}$ on $\left[\Phi^{-1}(1), \infty\right)$ and that $\sqrt{\Phi}$ is concave.

Then there exits $c>0$ such that for all $n \geq 1$, every 1-Lipschitz function $h: \mathbb{R}^{n} \rightarrow \mathbb{R}$ is $\mu^{\otimes n}$-integrable and satisfies

$$
\mu^{\otimes n}\left(\left\{x: h(x)-\mu^{\otimes n}(h) \geq t \sqrt{c}\right\}\right) \leq e^{-\sqrt{2} \Phi\left(\frac{t}{2}\right)}
$$

provided

$$
t \geq 2 \Phi^{-1}(1) \vee 1 /\left(\Phi^{\prime}\left(\Phi^{-1}(1)\right)\right) .
$$


Proof. Set $\theta(u)=\left(\Phi^{\prime}\left(\Phi^{-1}(u)\right)\right)^{2}$ and $T(u)=1 / \theta(1 / u)$ for $u>0$. The hypotheses on $\Phi$ ensure that $T$ is non-decreasing and $T(u) / u$ is non-increasing. We check below that $\mu$ satisfies a Beckner-type inequality with rate function $T$. By the above argument this implies the claimed concentration inequality for products. Let us check that $V(x)=\Phi(|x|)$ satisfies the three conditions in Proposition 31. By symmetry it is enough to work on $\mathbb{R}^{+}$. Condition $(i)$ is obvious. Condition $(i i)$ is easily checked. Indeed since $\sqrt{\Phi}$ is concave, its second derivative is non-positive when it is defined. So for large $x$ we have $\Phi^{\prime \prime} / \Phi^{\prime 2} \leq 1 /(2 \Phi)$. So $\lim _{+\infty} \Phi=+\infty$ implies that $\lim _{+\infty} \Phi^{\prime \prime} / \Phi^{\prime 2}=0$.

Now we prove that Condition ( $i i i)$ of the latter proposition is verified. Our aim is to bound from above the quantity

$$
K(x):=\frac{\theta\left(\Phi(x)+\log \Phi^{\prime}(x)+\log Z_{\Phi}\right)}{\Phi^{\prime}(x)^{2}} .
$$

By concavity of $\sqrt{\Phi}, \Phi^{\prime 2} / \Phi$ is non-increasing. Thus for $x \geq \Phi^{-1}(1)$, one has $\Phi^{\prime}(x)^{2} \leq \Phi^{\prime}\left(\Phi^{-1}(1)\right)^{2} \Phi(x)$. Hence for $x$ large enough $\log \Phi^{\prime}(x)+\log Z_{\Phi} \leq$ $\Phi(x)$, and $K(x) \leq \theta(2 \Phi(x)) / \Phi^{\prime}(x)^{2}$.

Since $\Phi$ is convex, the slope function $(\Phi(x)-\Phi(0)) / x=\Phi(x) / x$ is nondecreasing. Comparing its values at $x$ and $2 x$ shows that $2 \Phi(x) \leq \Phi(2 x)$. Thus $\theta(2 \Phi(x)) \leq \Phi^{\prime}(2 x)^{2}$ and for $x$ large enough $K(x) \leq \Phi^{\prime}(2 x)^{2} / \Phi^{\prime}(x)^{2}$. As $\Phi^{\prime 2} / \Phi$ is non-increasing we know that $\Phi^{\prime}(2 x)^{2} \leq \frac{\Phi(2 x)}{\Phi(x)} \Phi^{\prime}(x)^{2}$. On the other hand, $\sqrt{\Phi}$ being concave, the slope function $\sqrt{\Phi(x)} / x$ is non-increasing so $\sqrt{\Phi(2 x)} \leq 2 \sqrt{\Phi(x)}$. Finally for $x$ large

$$
K(x) \leq \frac{\Phi^{\prime}(2 x)^{2}}{\Phi^{\prime}(x)^{2}} \leq \frac{\Phi(2 x)}{\Phi(x)} \leq 4 .
$$

The proof is complete.

Remark 26. The hypotheses of Corollary 32 are simple but could be more general. It is plain from Proposition 31 that we need the convexity assumptions only for large values. The argument can be adapted to show that the measures with potential $\Phi(x)=|x|^{\alpha} \log (1+|x|)^{\beta}$ with $1<\alpha<2$ and $\beta \geq 0$ satisfy a dimension free concentration inequality with decay $e^{-C \Phi(t)}$.

Remark 27. Other concentration results for products of log-concave measures on the line follow from Talagrand exponential inequality, see [54, Theorem 2.7.1, Proposition 2.7.4]. They involve a different notion of enlargement depending on the log-concave density itself. However, they imply an analogue of Corollary 32, under the similar assumption that $\Phi(\sqrt{t})$ is subadditive. 


\section{Examples}

In this section we study fundamental examples, starting with $|x|^{\alpha}$ Boltzmann's measures in relation with Beckner's type inequalities. We shall show in particular how to get dimension free inequalities.

\section{1. $|x|^{\alpha}$ Boltzmann's measures}

In this subsection we are looking at the probability measures $d \nu_{\alpha}^{\otimes n}(x)=$ $\prod_{i=1}^{n} Z_{\alpha}^{-1} e^{-2 u_{\alpha}(x)} d x_{i}$ on $\mathbb{R}^{n}$, where as in section $3,1<\alpha<2$ and

$$
u_{\alpha}(x)= \begin{cases}|x|^{\alpha} & \text { for }|x|>1 \\ \frac{\alpha(\alpha-2)}{8} x^{4}+\frac{\alpha(4-\alpha)}{4} x^{2}+\left(1-\frac{3}{4} \alpha+\frac{1}{8} \alpha^{2}\right) & \text { for }|x| \leq 1\end{cases}
$$

We will study two kind of $F$ functionals, starting from the capacity-measure point of view. For each of them we give functional inequalities and derive hypercontractivity (or hyperboundedness) property satisfied by the semigroup.

The first function of interest for us is

$$
\begin{aligned}
F_{\alpha}: \mathbb{R}^{+} & \rightarrow \mathbb{R} \\
x & \mapsto(\log (1+x))^{2\left(1-\frac{1}{\alpha}\right)}-(\log 2)^{2\left(1-\frac{1}{\alpha}\right)} .
\end{aligned}
$$

Note that it is a $\mathcal{C}^{2}$ non-decreasing function satisfying $F_{\alpha}(1)=0$. It is negative for $x<1$ and positive for $x>1$.

The second function of interest is

$$
\begin{aligned}
\widetilde{F}_{\alpha}: \mathbb{R}^{+} & \rightarrow \mathbb{R} \\
x & \mapsto\left\{\begin{array}{lll}
0 & \text { if } & x \in[0,2 \rho] \\
(\log (x))^{2\left(1-\frac{1}{\alpha}\right)}-(\log 2 \rho)^{2\left(1-\frac{1}{\alpha}\right)} & \text { if } & x \geq 2 \rho
\end{array},\right.
\end{aligned}
$$

where $\rho>1$ is a fixed parameter. Note that $\widetilde{F}_{\alpha}$ is continuous but not $\mathcal{C}^{2}$. On the other hand, it is always non-negative.

Proposition 33. Let $1<\alpha<2$. Let $F_{\alpha}$ and $\widetilde{F}_{\alpha}$ defined in (7.2) and (7.3) respectively. Denote by $\nu_{\alpha}^{\otimes n}=\otimes_{i=1}^{n} \nu_{\alpha, i}$ the product measure of $n$ copies of $d \nu_{\alpha}(x)=Z_{\alpha}^{-1} e^{-2 u_{\alpha}(x)} d x$.

Then, there exist two constants $C=C(\alpha)$ and $\widetilde{C}=\widetilde{C}(\alpha, \rho)$ such that for any integer $n$, for any smooth enough function $f: \mathbb{R}^{n} \rightarrow \mathbb{R}$,

$$
\int f^{2} F_{\alpha}\left(\frac{f^{2}}{\nu_{\alpha}^{\otimes n}\left(f^{2}\right)}\right) d \nu_{\alpha}^{\otimes n} \leq C \int|\nabla f|^{2} d \nu_{\alpha}^{\otimes n},
$$

and

$$
\int f^{2} \widetilde{F}_{\alpha}\left(\frac{f^{2}}{\nu_{\alpha}^{\otimes n}\left(f^{2}\right)}\right) d \nu_{\alpha}^{\otimes n} \leq \widetilde{C} \int|\nabla f|^{2} d \nu_{\alpha}^{\otimes n} .
$$


Proof. We start with $F_{\alpha}$. Fix $n=1$. Then 0 is a median of $\nu_{\alpha}$. When $x$ tends to infinity, it is easy to check that

$$
\int_{0}^{x} e^{2 u_{\alpha}(t)} d t \sim \frac{e^{2 x^{\alpha}}}{2 \alpha x^{\alpha-1}} \quad \text { and } \quad \int_{x}^{\infty} e^{-2 u_{\alpha}(t)} d t \sim \frac{e^{-2 x^{\alpha}}}{2 \alpha x^{\alpha-1}} .
$$

It follows that the two constants $D_{+}$and $D_{-}$introduced in Theorem 28 with $\beta=2\left(1-\frac{1}{\alpha}\right)$ are finite. Then, we conclude by Theorem 28 that there exists a constant $C_{\alpha}$ such that for every function $f$ on $\mathbb{R}$,

$$
\begin{aligned}
& \int f^{2} \log ^{\beta}\left(1+f^{2}\right) d \nu_{\alpha}-\left(\int f^{2} d \nu_{\alpha}\right) \log ^{\beta}\left(1+\int f^{2} d \nu_{\alpha}\right) \\
& \leq C_{\alpha} \int|\nabla f|^{2} d \nu_{\alpha}
\end{aligned}
$$

Then, for any integer $n$, by Lemma 12 the latter inequality holds for $\nu_{\alpha}^{\otimes n}$ in $\mathbb{R}^{n}$. Finally, applying the inequality to $f^{2} / \nu_{\alpha}^{\otimes n}\left(f^{2}\right)$ gives the expected result.

The case of $\widetilde{F}_{\alpha}$ is a bit more difficult. Let $\beta=2\left(1-\frac{1}{\alpha}\right)$ and $T(x)=|x|^{\beta}$. It is easy to check that the hypotheses of Proposition 31 are satisfied (for $\left.\Phi=2 u_{\alpha}\right)$ and thus that there exists a constant $\widetilde{C}=\widetilde{C}(\alpha)$ such that for any function $f: \mathbb{R} \rightarrow \mathbb{R}$,

$$
\sup _{p \in(1,2)} \frac{\int f^{2} d \nu_{\alpha}-\left(\int|f|^{p} d \nu_{\alpha}\right)^{\frac{2}{p}}}{(2-p)^{\beta}} \leq \widetilde{C} \int|\nabla f|^{2} d \nu_{\alpha} .
$$

Now, by tensorisation property (see [36]), the same inequality holds for $\nu_{\alpha}^{\otimes n}$ with the same constant $\widetilde{C}$ (independent of $n$ ). Thus, by Theorem 18 together with Lemma 19 (recall that $T(x)=|x|^{\beta}$ ), it follows that for any integer $n$, any Borel set $A \subset \mathbb{R}^{n}$ with $\nu_{\alpha}^{\otimes n}(A) \leq 1 / 2$,

$$
\nu_{\alpha}^{\otimes n}(A)\left(\log \left(1+\frac{1}{\nu_{\alpha}^{\otimes n}(A)}\right)\right)^{\beta} \leq 2 \widetilde{C} \operatorname{Cap}_{\nu_{\alpha}^{\otimes n}}(A) .
$$

Now, for any $x \geq 2 \rho, \widetilde{F}_{\alpha}(\rho x) \leq(\log (1+x))^{\beta}$. Therefore, for any Borel set $A \subset \mathbb{R}^{n}$ with $\nu_{\alpha}^{\otimes n}(A) \leq 1 /(2 \rho)$,

$$
\nu_{\alpha}^{\otimes n}(A) \widetilde{F}_{\alpha}\left(\frac{\rho}{\nu_{\alpha}^{\otimes n}(A)}\right) \leq 2 \widetilde{C} \operatorname{Cap}_{\nu_{\alpha}^{\otimes n}}(A) .
$$

The expected result follows from Theorem 20. This achieves the proof.

Remark 28. It is not difficult to check that

$$
0<\inf _{\alpha \in(1,2)} C(\alpha)<\sup _{\alpha \in(1,2)} C(\alpha)<+\infty .
$$

This means that the constant $C(\alpha)$ appearing in Proposition 33 can be chosen independently of $\alpha \in(1,2)$. This uniformity will be useful for applications. 
Corollary 34. Let $1<\alpha<2$. Let $F_{\alpha}$ defined in (7.2). Denote by $\nu_{\alpha}^{\otimes n}=$ $\otimes_{i=1}^{n} \nu_{\alpha, i}$ the product measure of $n$ copies of the probability measure $d \nu_{\alpha}(x)=$ $Z_{\alpha}^{-1} e^{-2 u_{\alpha}(x)} d x$. Define for any $q \geq 0$, any $x \geq 0, \tau_{q}^{(\alpha)}(x)=x^{2} e^{q F_{\alpha}\left(x^{2}\right)}$.

Then, there exists a universal constant $C$ such that for any integer $n$, any function $f: \mathbb{R}^{n} \rightarrow \mathbb{R}$ and any $t \geq 0$,

$$
N_{\tau_{q(t)}^{(\alpha)}}\left(\mathbf{P}_{t} f\right) \leq e^{\frac{C}{2} t}\|f\|_{2}
$$

where $q(t)=C t$ and $N_{\Phi}(g):=\inf \left\{\lambda: \int \Phi(g / \lambda) d \nu_{\alpha}^{\otimes n} \leq 1\right\}$.

Proof. The result is a direct consequence of Theorem 6, using Proposition 33 and Lemma 35 below.

Lemma 35. Let $1<\alpha<2$. Let $F_{\alpha}$ defined in (7.2). Define for any $q \geq 0$, any $x \geq 0, \tau_{q}^{(\alpha)}(x)=x^{2} e^{q F_{\alpha}\left(x^{2}\right)}$. Then,

(i) For any $x \geq 0$, any $q \geq 0$,

$$
\left(\tau_{q}^{(\alpha)}\right)^{\prime \prime} \tau_{q}^{(\alpha)} \geq \frac{5-(4 / \alpha)}{4}\left(\tau_{q}^{(\alpha)}\right)^{\prime 2} \geq \frac{1}{4}\left(\tau_{q}^{(\alpha)}\right)^{\prime 2}
$$

(ii) for any $x \geq 0$, any $q \geq 0$,

$$
\tau_{q}^{(\alpha)}(x) F\left(x^{2}\right) \leq \tau_{q}^{(\alpha)}(x) F\left(\tau_{q}^{(\alpha)}(x)\right)+1
$$

Proof. Let $\beta=2\left(1-\frac{1}{\alpha}\right)$. Then $0<\beta<1$. It is easy to check that for any $x>0$,

$$
-\frac{x F_{\alpha}^{\prime \prime}(x)}{F_{\alpha}^{\prime}(x)}=\frac{x(1-\beta+\log (1+x))}{(1+x) \log (1+x)} \leq 2-\beta .
$$

We conclude the proof of point $(i)$ applying Proposition 7 (note that $2+\frac{1}{2}-$ $\left.\frac{5-(4 / \alpha)}{2}=2-\beta\right)$.

Note that $m_{F_{\alpha}}:=\left|\min _{x \in(0,1)} x F_{\alpha}(x)\right| \leq 1$. Hence, using remark 10 concludes the proof of point $(i i)$.

The analogue of Corollary 34 for $\widetilde{F}_{\alpha}$ is a bit harder due to differentiation problem at $x=2 \rho$. The result is the following:

Corollary 36. Let $1<\alpha<2$. Let $\widetilde{F}_{\alpha}$ defined in (7.3). Denote by $\nu_{\alpha}^{\otimes n}=$ $\otimes_{i=1}^{n} \nu_{\alpha, i}$ the product measure of $n$ copies of the probability measure $d \nu_{\alpha}(x)=$ $Z_{\alpha}^{-1} e^{-2 u_{\alpha}(x)} d x$. Define for any $q \geq 0$, any $x \geq 0, \widetilde{\tau}_{q}^{(\alpha)}(x)=x^{2} e^{q \widetilde{F}_{\alpha}\left(x^{2}\right)}$.

Then, there exists a constant $\widetilde{C}=\widetilde{C}(\alpha, \rho)$ such that for any integer $n$, any function $f: \mathbb{R}^{n} \rightarrow \mathbb{R}$ and any $t \geq 0$,

$$
N_{\widetilde{\tau}_{q(t)}^{(\alpha)}}\left(\mathbf{P}_{t} f\right) \leq\|f\|_{2}
$$

where $q(t)=\widetilde{C} t$ and $N_{\Phi}(g):=\inf \left\{\lambda: \int \Phi(g / \lambda) d \nu_{\alpha}^{\otimes n} \leq 1\right\}$. 
Proof. Let $g$ be a $\mathcal{C}^{\infty}$ non-negative function with compact support in $[-1,0]$ and such that $\int g(y) d y=1$. For any $\varepsilon>0$ define $g_{\varepsilon}(x)=\frac{1}{\varepsilon} g\left(\frac{x}{\varepsilon}\right)$ and note that $\widetilde{F}_{\alpha} * g_{\varepsilon}(x):=\int \widetilde{F}_{\alpha}(x-y) g_{\varepsilon}(y) d y$ is a $\mathcal{C}^{\infty}$ function.

Define for any $\varepsilon>0$, any $q \geq 0, \widetilde{\tau}_{q, \varepsilon}^{(\alpha)}(x)=x^{2} e^{q \widetilde{F}_{\alpha} * g_{\varepsilon}\left(x^{2}\right)}$.

Thanks to Lemma 37 below, $\widetilde{F}_{\alpha} * g_{\varepsilon}$ satisfies the hypothesis of Theorem 6 , uniformly in $n$. Thus, by Theorem 6 there exists two constants $\widetilde{C}=\widetilde{C}(\alpha, \rho)$ and $\widetilde{C}^{\prime}=\widetilde{C}^{\prime}(\alpha, \rho)$ (maybe different from those one of Lemma 37$)$ such that for any integer $n$, any function $f: \mathbb{R}^{n} \rightarrow \mathbb{R}$ and any $t \geq 0$,

$$
N_{\widetilde{\tau}_{q(t), \varepsilon}^{(\alpha)}}\left(\mathbf{P}_{t} f\right) \leq e^{\frac{1}{2}\left(\widetilde{F}_{\alpha}(2 \rho+\varepsilon)+\varepsilon \widetilde{C}^{\prime}\right) t}\|f\|_{2} .
$$

Then, it is easy to verify that for any function $f$, any $t$, when $\varepsilon$ tends to 0 ,

$$
N_{\widetilde{\tau}_{q(t), \varepsilon}^{(\alpha)}}\left(\mathbf{P}_{t} f\right) \rightarrow N_{\widetilde{\tau}_{q(t)}^{(\alpha)}}\left(\mathbf{P}_{t} f\right) \quad \text { and } \quad e^{\frac{1}{2}\left(\widetilde{F}_{\alpha}(2 \rho+\varepsilon)+\varepsilon \widetilde{C}^{\prime}\right) t} \rightarrow 1 .
$$

This achieves the proof.

Lemma 37. Let $1<\alpha<2$. Let $\widetilde{F}_{\alpha}$ defined in (7.3). Denote by $\nu_{\alpha}^{\otimes n}=$ $\otimes_{i=1}^{n} \nu_{\alpha, i}$ the product measure of $n$ copies of $d \nu_{\alpha}(x)=Z_{\alpha}^{-1} e^{-2 u_{\alpha}(x)} d x$. Define for any $q \geq 0$, any $x \geq 0, \widetilde{\tau}_{q}^{(\alpha)}(x)=x^{2} e^{q \widetilde{F}_{\alpha}\left(x^{2}\right)}$.

Let $g$ be a $\mathcal{C}^{\infty}$ non-negative function with compact support in $[-1,0]$ and such that $\int g(y) d y=1$. Define $g_{\varepsilon}(x)=\frac{1}{\varepsilon} g\left(\frac{x}{\varepsilon}\right)$, and $\widetilde{F}_{\alpha} * g_{\varepsilon}(x):=$ $\int \widetilde{F}_{\alpha}(x-y) g_{\varepsilon}(y) d y$ for any $\varepsilon>0$, and for any $q \geq 0, \widetilde{\tau}_{q, \varepsilon}^{(\alpha)}(x)=x^{2} e^{q \widetilde{F}_{\alpha} * g_{\varepsilon}\left(x^{2}\right)}$. Then,

(i) for any $\varepsilon>0$ and any $q \geq 0$,

$$
\left(\widetilde{\tau}_{q, \varepsilon}^{(\alpha)}\right)^{\prime \prime} \widetilde{\tau}_{q, \varepsilon}^{(\alpha)} \geq \frac{3-2(2-\alpha) /(\alpha \log (2 \rho))}{4}\left(\widetilde{\tau}_{q, \varepsilon}^{(\alpha)}\right)^{\prime 2}
$$

(ii) For any $\varepsilon>0$ small enough, any $q \geq 0$, and any $x \geq 0$,

$$
\widetilde{F}_{\alpha} * g_{\varepsilon}\left(x^{2}\right) \leq \widetilde{F}_{\alpha} * g_{\varepsilon}\left(\widetilde{\tau}_{q, \varepsilon}^{(\alpha)}(x)\right)
$$

(iii) There exist two constants $\widetilde{C}=\widetilde{C}(\alpha, \rho)$ and $\widetilde{C}^{\prime}=\widetilde{C}^{\prime}(\alpha, \rho)$ such that for any integer $n$, any function $f: \mathbb{R}^{n} \rightarrow \mathbb{R}$ and any $\varepsilon>0$ small enough,

$$
\begin{aligned}
\int f^{2} \widetilde{F}_{\alpha} * g_{\varepsilon}\left(\frac{f^{2}}{\nu_{\alpha}^{\otimes n}\left(f^{2}\right)}\right) d \nu_{\alpha}^{\otimes n} \leq & \widetilde{C} \int|\nabla f|^{2} d \nu_{\alpha}^{\otimes n} \\
& +\left(\widetilde{F}_{\alpha}(2 \rho+\varepsilon)+\varepsilon \widetilde{C}^{\prime}\right) \int f^{2} d \nu_{\alpha}^{\otimes n} .
\end{aligned}
$$


Proof. Let $\beta=2\left(1-\frac{1}{\alpha}\right)$.

We start with $(i)$. The result is obviously true for $x \leq 2 \rho$. For $x>2 \rho$, an easy computation gives

$$
-\frac{x \widetilde{F}_{\alpha}^{\prime \prime}(x)}{\widetilde{F}_{\alpha}^{\prime}(x)}=\frac{1-\beta+\log x}{\log x} \leq 1+\frac{1-\beta}{\log (2 \rho)}=1+\frac{2-\alpha}{\alpha \log 2 \rho} .
$$

Thus, by Lemma 38 below we get that for any $\varepsilon>0$, any $x \geq 0$,

$$
x\left(\widetilde{F}_{\alpha} * g_{\varepsilon}\right)^{\prime \prime}(x)+\left(1+\frac{2-\alpha}{\alpha \log 2 \rho}\right)\left(\widetilde{F}_{\alpha} * g_{\varepsilon}\right)^{\prime}(x) \geq 0 .
$$

The result follows from Proposition 7.

For $(i i)$ note that for any $\varepsilon \leq 2 \rho-1, \widetilde{F}_{\alpha} * g_{\varepsilon} \equiv 0$ on $[0,1]$. Thus the result becomes obvious thanks to Remark 12 .

Next we deal with $($ iii $)$. First note that $\widetilde{F}_{\alpha} * g_{\varepsilon} \equiv 0$ on $[0,2 \rho-\varepsilon]$. Then, for $x \in[2 \rho-\varepsilon, 2 \rho]$, since $\widetilde{F}_{\alpha}$ is non-decreasing,

$$
\widetilde{F}_{\alpha} * g_{\varepsilon}(x)=\int_{\{-\varepsilon \leq y \leq 0\}} \widetilde{F}_{\alpha}(x-y) g_{\varepsilon}(y) d y \leq \widetilde{F}_{\alpha}(2 \rho+\varepsilon) .
$$

Finally, for $x>2 \rho$, since $\widetilde{F}_{\alpha}^{\prime}$ is non-increasing,

$$
\begin{aligned}
\widetilde{F}_{\alpha} * g_{\varepsilon}(x) & =\widetilde{F}_{\alpha}(x)+\int_{\{-\varepsilon \leq y \leq 0\}}\left(\widetilde{F}_{\alpha}(x-y)-\widetilde{F}_{\alpha}(x)\right) g_{\varepsilon}(y) d y \\
& \leq \widetilde{F}_{\alpha}(x)+\varepsilon \max _{\{x \leq z \leq x+\varepsilon\}} \widetilde{F}_{\alpha}^{\prime}(z) \\
& \leq \widetilde{F}_{\alpha}(x)+\varepsilon \widetilde{F}_{\alpha}^{\prime}\left(2 \rho^{+}\right),
\end{aligned}
$$

where we have set

$$
\widetilde{F}_{\alpha}^{\prime}\left(2 \rho^{+}\right):=\lim _{x \rightarrow 2 \rho^{+}} F_{\alpha}^{\prime}(x)=\frac{\alpha-1}{\alpha \rho}(\log 2 \rho)^{\frac{\alpha-2}{\alpha}} .
$$

Hence, for any integer $n$, for any function $f: \mathbb{R}^{n} \rightarrow \mathbb{R}$ and any $\varepsilon>0$ small enough,

$$
\begin{aligned}
\int f^{2} \widetilde{F}_{\alpha} * g_{\varepsilon}\left(\frac{f^{2}}{\nu_{\alpha}^{\otimes n}\left(f^{2}\right)}\right) d \nu_{\alpha}^{\otimes n} \leq & \int f^{2} \widetilde{F}_{\alpha}\left(\frac{f^{2}}{\nu_{\alpha}^{\otimes n}\left(f^{2}\right)}\right) d \nu_{\alpha}^{\otimes n} \\
& +\left(\widetilde{F}_{\alpha}(2 \rho+\varepsilon)+\varepsilon \widetilde{F}_{\alpha}^{\prime}\left(2 \rho^{+}\right)\right) \int f^{2} d \nu_{\alpha}^{\otimes n} .
\end{aligned}
$$

The claimed result follows from Proposition 33, with $\widetilde{C}^{\prime}=\frac{\alpha-1}{\alpha \rho}(\log 2 \rho)^{\frac{\alpha-2}{\alpha}}$. 
Lemma 38. Let $F: \mathbb{R}+\rightarrow \mathbb{R}^{+}$be a continuous non-decreasing function such that $F \equiv 0$ on $[0,2 \rho]$, for some $\rho>1$, and $F>0$ on $(2 \rho, \infty)$. Assume that $F$ is $\mathcal{C}^{2}$ on $(2 \rho, \infty)$ and that $\lim _{x \rightarrow 2 \rho^{+}} F^{\prime}(x)$ and $\lim _{x \rightarrow 2 \rho^{+}} F^{\prime \prime}(x)$ exist. Furthermore, assume that $F^{\prime \prime} \leq 0$ on $(2 \rho, \infty)$.

Let $g$ be a $\mathcal{C}^{\infty}$ non-negative function with compact support in $[-1,0]$ and such that $\int g(y) d y=1$. Define $g_{\varepsilon}(x)=\frac{1}{\varepsilon} g\left(\frac{x}{\varepsilon}\right)$ for any $\varepsilon>0$.

Assume that for some $\lambda>0, F$ satisfies for all $x \neq 2 \rho$

$$
x F^{\prime \prime}(x)+\lambda F^{\prime}(x) \geq 0 .
$$

Then, for any $\varepsilon>0$ small enough, any $x \geq 0$,

$$
x\left(F * g_{\varepsilon}\right)^{\prime \prime}(x)+\lambda\left(F * g_{\varepsilon}\right)^{\prime}(x) \geq 0 .
$$

Here, $F * g_{\varepsilon}(x):=\int F(x-y) g_{\varepsilon}(y) d y$.

Proof. Note that for any $\varepsilon>0, F * g_{\varepsilon}$ is a $\mathcal{C}^{\infty}$ function. Fix $\varepsilon \in(0,2 \rho)$.

If $x \in(0,2 \rho-\varepsilon)$, then it is easy to check that $F * g_{\varepsilon}(x)=0$. Thus (7.5) holds for any $x \in(0,2 \rho-\varepsilon)$ and by continuity for any $x \in[0,2 \rho-\varepsilon)$.

Now fix $x \in(2 \rho, \infty)$ and note that for any $y \in \operatorname{supp}\left(g_{\varepsilon}\right) \subset[-\varepsilon, 0]$, $x-y>2 \rho$. Thus $F^{\prime}(x-y)$ and $F^{\prime \prime}(x-y)$ are well defined. It follows that

$$
x\left(F * g_{\varepsilon}\right)^{\prime \prime}(x)+\lambda\left(F * g_{\varepsilon}\right)^{\prime}(x)=\int\left[x F^{\prime \prime}(x-y)+\lambda F^{\prime}(x-y)\right] g_{\varepsilon}(y) d y .
$$

Since $F^{\prime \prime} \leq 0$ and $y \leq 0, x F^{\prime \prime}(x-y) \geq(x-y) F^{\prime \prime}(x-y)$. Hence, the left hand side of the latter inequality is bounded below by

$$
\int\left[(x-y) F^{\prime \prime}(x-y)+\lambda F^{\prime}(x-y)\right] g_{\varepsilon}(y) d y \geq 0
$$

by our assumption on $F$. Thus (7.5) holds for any $x>2 \rho$ and it remains the case $x \in[2 \rho-\varepsilon, 2 \rho]$. By continuity, it is enough to deal with $x \in(2 \rho-\varepsilon, 2 \rho)$.

Fix $x \in(2 \rho-\varepsilon, 2 \rho)$. Choose $h$ such that $x+h<2 \rho$ and note that if $x-y \leq 2 \rho$, then $F(x-y)=0$. Hence,

$$
\begin{aligned}
& \int \frac{F(x-y+h)-F(x-y)}{h} g_{\varepsilon}(y) d y= \\
& \int_{-\varepsilon \leq y<-(2 \rho-x)} \frac{F(x-y+h)-F(x-y)}{h} g_{\varepsilon}(y) d y+\int_{-(2 \rho-x) \leq y \leq 0} \frac{F(x-y+h)}{h} g_{\varepsilon}(y) d y .
\end{aligned}
$$

The second term in the latter equality is non-negative because $F$ is nonnegative. It follows by Lebesgue Theorem that

$$
\left(F * g_{\varepsilon}\right)^{\prime}(x) \geq \int_{\{-\varepsilon \leq y<-(2 \rho-x)\}} F^{\prime}(x-y) g_{\varepsilon}(y) d y .
$$


The same holds for $\left(F * g_{\varepsilon}\right)^{\prime \prime}(x)$ because $F^{\prime}$ is non-negative. Now, as in the previous argument, by our hypothesis on $F, x\left(F * g_{\varepsilon}\right)^{\prime \prime}(x)+\lambda\left(F * g_{\varepsilon}\right)^{\prime}(x)$ is bounded below by

$$
\begin{aligned}
& \int_{\{-\varepsilon \leq y<-(2 \rho-x)\}}\left[x F^{\prime \prime}(x-y)+\lambda F^{\prime}(x-y)\right] g_{\varepsilon}(y) d y \\
& \quad \geq \int_{\{-\varepsilon \leq y<-(2 \rho-x)\}}\left[(x-y) F^{\prime \prime}(x-y)+\lambda F^{\prime}(x-y)\right] g_{\varepsilon}(y) d y \geq 0 .
\end{aligned}
$$

\subsection{A general perturbation argument}

In Section 3 we discussed a perturbation argument in order to prove the hyperboundedness of $\mathbf{P}_{t}^{(\alpha)}$ the semi group associated to $\nu_{\alpha}$. In the previous subsection we recovered and improved these results by using the capacitymeasure approach and the Gross-Orlicz theory. We shall below show that one can also derive the results in Proposition 33 by a perturbation argument on $F_{\alpha}$-Sobolev inequalities (see [21, section 4] for a similar argument for usual log-Sobolev inequalities). The argument can be easily generalized to other situations, but we shall not develop a complete perturbation theory here.

Recall that Lebesgue measure on $\mathbb{R}^{n}$ satisfies a family of $F=\log _{+}$ Sobolev inequalities i.e. for all $\eta>0$ and all $f$ belonging to $\mathbb{L}^{1}(d x) \cap \mathbb{L}^{\infty}(d x)$ such that $\int f^{2} d x=1$

$$
\int f^{2} \log _{+} f^{2} d x \leq 2 \eta \int|\nabla f|^{2} d x+2+\frac{n}{2} \log \left(\frac{1}{\pi \eta}\right),
$$

see e.g. [27] Theorem 2.2.4. In the sequel we denote by $c(\eta)$ the constant in (7.6).

Set $\beta=2\left(1-\frac{1}{\alpha}\right)$ which is less than 1 . According to Lemma 47 in the next section $F_{\alpha}(x)=\log ^{\beta}(1+x)-\log ^{\beta}(2) \leq \log x$ for $x \geq 1$. Since $F_{\alpha}(x)$ is non positive for $x \leq 1$, it follows

$$
\int f^{2} F_{\alpha}\left(f^{2}\right) d x \leq \int f^{2} \log _{+} f^{2} d x .
$$

Let $V$ be smooth and satisfying the conditions stated in Section 3. Denote by $\nu_{V}(d x)=e^{-2 V} d x$ the associated Boltzmann measure and introduce $g=e^{V} f$. Remark that $\int g^{2} d \nu_{V}=1$. According to (7.6) and (7.7), a simple calculation yields

$$
\begin{aligned}
\int g^{2} F_{\alpha}\left(g^{2} e^{-2 V}\right) d \nu_{V} \leq & 2 \eta \int|\nabla g|^{2} d \nu_{V}+c(\eta) \\
& +2 \eta \int g^{2}\left(\Delta V-|\nabla V|^{2}\right) d \nu_{V}
\end{aligned}
$$


Since $\beta<1,(A+B)^{\beta} \leq A^{\beta}+B^{\beta}$ for positive $A$ and $B$. Hence if $V \geq 0$

$$
\log ^{\beta}\left(1+g^{2} e^{-2 V}\right)+\log ^{\beta}\left(e^{2 V}\right) \geq \log ^{\beta}\left(e^{2 V}+g^{2}\right) \geq \log ^{\beta}\left(1+g^{2}\right),
$$

while for $V \leq 0$

$$
\log ^{\beta}\left(1+g^{2} e^{-2 V}\right)+\log ^{\beta}\left(e^{2|V|}\right) \geq \log ^{\beta}\left(1+g^{2} e^{-2 V}\right) \geq \log ^{\beta}\left(1+g^{2}\right) .
$$

It follows that

$$
\begin{aligned}
\int g^{2} F_{\alpha}\left(g^{2}\right) d \nu_{V} \leq & 2 \eta \int|\nabla g|^{2} d \nu_{V}+c(\eta) \\
& +\int g^{2}\left(\log ^{\beta}\left(e^{2|V|}\right)+2 \eta\left(\Delta V-|\nabla V|^{2}\right)\right) d \nu_{V}
\end{aligned}
$$

Now we introduce the convex conjugate function $H_{\alpha}$ of $x \mapsto x F_{\alpha}(x)$. Using the Young's inequality

$$
x y \leq \varepsilon x F_{\alpha}(x)+H_{\alpha}(y / \varepsilon)
$$

in (7.9) we obtain

$$
\begin{aligned}
\int g^{2} F_{\alpha}\left(g^{2}\right) d \nu_{V} & \leq \frac{2 \eta}{1-\varepsilon} \int|\nabla g|^{2} d \nu_{V}+c(\eta, \varepsilon) \\
+\frac{1}{1-\varepsilon} & \int H_{\alpha}\left((1 / \varepsilon)\left((2|V|)^{\beta}+2 \eta\left(\Delta V-|\nabla V|^{2}\right)\right)\right) d \nu_{V}
\end{aligned}
$$

We have thus obtained

Theorem 39. Let $\nu_{V}(d x)=e^{-2 V} d x$ be a Boltzmann measure defined for a smooth $V$ as in Section 3. Denote by $H_{\alpha}$ the convex conjugate of $x \mapsto x F_{\alpha}(x)$. Assume that

(i) there exist some $\xi>0$ and some $\lambda>0$ such that

$$
\int H_{\alpha}\left([(2+\xi)|V|]^{\beta}+\lambda\left[\Delta V-|\nabla V|^{2}\right]\right) d \nu_{V}<+\infty
$$

(ii) $\nu_{V}$ satisfies a Poincaré inequality.

Then the conclusions of Proposition 33 for $F_{\alpha}$ are still true replacing $\nu_{\alpha}$ by $\nu_{V}$. As a consequence the conclusions of Corollary 34 are also still true.

Both conditions ( $i$ ) and (ii) are satisfied when $V$ satisfies assumption $O B$ in Section 3 with $G(y)=c|y|^{2\left(1-\frac{1}{\alpha}\right)}$ for some $c$ and $V$ goes to infinity at infinity. 
Proof. Inequality (7.10) and hypothesis $(i)$ ensure that $\nu_{V}$ satisfies a defective homogeneous $F_{\alpha}$ Sobolev inequality. It is easily seen that $F_{\alpha}$ fulfills the hypotheses of the Rothaus-Orlicz Lemma 9. Hence $(i i)$ and Theorem 10 allow to tight the homogeneous $F_{\alpha}$-Sobolev inequality. Since

$$
\log ^{\beta}\left(1+g^{2}\right) \leq \log ^{\beta}\left(1+\frac{g^{2}}{\int g^{2}}\right)+\log ^{\beta}\left(1+\int g^{2}\right)
$$

Inequality (7.4) holds when we replace $\nu_{\alpha}$ by $\nu_{V}$. Hence we may use the tensorisation property as in Proposition 33 to end the proof.

Finally $(i)$ is clearly implied by OB, while $(i i)$ follows from Remark 4 .

Again the situation is more delicate when dealing with $\widetilde{F}_{\alpha}$.

\section{Isoperimetric inequalities}

In this section we show that the Orlicz-hypercontractivity property implies isoperimetric inequalities. These results are more precise than the concentration inequalities derived in Section 6 via the Beckner type inequalities. Let us recall the basic definitions. Let $\mu$ be a Borel measure on $\mathbb{R}^{n}$. For a measurable set $A \subset \mathbb{R}^{n}$ we define its $\mu$-boundary measure as

$$
\mu_{s}(\partial A)=\liminf _{h \rightarrow 0^{+}} \frac{\mu\left(A_{h}\right)-\mu(A)}{h},
$$

where $A_{h}=\left\{x \in \mathbb{R}^{n}, d(x, A) \leq h\right\}=A+h B_{2}^{n}$ is the $h$-enlargement of $A$ in the Euclidean distance (here $B_{2}^{n}=\left\{x \in \mathbb{R}^{n} ;|x| \leq 1\right\}$ ). The isoperimetric function (or profile) of a probability measure on $\mathbb{R}^{n}$ is

$$
I_{\mu}(a)=\inf \left\{\mu_{s}(\partial A) ; \mu(A)=a\right\}, \quad a \in[0,1] .
$$

We shall write $I_{\mu^{k}}$ for the isoperimetric function of the product measure (on $\mathbb{R}^{n k}$ the enlargements are for the Euclidean distance, that is the $\ell_{2}$ combination of the distances on the factors). Finally we set $I_{\mu^{\infty}}:=\inf _{k \geq 1} I_{\mu^{k}}$.

We follow Ledoux's approach of an inequality by Buser [37] bounding from below the Cheeger constant of a compact Riemannian manifold in terms of its spectral gap and of a lower bound on its curvature. Ledoux also deduced a Gaussian isoperimetric inequality from a logarithmic Sobolev inequality. The argument was extended to the framework of Markov diffusion generators by Bakry and Ledoux [7]. Moreover these authors obtained dimension free constants. The following result is a particular case of [7, Inequality (4.3)]. It allows to turn hypercontractivity properties into isoperimetric inequalities. 
Theorem 40. Let $\mu$ be a probability measure on $\mathbb{R}^{n}$ with $d \mu(x)=e^{-V(x)} d x$ with $V^{\prime \prime} \geq 0$. Let $\left(\mathbf{P}_{t}\right)_{t \geq 0}$ be the corresponding semi-group with generator $\Delta-\nabla V . \nabla$. Then for every $t \geq 0$ and every smooth and bounded function, one has

$$
\|f\|_{2}^{2}-\left\|\mathbf{P}_{t / 2} f\right\|_{2}^{2} \leq \sqrt{2 t}\|f\|_{\infty} \int|\nabla f| d \mu .
$$

In particular (applying this to approximations of characteristic functions) for any Borel set $A \subset \mathbb{R}^{n}$ one has

$$
\mu(A)-\left\|\mathbf{P}_{t / 2} \mathbb{I}_{A}\right\|_{2}^{2} \leq \sqrt{2 t} \mu_{s}(\partial A)
$$

Remark 29. If one only assumes that $V^{\prime \prime} \geq-R \cdot I d$ for $R>0$ then the statement is valid with an additional factor $(2 t R /(1-\exp (-2 t R)))^{1 / 2}$ on the right-hand side. This factor is essentially a constant when $t \leq 1 / R$.

In order to exploit this result we need the following two lemmas.

Lemma 41. Let the measure $\mu$ and the semi-group $\left(\mathbf{P}_{t}\right)_{t \geq 0}$ be as before. Let $\tau$ be a Young function, and assume that for all $f \in \mathbb{L}^{2}(\mu)$ one has $N_{\tau}\left(\mathbf{P}_{t} f\right) \leq C\|f\|_{2}$. Then for every Borel subset $A$ of $\mathbb{R}^{n}$ one has $\left\|\mathbf{P}_{t} \mathbb{I}_{A}\right\|_{2} \leq$ $C \mu(A) \tau^{-1}\left(\frac{\tau(1)}{\mu(A)}\right)$, where $\tau^{-1}$ stands for the reciprocal function of $\tau$.

Proof. Since $\mathbf{P}_{t}$ is symmetric for $\mu$, one gets by duality that $\mathbf{P}_{t}$ maps the dual of $\left(\mathbb{L}_{\tau}(\mu), N_{\tau}\right)$ into $\mathbb{L}^{2}(\mu)$ with norm at most $C$. So for every $A$, $\left\|\mathbf{P}_{t} \mathbb{I}_{A}\right\|_{2} \leq C\left\|\mathbb{1}_{A}\right\|_{\tau^{*}}$. Recall that the latter norm is

$$
\begin{aligned}
\left\|\mathbb{I}_{A}\right\|_{\tau^{*}} & =\sup \left\{\int_{A} g d \mu ; \int \tau(g) d \mu \leq \tau(1)\right\} \\
& =\sup \left\{\int_{A} g d \mu ; \int_{A} \tau(g) d \mu \leq \tau(1)\right\}=\mu(A) \tau^{-1}\left(\frac{\tau(1)}{\mu(A)}\right) .
\end{aligned}
$$

Indeed Jensen inequality yields $\int_{A} \tau(g) \frac{d \mu}{\mu(A)} \geq \tau\left(\int_{A} g \frac{d \mu}{\mu(A)}\right)$, which is tight for $g=\mathbb{1}_{A} \tau^{-1}(\tau(1) / \mu(A))$.

Lemma 42. Let $F: \mathbb{R}^{+} \rightarrow \mathbb{R}$ be a non-decreasing function with $F(1)=0$, and continuous on $[1,+\infty)$. Consider for $q, x \geq 0$, the function $\tau_{q}(x)=$ $x^{2} e^{q F\left(x^{2}\right)}$. Assume there exists constants $c_{1}, c_{2}$ such that for all $x \geq 1$ one has $F(x) \leq c_{1} \log x$ and $F\left(x^{2}\right) \leq c_{2} F(x)$. Then for all $q \in\left[0,1 / c_{1}\right]$ one has

$$
\tau_{q}^{-1}(y) \leq \sqrt{y} e^{-\frac{q}{2 c_{2}} F(y)}, \quad y \geq 1
$$


Proof. Set $\Theta(x)=\exp \left(-q F(x) /\left(2 c_{2}\right)\right)$. Setting $y=\tau_{q}(x), x \geq 1$ the claimed inequality can be rephrased as:

$$
x \leq \tau_{q}(x)^{\frac{1}{2}} \Theta\left(\tau_{q}(x)\right)=x e^{\frac{q}{2} F\left(x^{2}\right)} e^{-\frac{q}{2 c_{2}} F\left(x^{2} \exp \left(q F\left(x^{2}\right)\right)\right)}, \quad x \geq 1 .
$$

This is equivalent to $F\left(x^{2} \exp \left(q F\left(x^{2}\right)\right)\right) \leq c_{2} F\left(x^{2}\right)$. The latter follows from the hypotheses: for $q \leq 1 / c_{1}, F\left(x^{2} \exp \left(q F\left(x^{2}\right)\right)\right) \leq F\left(x^{2+2 q c_{1}}\right) \leq F\left(x^{4}\right) \leq$ $c_{2} F\left(x^{2}\right)$.

Theorem 43. Let $\mu$ be a probability measure on $\mathbb{R}^{n}$ with $d \mu(x)=e^{-V(x)} d x$ and $V^{\prime \prime} \geq 0$. Assume that the corresponding semi-group $\left(\mathbf{P}_{t}\right)_{t \geq 0}$ with generator $\Delta-\nabla V \cdot \nabla$ satisfies for every $t \in[0, T]$ and every function in $\mathbb{L}^{2}\left(\mathbb{R}^{n}, \mu\right)$,

$$
N_{\tau_{k t}}\left(\mathbf{P}_{t} f\right) \leq C\|f\|_{2},
$$

where $k>0, C \geq 1$ and for $q \geq 0, x \in \mathbb{R}, \tau_{q}(x)=x^{2} \exp \left(q F\left(x^{2}\right)\right)$. Here $F:[0, \infty) \rightarrow \mathbb{R}$ is non-decreasing and satisfies $F(1)=0$, and for $x \geq 1$, $F(x) \leq c_{1} \log x, F\left(x^{2}\right) \leq c_{2} F(x)$. Then if $A \subset \mathbb{R}^{n}$ has small measure in the sense that $F(1 / \mu(A)) \geq c_{2} \log \left(2 C^{2}\right) / \min \left(k T, 1 / c_{1}\right)$ one has the following isoperimetric inequality:

$$
\mu_{s}(\partial A) \geq \frac{1}{4}\left(\frac{k}{c_{2} \log \left(2 C^{2}\right)}\right)^{\frac{1}{2}} \mu(A) F\left(\frac{1}{\mu(A)}\right)^{\frac{1}{2}} .
$$

The symmetric inequality holds for large sets: if

$$
F\left(\frac{1}{1-\mu(A)}\right) \geq \frac{c_{2} \log \left(2 C^{2}\right)}{\min \left(k T, 1 / c_{1}\right)}
$$

then

$$
\mu_{s}(\partial A) \geq \frac{1}{4}\left(\frac{k}{c_{2} \log \left(2 C^{2}\right)}\right)^{\frac{1}{2}}(1-\mu(A)) F\left(\frac{1}{1-\mu(A)}\right)^{\frac{1}{2}} .
$$

Proof. We combine the above results and choose an appropriate value of the time parameter. If $t \leq \min \left(2 T, 2 /\left(k c_{1}\right)\right.$ then

$$
\begin{aligned}
\mu_{s}(\partial A) & \geq \frac{\mu(A)-\left\|\mathbf{P}_{t / 2} \mathbb{I}_{A}\right\|_{2}^{2}}{\sqrt{2 t}} \\
& \geq \frac{\mu(A)-\left(C \mu(A) \tau_{k t / 2}^{-1}\left(\frac{1}{\mu(A)}\right)\right)^{2}}{\sqrt{2 t}} \geq \mu(A) \frac{1-C^{2} \exp \left(-\frac{k t}{2 c_{2}} F\left(\frac{1}{\mu(A)}\right)\right)}{\sqrt{2 t}} .
\end{aligned}
$$

At this point we wish to choose $t$ so that $\frac{1}{2}=C^{2} \exp \left(-\frac{k t}{2 c_{2}} F\left(\frac{1}{\mu(A)}\right)\right)$. This is compatible with the

$$
F(1 / \mu(A)) \geq c_{2} \log \left(2 C^{2}\right) / \min \left(k T, 1 / c_{1}\right) .
$$


Under this condition, this value of time yields the claimed isoperimetric inequality for small sets. For large sets note that applying the functional inequality of Theorem 40 to suitable approximations of the characteristic function of $A^{c}$ gives $\sqrt{2 t} \mu_{s}(\partial A) \geq \mu\left(A^{c}\right)-\left\|\mathbf{P}_{t / 2} \mathbb{I}_{A^{c}}\right\|_{2}^{2}$, so the study of small sets apply.

Remark 30. Under the weaker assumption $V^{\prime \prime} \geq-R$ for $R>0$ we have similar results with constants depending on $R$.

Remark 31. Under specific assumptions on $F$ we have shown that $\operatorname{Cap}_{\mu}(A) \geq$ $\mu(A) F(1 / \mu(A))$ for all $A$ implies continuity of the semigroup in the Orlicz scale $\tau_{q}(x)=x^{2} \exp \left(q F\left(x^{2}\right)\right)$, which implies, at least for small sets, $\mu_{s}(\partial A) \geq$ $K \mu(A) \sqrt{F(1 / \mu(A))}$. Note the analogy between these relations and also the inequality

$$
\mu_{s}(\partial A) \geq \operatorname{Cap}_{\mu}^{(1)}(A):=\inf \left\{\int|\nabla f| d \mu ; f \geq \mathbb{1}_{A} \text { and } \mu(f=0) \geq 1 / 2\right\} .
$$

The previous theorem provides a lower bound on the isoperimetric profile for small and large values of the measure only. We deal with the remaining values, away from 0 and 1, by means of Cheeger's inequality. The dimension free version of Buser's inequality for diffusion generator, contained in the work of Bakry and Ledoux allows to derive Cheeger's inequality from Poincaré inequality.

Theorem 44. Let $\mu$ be a probability measure on $\mathbb{R}^{n}$ with $d \mu(x)=e^{-V(x)} d x$ and $V^{\prime \prime} \geq 0$. Assume that the corresponding semi-group $\left(\mathbf{P}_{t}\right)_{t \geq 0}$ with generator $\Delta-\nabla V \cdot \nabla$ satisfies the following Poincaré inequality: for all $f$

$$
\lambda \int(f-\mu(f))^{2} d \mu \leq \int|\nabla f|^{2} d \mu .
$$

Then for every Borel set $A \subset \mathbb{R}^{n}$ one has

$$
\mu_{s}(\partial A) \geq \frac{1-e^{-1}}{\sqrt{2}} \sqrt{\lambda} \mu(A)(1-\mu(A))
$$

The argument is written in the setting of Riemannian manifolds in [40, Theorem 5.2]. We sketch the proof for completeness.

Proof. The spectral gap inequalities classically implies the exponential decay of the norm of $\mathbf{P}_{t}$ on the space of zero mean. Therefore

$$
\begin{aligned}
\left\|\mathbf{P}_{t / 2} \mathbb{I}_{A}\right\|_{2}^{2} & =\left\|\mathbf{P}_{t / 2} \mu(A)\right\|_{2}^{2}+\left\|\mathbf{P}_{t / 2}\left(\mathbb{I}_{A}-\mu(A)\right)\right\|_{2}^{2} \\
& \leq \mu(A)^{2}+e^{-\lambda t}\left\|\mathbb{I}_{A}-\mu(A)\right\|_{2}^{2}=\mu(A)^{2}+e^{-\lambda t} \mu(A)(1-\mu(A)) .
\end{aligned}
$$

By Theorem 40, one has

$$
\sqrt{2 t} \mu_{s}(\partial A) \geq\left(1-e^{-\lambda t}\right) \mu(A)(1-\mu(A)) .
$$

Choosing $t=1 / \lambda$ concludes the proof. 
Finally we apply the previous results to infinite products of the measures:

$$
m_{\alpha}(d x)=\frac{\exp \left(-|x|^{\alpha}\right)}{2 \Gamma(1+1 / \alpha)} d x, \quad x \in \mathbb{R}
$$

For technical reasons, we also consider the measures $\nu_{\alpha}$ defined in section 7 up to the irrelevant constant 2. They also have a log-concave density, but more regular. The isoperimetric function of a symmetric log-concave density on the line (with the usual metric) was calculated by Bobkov [14]. He showed that half-lines have minimal boundary among sets of the same measure. Since the boundary measure of $(-\infty, t]$ is given by the density of the measure at $t$, the isoperimetric profile is easily computed. They are readily compared to the functions

$$
L_{\alpha}(t)=\min (t, 1-t) \log ^{1-\frac{1}{\alpha}}\left(\frac{1}{\min (t, 1-t)}\right) .
$$

We omit the details, some of them are written in [9].

Lemma 45. There are constants $k_{1}, k_{2}$ such that for all $\alpha \in[1,2], t \in[0,1]$ one has

$$
\begin{gathered}
k_{1} L_{\alpha}(t) \leq I_{m_{\alpha}}(t) \leq k_{2} L_{\alpha}(t), \\
k_{1} L_{\alpha}(t) \leq I_{\nu_{\alpha}}(t) \leq k_{2} L_{\alpha}(t) .
\end{gathered}
$$

Our goal is to show the following infinite dimensional isoperimetric inequality.

Theorem 46. There exists a constant $K>0$ such that for all $\alpha \in[1,2]$ and $t \in[0,1]$, one has

$$
I_{\nu_{\alpha}^{\infty}}(t) \geq K L_{\alpha}(t)
$$

Since $I_{\nu_{\alpha}^{\infty}} \leq I_{\nu_{\alpha}} \leq k_{2} L_{\alpha}$, we have, up to a constant, the value of the isoperimetric profile of the infinite product.

Proof. As shown in Corollary 34 of Section 7 the semi-group associated to $\nu_{\alpha}^{\otimes n}$ is Orlicz-hyperbounded. Thus we may apply Theorem 43 with $F=F_{\alpha}$ defined in (7.2) and get an isoperimetric inequality for small and large sets, with constants independent of the dimension $n$. This step requires to check a few properties of the function $F_{\alpha}$. They are established in the following Lemma 47. More precisely there are constants $K_{1}, K_{2}>0$ independent of $\alpha$ and $n$ such that, denoting $\beta(\alpha)=2(1-1 / \alpha)$

$$
I_{\nu_{\alpha}^{\otimes n}}(t) \geq K_{1} \min (t, 1-t)\left[\log ^{\beta(\alpha)}\left(1+\frac{1}{\min (t, 1-t)}\right)-\log ^{\beta(\alpha)}(2)\right]^{\frac{1}{2}}
$$


provided

$$
\left(\log ^{\beta(\alpha)}\left(1+\frac{1}{\min (t, 1-t)}\right)-\log ^{\beta(\alpha)}(2)\right)^{\frac{1}{2}} \geq K_{2} .
$$

We can prove (8.1) in the remaining range as well. Indeed, it is plain that

$$
\sup _{x>0} \nu_{\alpha}([x,+\infty)) \int_{0}^{x} \frac{1}{\rho_{\nu_{\alpha}}} \leq M,
$$

so that the measures $\left(\nu_{\alpha}\right)_{\alpha \in[1,2]}$ satisfy a Poincaré inequality with a uniform constant. The latter inequality has the tensorisation property, so the measures $\nu_{\alpha}^{\otimes n}$ also share a common Poincaré inequality. By Theorem 44, there exists a constant $K_{3}>0$ such that for all $n$, all $\alpha \in[1,2]$ and all $t \in[0,1]$

$$
I_{\nu_{\alpha}^{\otimes n}}(t) \geq K_{3} \min (t, 1-t) .
$$

Since the exponential measure has a spectral gap, the latter argument reproves, with a slightly worse constant, the result of [16]. Now assume that

$$
\log ^{\beta(\alpha)}\left(1+\frac{1}{\min (t, 1-t)}\right)-\log ^{\beta(\alpha)}(2)<K_{2}^{2},
$$

then

$$
\begin{aligned}
I_{\nu_{\alpha}^{\otimes n}}(t) & \geq \frac{K_{3}}{K_{2}} \min (t, 1-t) K_{2} \\
& \geq \frac{K_{3}}{K_{2}} \min (t, 1-t)\left[\log ^{\beta(\alpha)}\left(1+\frac{1}{\min (t, 1-t)}\right)-\log ^{\beta(\alpha)}(2)\right]^{\frac{1}{2}}
\end{aligned}
$$

So Inequality (8.1) is valid for all $t \in[0,1]$ provided one replaces $K_{1}$ by

$$
K_{4}:=\min \left(K_{1}, \frac{K_{3}}{K_{2}}\right) \text {. }
$$

Finally, the uniform Cheeger inequality (8.2), implies that

$$
\frac{1}{K_{3}} I_{\nu_{\alpha}^{\otimes n}}(t) \geq \log ^{\beta(\alpha) / 2}(2) \min (t, 1-t) .
$$

Adding up this relation to

$$
\begin{aligned}
\frac{1}{K_{4}} I_{\nu_{\alpha} n}(t) & \geq \min (t, 1-t)\left[\log ^{\beta(\alpha)}\left(1+\frac{1}{\min (t, 1-t)}\right)-\log ^{\beta(\alpha)}(2)\right]^{\frac{1}{2}} \\
& \geq \min (t, 1-t)\left[\log ^{\beta(\alpha) / 2}\left(1+\frac{1}{\min (t, 1-t)}\right)-\log ^{\beta(\alpha) / 2}(2)\right]
\end{aligned}
$$

yields the claimed inequality. This manipulation was important in order to get a non-trivial inequality when $\alpha$ tends to 1 , i.e. when $\beta(\alpha)$ tends to 0 . 
The following technical result was used in the above proof.

Lemma 47. Let $\beta \in[0,1]$ then for all $x \geq 1$ one has

$$
\begin{aligned}
\log ^{\beta}(1+x)-\log ^{\beta}(2) & \leq \log x \\
\log ^{\beta}\left(1+x^{2}\right)-\log ^{\beta}(2) & \leq 8\left(\log ^{\beta}(1+x)-\log ^{\beta}(2)\right) .
\end{aligned}
$$

Proof. Note that (8.3) is an equality for $x=1$. It is enough to prove the inequality between derivatives, that is

$$
\beta \frac{\log ^{\beta-1}(1+x)}{(1+x)} \leq \frac{1}{x} \quad \text { for } x \geq 1
$$

If $x \geq e-1$ then $\log ^{\beta-1}(1+x) \leq 1$ and the inequality is obvious. If $x<e-1$, then $\log ^{\beta}(1+x) \leq 1$, therefore

$$
\beta \frac{\log ^{\beta-1}(1+x)}{(1+x)} \leq \frac{1}{(1+x) \log (1+x)} \leq \frac{1}{x} .
$$

Next we address (8.4). One easily checks that for $A \geq B \geq 1$ the map

$$
\beta>0 \mapsto \frac{A^{\beta}-1}{B^{\beta}-1}
$$

is non-decreasing. Applying this to

$$
A=\log \left(1+x^{2}\right) / \log (2) \quad \text { and } \quad B=\log (1+x) / \log (2)
$$

shows that it is enough to prove (8.4) for $\beta=1$. Let $x \geq 1$, since $1+x^{2} \leq$ $(1+x)^{2}$ one has

$\log \left(1+x^{2}\right)-\log (2) \leq 2 \log (1+x)-\log (2)=2(\log (1+x)-\log (2))+\log (2)$.

If $x \geq 3$ then $\log (1+x)-\log (2) \geq \log (2)$ and the claimed inequality is proved. For $x \in(1,3]$, we use the fundamental relation of calculus. It provides $t_{1} \in(1,9)$ and $t_{2} \in(1,3)$ with

$$
\begin{aligned}
& \qquad \log \left(1+x^{2}\right)-\log (2)=\left(x^{2}-1\right) \frac{1}{1+t_{1}} \leq 2(x-1) \\
& \text { and } \log (1+x)-\log (2)=(x-1) \frac{1}{1+t_{2}} \geq(x-1) / 4 \text {. }
\end{aligned}
$$

So the ratio is bounded from above by 8 . A smarter choice than 3 would give a better result. 
Remark 32. According to Theorem 39 the conclusion of Theorem 46 is still true with the same $L_{\alpha}$ when replacing $\nu_{\alpha}$ by $\nu_{V}$, provided $V$ is convex and the hypotheses in Theorem 39 are fulfilled.

We conclude the paper with consequences of Theorem 46. The first one is a comparison theorem. It could be stated in a more general framework of metric probability spaces satisfying a smoothness assumption (see e.g. [10]). For simplicity we write it in the setting of Riemannian manifolds where the definition of isoperimetric profile given in the beginning of the section applies.

Theorem 48. Let $(X, d, \mu)$ be a Riemannian manifold, with the geodesic metric, and a probability measure which has a density with respect to the volume. On the product manifold we consider the geodesic distance, which is the $\ell_{2}$ combination of the distances on the factors. There exists a universal constant $K>0$ such that if for some $c>0, \gamma \in\left[0, \frac{1}{2}\right]$ and all $t \in[0,1]$ one has

$$
I_{\mu}(t) \geq c \min (t, 1-t) \log ^{\gamma}\left(\frac{1}{\min (t, 1-t)}\right),
$$

then for all $n \geq 1, t \in[0,1]$ one has

$$
I_{\mu \otimes n}(t) \geq \frac{c}{K} \min (t, 1-t) \log ^{\gamma}\left(\frac{1}{\min (t, 1-t)}\right) .
$$

Remark 33. This provides a scale of infinite dimensional isoperimetric inequalities. Both ends of the scale where previously known. A standard argument based on the central limit theorem shows that if $\mu$ is a measure on $\mathbb{R}$ with second moment then $\inf _{n} I_{\mu \otimes n}$ is dominated by a multiple of the Gaussian isoperimetric function, which is comparable to

$$
\min (t, 1-t) \log ^{1 / 2}\left(\frac{1}{\min (t, 1-t)}\right) \text {. }
$$

On the other hand an argument of Talagrand [52] shows that the weakest possible dimension free concentration result for $\mu$ implies that it has at most exponential tails. The isoperimetric function of the exponential density is $\min (t, 1-t)$. So the above scale covers the whole range of infinite isoperimetric inequalities. Of course finer scales could be obtained from our methods, with more effort.

Remark 34. A similar statement was proved in [11] for the case when the distance on the product space is the $\ell_{\infty}$ combination of the distances on the factors (i.e. the maximum). This case was much easier due to the product structure of balls in the product space. Also, this notion leads to bigger enlargement, and the scale of infinite dimension behavior was larger, the values $\gamma \in[0,1]$ being allowed. 
Proof of Theorem 48. The hypothesis implies that

$$
I_{\mu} \geq \frac{c}{k_{2}} I_{\nu_{\alpha}} \quad \text { for } \alpha=1 /(1-\gamma) \in[1,2]
$$

Theorem 10 in [10] asserts that among measures having the same concave isoperimetric behavior, the even log-concave one minimizes the isoperimetric profile for the product measures, see also [48]. So we have

$$
I_{\mu}^{\otimes n} \geq \frac{c}{k_{2}} I_{\nu_{\alpha}^{\otimes n}}
$$

By the previous results $I_{\nu_{\alpha}^{\otimes n}} \geq K L_{\alpha}$ and the proof is complete.

The second consequence that we wish to put forward deals with the measures

$$
d m_{\alpha}(x)=\frac{\exp \left(-|x|^{\alpha}\right)}{2 \Gamma(1+1 / \alpha)} d x, \quad \alpha \in[1,2]
$$

It shows that among sets of prescribed measure for $m_{\alpha}^{\otimes n}$ in $\mathbb{R}^{n}$, coordinate half-spaces have enlargements of minimal measure, up to a universal factor. The result was known for $\alpha \in 1,2$.

Theorem 49. There exists a universal constant $K$ such that for every $\alpha \in$ $[1,2], n \geq 1$ and every Borel set $A \subset \mathbb{R}^{n}$, if $m_{\alpha}^{\otimes n}(A)=m_{\alpha}((-\infty, t])$ then for $h \geq 0$,

$$
m_{\alpha}^{\otimes n}\left(A+h B_{2}^{n}\right) \geq m_{\alpha}\left(\left(-\infty, t+\frac{h}{K}\right]\right) .
$$

Proof. This fact is proved by integrating the inequality

$$
I_{m_{\alpha}^{\otimes n}} \geq \frac{I_{m_{\alpha}}}{K}
$$

which provides a similar information about boundary measure (this corresponds to infinitesimal enlargements).

This isoperimetric inequality is a consequence of the fact that $I_{m_{\alpha}}$ is comparable to $I_{\nu_{\alpha}}$. The comparison theorem of [10] implies that $I_{m_{\alpha}^{\otimes n}}$ is larger than a universal constant times $I_{\nu_{\alpha}^{\otimes n}} \geq K L_{\alpha} \geq \frac{K}{k_{2}} I_{m_{\alpha}}$.

Acknowledgments. We wish to thank Sergey Bobkov, Thierry Coulhon, Arnaud Guillin, Gérard Kerkyacharian, Michel Ledoux, Laurent Miclo, Yves Raynaud and Bogusław Zegarlinski for fruitful discussions and remarks on the topic of this paper. 


\section{References}

[1] Adams, R. A.: General logarithmic Sobolev inequalities and Orlicz imbeddings. J. Funct. Anal. 34 (1979), 292-303.

[2] Aida, S, Masuda, T. And Shigekawa, I.: Logarithmic Sobolev inequalities and exponential integrability. J. Funct. Anal. 126 (1994), 83-101.

[3] Ané, C., Blachère, S., Chafaї, D., Fougères, P., Gentil, I., Malrieu, F., Roberto, C. and Scheffer, G.: Sur les inégalités de Sobolev logarithmiques. Panoramas et Synthèses 10. Société Mathématique de France, Paris, 2000.

[4] BAKRY, D.: L'hypercontractivité et son utilisation en théorie des semigroupes. Lecture Notes in Math. 1581. Springer, Berlin, 1994.

[5] Bakry, D., Coulhon, T., Ledoux, M. And Saloff-Coste, L..: Sobolev inequalities in disguise. Indiana Univ. Math. J. 44 (1995), 10331074.

[6] BAKRy D. AND Émery, M.: Diffusions hypercontractives. In Séminaire de probabilités, XIX, 1983/84, 177-206. Lecture Notes in Math. 1123. Springer, Berlin, 1985.

[7] BAKry, D. And Ledoux, M.: Lévy-Gromov's isoperimetric inequality for an infinite-dimensional diffusion generator. Invent. Math. 123 (1996), 259-281.

[8] BARThe, F.: Extremal properties of central half-spaces for product measures. J. Funct. Anal. 182 (2001), 81-107.

[9] BARThe, F.: Levels of concentration between exponential and Gaussian. Ann. Fac. Sci. Toulouse Math. (6) 10 (2001), 393-404.

[10] Barthe, F.: Log-concave and spherical models in isoperimetry. Geom. Funct. Anal. 12 (2002), 32-55.

[11] BARThe, F.: Infinite dimensional isoperimetric inequalities in product spaces with the supremum distance. J. Theoret. Probab. 17 (2004), 293308.

[12] Barthe, F. and Roberto, C.: Sobolev inequalities for probability measures on the real line. Studia Math. 159 (2003), 481-497.

[13] Beckner, W.: A generalized Poincaré inequality for Gaussian measures. Proc. Amer. Math. Soc. 105 (1989), 397-400.

[14] Bobkov, S.: Extremal properties of half-spaces for log-concave distributions. Ann. Probab. 24 (1996), 35-48.

[15] Bobkov, S. G. And Götze, F.: Exponential integrability and transportation cost related to logarithmic Sobolev inequalities. J. Funct. Anal. 163 (1999), 1-28.

[16] Bobkov, S. G. ANd Houdré, C.: Isoperimetric constants for product probability measures. Ann. Probab. 25 (1997), 184-205.

[17] Bobkov, S. G. And Houdré, C.: Some connections between isoperimetric and Sobolev-type inequalities. Mem. Amer. Math. Soc. 129 (1997), no. 616. 
[18] Bobkov, S. G. And Ledoux, M.: Poincaré's inequalities and Talagrand's concentration phenomenon for the exponential distribution. Probab. Theory Related Fields 107 (1997), 383-400.

[19] Bobkov, S. G. and Zegarlinski, B.: Entropy bounds and isoperimetry. Mem. Amer. Math. Soc. 176 (2005), no. 829.

[20] Boucheron, S., Bousquet, O., Lugosi, G. and Massart, P.: Moment inequalities for functions of independent random variables. Ann. Probab. 33 (2005), 514-560.

[21] Cattiaux, P.: Hypercontractivity for perturbed diffusion semigroups. Ann. Fac. Sci. Toulouse Math. (6) 14 (2005), 609-628.

[22] Cattiaux, P.: A pathwise approach of some classical inequalities. Potential Anal. 20 (2004), 361-394.

[23] Cattiaux, P. And Guillin, A.: On quadratic transportation cost inequalities. J. Math. Pures Appl. 86 (2006), 342-361.

[24] Cattiaux, P. and LÉonard, C.: Minimization of the Kullback information for general Markov processes. In Séminaire de Probabilités XXX, 288-311. Lect. Notes in Math. 1626. Springer, Berlin, 1996.

[25] Chafaї, D.: Entropies, convexity, and functional inequalities: on $\Phi$-entropies and $\Phi$-Sobolev inequalities. J. Math. Kyoto Univ. 44 (2004), 325-363.

[26] Coulhon, T., Grigor'yan, A. and Levin, D.: On isoperimetric profiles of product spaces. Comm. Anal. Geom., 11 (2003), 85-120.

[27] Davies, E. B.: Heat kernels and spectral theory. Cambridge Tracts in Mathematics 92. Cambridge University Press, 1989.

[28] Davies, E. B. And Simon, B.: Ultracontractivity and the heat kernel for Schrödinger operators and Dirichlet Laplacians. J. Funct. Anal. 59 (1984), 335-395.

[29] Gentil, I., Guillin, A. And Miclo, L.: Modified logarithmic Sobolev inequalities and transportation inequalities. Probab. Theory Related Fields 133 (2005), 409-436.

[30] Gong, F. Z. And Wang, F. Y.: Functional inequalities for uniformly integrable semigroups and application to essential spectrums. Forum Math. 14 (2002), 293-313.

[31] Gross, L.: Logarithmic Sobolev inequalities. Amer. J. Math. 97 (1975), 1061-1083.

[32] Gross, L.: Logarithmic Sobolev inequalities and contractivity properties of semigroups. In Dirichlet forms (Varenna, 1992), 54-88. Lecture Notes in Math. 1563. Springer, Berlin, 1993.

[33] Guionnet, A. and Zegarlinski, B.: Lectures on logarithmic Sobolev inequalities. In Séminaire de Probabilités XXXVI, 1-134. Lecture Notes in Math. 1801. Springer, Berlin, 2003.

[34] Høegh-Krohn, A. And Simon, B.: Hypercontractive semigroups and two dimensional self-coupled Bose fields. J. Funct. Anal. 9 (1972), 121-180. 
[35] Kavian, O., Kerkyacharian, G. and Roynette, B.: Quelques remarques sur l'ultracontractivité. J. Funct. Anal. 111 (1993), 155-196.

[36] Latala, R. and Oleszkiewicz, K.: Between Sobolev and Poincaré. In Geometric aspects of functional analysis, 147-168. Lecture Notes in Math. 1745. Springer, Berlin, 2000.

[37] Ledoux, M.: A simple analytic proof of an inequality by P. Buser. Proc. Amer. Math. Soc. 121 (1994), 951-959.

[38] Ledoux, M.. Concentration of measure and logarithmic Sobolev inequalities. In Séminaire de Probabilités XXXIII, 120-216. Lecture Notes in Math. 1709. Springer, Berlin, 1999.

[39] Ledoux, M.: The concentration of measure phenomenon. Mathematical Surveys and Monographs 89. American Mathematical Society, Providence, RI, 2001.

[40] Ledoux, M.: Spectral gap, logarithmic Sobolev constant and geometric bounds. In Surveys in differential geometry. Vol. IX, 219-240. Int. Press, Somerville, MA, 2004.

[41] Maurey, B.: Some deviation inequalities. Geom. Funct. Anal. 1 (1991), $188-197$.

[42] Maz'JA, V. G.: Sobolev spaces. Springer Series in Soviet Mathematics. Springer-Verlag, Berlin, 1985.

[43] Miclo, L. And Roberto, C.: Inégalités de Hardy et de Sobolev logarithmiques. Thèse de Doctorat de $\mathrm{C}$. Roberto, chapter 3. Université Paul Sabatier, 2001.

[44] Muckenhoupt, B.: Hardy's inequality with weights. Studia Math. 44 (1972), 31-38.

[45] Nelson, E.: The free Markov field. J. Funct. Anal. 12 (1973), 211-227.

[46] RaO, M. M. And Ren, Z. D.: Theory of Orlicz spaces. Monographs and Textbooks in Pure and Applied Mathematics 146. Marcel Dekker, Inc., New York, 1991.

[47] Röckner, M. And Wang, F. Y.: Weak Poincaré inequalities and $L^{2}$-convergence rates of Markov semigroups. J. Funct. Anal. 185 (2001), 564-603.

[48] Ros, A.: The isoperimetric problem. In Global theory of minimal surfaces, 175-209, Clay Math. Proc. 2. Amer. Math. Soc., Providence, RI, 2005.

[49] Rosen, J.: Sobolev inequalities for weight spaces and supercontractivity. Trans. Amer. Math. Soc. 222 (1976), 367-376.

[50] Rothaus, O. S.: Analytic inequalities, isoperimetric inequalities and logarithmic Sobolev inequalities. J. Funct. Anal. 64 (1985), 296-313.

[51] Royer, G.: Une initiation aux inégalités de Sobolev logarithmiques. Cours Spécialisés 5. Société Mathématique de France, Paris, 1999.

[52] Talagrand, M.: A new isoperimetric inequality and the concentration of measure phenomenon. In Geometric aspects of functional analysis (198990), 94-124. Lecture Notes in Math. 1469. Springer, Berlin, 1991. 
[53] Talagrand, M.: The supremum of some canonical processes. Amer. J. Math. 116 (1994), 283-325.

[54] Talagrand, M.: Concentration of measure and isoperimetric inequalities in product spaces. Inst. Hautes Études Sci. Publ. Math. 81 (1995), 73-205.

[55] Talagrand, M.: Transportation cost for Gaussian and other product measures. Geom. Funct. Anal. 6 (1996), 587-600.

[56] WANG, F. Y.: A generalization of Poincaré and log-Sobolev inequalities. Potential Anal. 22 (2005), no. 1, 1-15.

[57] Wang, F. Y.: Functional inequalities for empty essential spectrum. J. Funct. Anal. 170 (2000), 219-245.

[58] Wang, F. Y.: Probability distance inequalities on Riemannian manifolds and path spaces. J. Funct. Anal. 206 (2004), 167-190.

[59] Zegarlinski, B.: Entropy bounds for Gibbs measures with non-Gaussian tails. J. Funct. Anal. 187 (2001), 368-395.

Recibido: 4 de enero de 2005

Franck Barthe

Institut de Mathématiques Université Paul Sabatier 31062 Toulouse Cedex 09, France barthe@math.ups-tlse.fr

Patrick Cattiaux Ecole Polytechnique, CMAP route de Saclay F-91128 Palaiseau Cedex, France and

Université de Paris X Nanterre

MODALX, UFR SEGMI 200 avenue de la République F-92001 Nanterre Cedex, France cattiaux@cmapx.polytechnique.fr

Cyril Roberto Université de Marne-la-Vallée et de Paris XII Val-de-Marne LAMA UMR 8050 5 boulevard Descartes 77454 Marne-la-Vallée Cedex 2, France cyril.roberto@univ-mlv.fr 